DIGITALCOMMONS

— @WAYNESTATE -
Journal of Modern Applied Statistical Methods

Volume 9 | Issue 2

$11-1-2010$

\title{
Use of Two Variables Having Common Mean to Improve the Bar-Lev, Bobovitch and Boukai Randomized Response Model
}

Oluseun Odumade

Educational Testing Service, Princeton, New Jersey, oluseunodumade@yahoo.com

Sarjinder Singh

Texas A\&M University, sarjinder@yahoo.com

Follow this and additional works at: http://digitalcommons.wayne.edu/jmasm

Part of the Applied Statistics Commons, Social and Behavioral Sciences Commons, and the Statistical Theory Commons

\section{Recommended Citation}

Odumade, Oluseun and Singh, Sarjinder (2010) "Use of Two Variables Having Common Mean to Improve the Bar-Lev, Bobovitch and Boukai Randomized Response Model," Journal of Modern Applied Statistical Methods: Vol. 9 : Iss. 2 , Article 10. DOI: $10.22237 /$ jmasm/1288584540

Available at: http://digitalcommons.wayne.edu/jmasm/vol9/iss2/10 


\section{Use of Two Variables Having Common Mean to Improve the Bar-Lev, Bobovitch and Boukai Randomized Response Model}

\author{
Oluseun Odumade \\ Educational Testing Service, \\ Princeton, New Jersey
}

\author{
Sarjinder Singh \\ Texas A\&M University, \\ Kingsville
}

A new method to improve the randomized response model due to Bar-Lev, Bobovitch and Boukai (2004) is suggested. It has been observed that if two sensitive (or non sensitive) variables exist that are related to the main study sensitive variable, then those variables could be used to construct ratio type adjustments to the usual estimator of the population mean of a sensitive variable due to Bar-Lev, Bobovitch and Boukai (2004).The relative efficiency of the proposed estimators is studied with respect to the Bar-Lev, Bobovitch and Boukai (2004) models under different situations.

Key words: Randomized response sampling, estimation of population mean, sensitive quantitative variable.

\section{Introduction}

The problem of estimating the population total of a sensitive quantitative variable is well known in survey sampling. Warner (1965) was the first to suggest a method to estimate the proportion of sensitive characters (e.g., induced abortions, drugs used) via use of a randomization device such as a deck of cards or a spinner such that respondents' privacy would be protected (Tracy and Mangat (1996) presented a rich description of the literature). Mangat and Singh (1990) proposed a two-stage randomized response model. Leysieffer and Warner (1976) and Lanke (1975, 1976) studied different randomized response procedures at equal levels of protection of the respondents; later Nayak (1994), Bhargava (1996), Zou (1997), Bhargava and Singh (2001, 2002) and Moors (1997) found that the Mangat and Singh (1990) and Warner (1965) models remain equally efficient at equivalent protection; however, this result is not true for all

Oluseun Odumade is an Associate Psychometric Analyst. Email: oluseunodumade@yahoo.com. Sarjinder Singh is an Assistant Professor in the Department of Mathematics. Email: sarjinder@yahoo.com. randomized response models (Bhargava, 1996; Bhargava \& Singh, 2002). Singh (2003) shows that the Mangat (1994) model remains more efficient than the Warner (1965) model at equal protection: note that the Mangat (1994) model is a special case of the Kuk (1990) model, which is further improved and studied by Gjestvang and Singh (2006). A two stage model developed by Mangat and Singh (1990) was studied by both Kim and Elam (2005) and Kim and Warde (2005). Eichorn and Hayre (1983) suggested a multiplicative model to collect information on sensitive quantitative variables such as, income, tax evasion or amounts of drugs used; this model was further studied by Arnab (1995, 1996). According to Eichorn and Hayre (1983), each respondent in the sample is requested to report a scrambled response $Z_{i}=S Y_{i}$, where $Y_{i}$ is the real value of the sensitive quantitative variable, and $S$ is the scrambling variable whose distribution is assumed to be known. Thus, $E_{R}(S)=\theta$ and $V_{R}(S)=\gamma^{2}$ are assumed to be known and positive, therefore, an estimator of the population mean $\bar{Y}=N^{-1} \sum_{i \in \Omega} Y_{i}$ under simple random with replacement (SRSWR) sampling is given by:

$$
\bar{y}_{E H}=\frac{1}{n} \sum_{i=1}^{n} \frac{Z_{i}}{\theta}
$$




\section{ODUMADE \& SINGH}

with variance

$$
V\left(\bar{y}_{\mathrm{EH}}\right)=\frac{1}{n} \sigma_{y}^{2}+\frac{1}{n} C_{\gamma}^{2} \bar{Y}^{2}\left(1+C_{y}^{2}\right)
$$

where

$$
C_{\gamma}^{2}=\gamma^{2} / \theta^{2}, \bar{Y}=Y / N
$$

and

$$
C_{y}=\sigma_{y} / \bar{Y}
$$

In a randomized response model recently developed by Bar-Lev, Bobovitch and Boukai (2004) (hereafter referred to as the BBB model), the distribution of the responses is given by:

$$
Z_{i}=\left\{\begin{array}{l}
Y_{i} S \text { with probability }(1-p) \\
Y_{i} \quad \text { with probability } p
\end{array}\right.
$$

Thus, each respondent is requested to rotate a spinner unobserved by the interviewer, if the spinner stops in the shaded area, the respondent is requested to report the real response on the sensitive variable, $Y_{i}$; if the spinner stops in the non-shaded area, the respondent is requested to report the scrambled response, $Y_{i} S$, where $S$ is any scrambling variable with a known distribution. Assume that $E(S)=\theta$ and $V(S)=\gamma^{2}$ are known. Let $p$ be the proportion

Figure 1: BBB Randomized Response Device

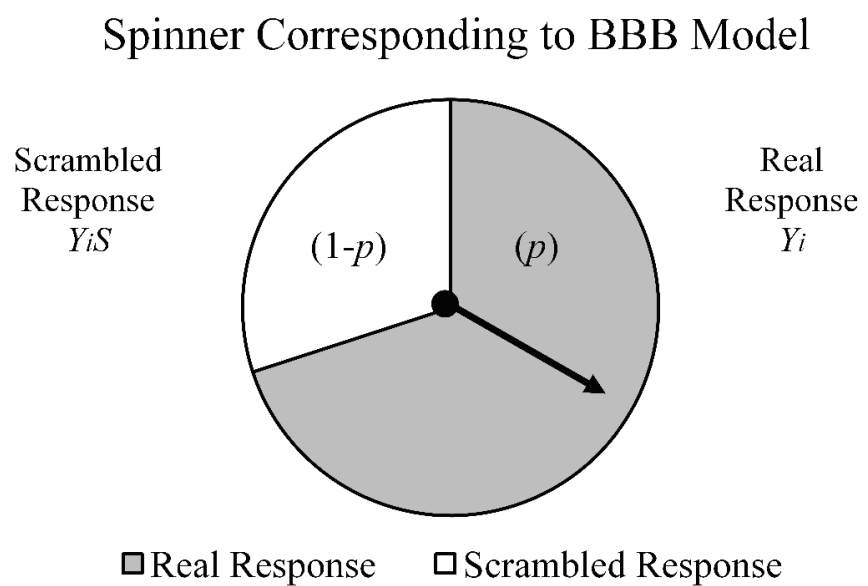

of the shaded area of the spinner and $(1-p)$ be the non-shaded area of the spinner as shown in Figure 1.

An unbiased estimator of population mean $\bar{Y}$ is given by:

$$
\bar{y}_{\mathrm{BBB}}=\frac{1}{n\{(1-p) \theta+p\}} \sum_{i=1}^{n} Z_{i}
$$

with variance under SRSWR sampling given by:

$$
V\left[\bar{y}_{\mathrm{BBB}}\right]=\frac{\bar{Y}^{2}}{n}\left[C_{y}^{2}+\left(1+C_{y}^{2}\right) C_{p}^{2}\right]
$$

where

$$
C_{p}^{2}=\frac{(1-p) \theta^{2}\left(1+C_{\gamma}^{2}\right)+p}{[(1-p) \theta+p]^{2}}-1 .
$$

Notations

Let $\bar{X}_{1 i}=\bar{X}_{2 i}=\bar{X}$ be two auxiliary sensitive variables that have a common mean (Tripathi \& Chaubey, 1992), and let $Y_{i}$ be the sensitive variable under study whose mean is to be estimated. Consider a simple random sample of $n$ respondents selected with replacement (SRSWR), where each respondent selected in the sample is requested to rotate three spinners (see Figure 2).

The first spinner is used to collect scrambled response $Z_{i}$ on the real study variable $Y_{i}$ with the distribution of responses as:

$$
Z_{i}=\left[\begin{array}{l}
Y_{i} \quad \text { with probability } p \\
Y_{i} S \quad \text { with probability }(1-p)
\end{array}\right.
$$

where the value of $p$ is assumed to be known. The second spinner is used to collect scrambled response $Z_{1 i}$ on the first auxiliary sensitive variable $X_{1 i}$ with the distribution of responses as:

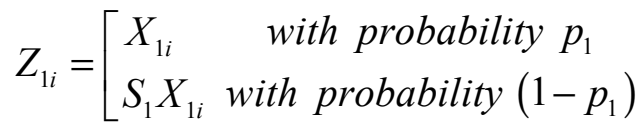


Figure 2: Three Spinners

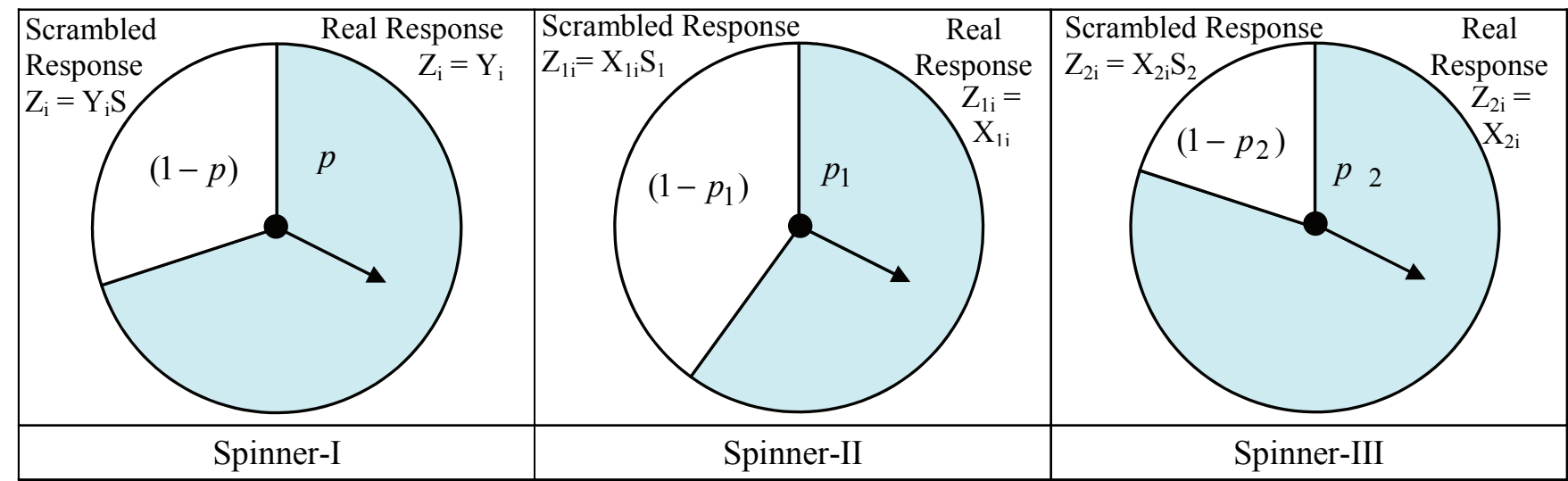

where the value of $p_{1}$ is assumed to be known. The third spinner is used to collect scrambled response $Z_{2 i}$ on the second auxiliary sensitive variable $X_{2 i}$ with the distribution of responses as:

$$
Z_{2 i}=\left[\begin{array}{l}
X_{2 i} \quad \text { with probability } p_{2} \\
S_{2} X_{2 i} \text { with probability }\left(1-p_{2}\right)
\end{array}\right.
$$

where the value of $p_{2}$ is assumed to be known.

Assume that the sample means of the scrambled responses obtained from the respondents in the sample $Z_{i}, Z_{1 i}$ and $Z_{2 i}$ are given by:

$$
\begin{gathered}
\bar{y}^{*}=\frac{1}{p+(1-p) \theta}\left(\frac{1}{n} \sum_{i=1}^{n} Z_{i}\right), \\
\bar{x}_{1}^{*}=\frac{1}{p_{1}+\left(1-p_{1}\right) \theta_{1}}\left(\frac{1}{n} \sum_{i=1}^{n} Z_{1 i}\right),
\end{gathered}
$$

and

$$
\bar{x}_{2}^{*}=\frac{1}{p_{2}+\left(1-p_{2}\right) \theta_{2}}\left(\frac{1}{n} \sum_{i=1}^{n} Z_{2 i}\right) .
$$

Defining $\in=\frac{\bar{y}^{*}}{\bar{Y}}-1, \delta=\frac{\bar{x}_{1}^{*}}{\bar{X}}-1, \eta=\frac{\bar{x}_{2}^{*}}{\bar{X}}-1$, such that $E(\in)=E(\delta)=E(\eta)=0$, it can be shown that

$$
\begin{gathered}
E\left(\in^{2}\right)=\frac{1}{\bar{Y}^{2}} V\left(\bar{y}^{*}\right)=\frac{1}{n}\left[C_{y}^{2}+\left(1+C_{y}^{2}\right) C_{p}^{2}\right], \\
E\left(\delta^{2}\right)=\frac{1}{n}\left[C_{x_{1}}^{2}+\left(1+C_{x_{1}}^{2}\right) C_{p_{1}}^{2}\right] \\
E\left(\eta^{2}\right)=\frac{1}{n}\left[C_{x_{2}}^{2}+\left(1+C_{x_{2}}^{2}\right) C_{p_{2}}^{2}\right]
\end{gathered}
$$

$E(\in \delta)=\frac{1}{n[p+(1-p) \theta]\left[p_{1}+\left(1-p_{1}\right) \theta_{1}\right]} \rho_{Y X_{1}} C_{y} C_{x_{1}}$,

$E(\in \eta)=\frac{1}{n[p+(1-p) \theta]\left[p_{2}+\left(1-p_{2}\right) \theta_{2}\right]} \rho_{Y X_{2}} C_{y} C_{x_{2}}$

and

$$
E(\delta \eta)=\frac{1}{n\left[p_{1}+\left(1-p_{1}\right) \theta_{1}\right]\left[p_{2}+\left(1-p_{2}\right) \theta_{2}\right]} \rho_{X_{1} X_{2}} C_{x_{1}} C_{x_{2}}
$$

where

$$
\begin{gathered}
E\left(Z_{i}\right)=p Y_{i}+(1-p) Y_{i} \theta=[p+(1-p) \theta] Y_{i}, \\
V\left(X_{1 i}\right)=\sigma_{X_{1}}^{2}, V\left(X_{2 i}\right)=\sigma_{X_{2}}^{2}, \\
C_{p}^{2}=\frac{(1-p) \theta^{2}\left(1+C_{\gamma}^{2}\right)+p}{((1-p) \theta+p)^{2}}-1,
\end{gathered}
$$




\section{ODUMADE \& SINGH}

$$
\begin{aligned}
C_{p_{1}}^{2} & =\frac{\left(1-p_{1}\right) \theta_{1}^{2}\left(1+C_{\gamma_{1}}^{2}\right)+p_{1}}{\left(\left(1-p_{1}\right) \theta_{1}+p_{1}\right)^{2}}-1, \\
C_{p_{2}}^{2} & =\frac{\left(1-p_{2}\right) \theta_{2}^{2}\left(1+C_{\gamma_{2}}^{2}\right)+p_{2}}{\left(\left(1-p_{2}\right) \theta_{2}+p_{2}\right)^{2}}-1,
\end{aligned}
$$

and

$$
\begin{aligned}
& \operatorname{Cov}\left(\bar{y}^{*}, \bar{x}_{1}^{*}\right) \\
& =E_{1}\left[C_{2}\left(\bar{y}^{*}, \bar{x}_{1}\right)\right]+C_{1}\left[E_{2}\left(\bar{y}^{*}\right), E_{1}\left(\bar{x}_{1}^{*}\right)\right] \\
& =E_{1}\left[E_{2}\left(\bar{y}^{*} \cdot \bar{x}_{1}\right)-E_{2}\left(\bar{y}^{*}\right) E_{2}\left(\bar{x}_{1}\right)\right] \\
& +C_{1}\left[E_{2}\left(\bar{y}^{*}\right), E_{1}(\bar{x})\right] \\
& =E_{1}\left[\begin{array}{l}
E_{2}\left\{\left(\frac{1}{n^{2}} \sum_{i=1}^{n} Z_{i}\right)\left(\sum_{i=1}^{n} Z_{1 i}\right)\right\} \\
-E_{2}\left(\frac{1}{n} \sum_{i=1}^{n} Z_{i}\right) E_{2}\left(\frac{1}{n} \sum_{i=1}^{n} Z_{1 i}\right)
\end{array}\right] \\
& =0+C_{1}\left[\begin{array}{l}
\frac{1}{p+(1-p) \theta} \frac{1}{n} \sum_{i=1}^{n} y_{i}, \\
\frac{1}{p_{1}+\left(1-p_{1}\right) \theta_{1}} \frac{1}{n} \sum_{i=1}^{n} x_{1 i}
\end{array}\right] \\
& =\frac{\bar{Y} \bar{X}_{1}}{n[p+(1-p) \theta]\left[p_{1}+\left(1-p_{1}\right) \theta_{1}\right]} \rho_{X_{1} Y_{1}} C_{y} C_{x_{1}}
\end{aligned}
$$

Proposed Ratio-Type Estimator

A ratio estimator is defined as:

$$
\bar{y}_{\text {Ratio }}^{*}=\bar{y}^{*}\left(\frac{\bar{x}_{1}^{*}}{\bar{x}_{2}^{*}}\right) \text {. }
$$

Note that:

$$
\begin{aligned}
& \bar{y}^{*}=\bar{Y}(1+\epsilon), \\
& \bar{x}_{1}^{*}=\bar{X}(1+\delta)
\end{aligned}
$$

and

$$
\bar{x}_{2}^{*}=\bar{X}(1+\eta)
$$

thus, the ratio estimator (3.1) can be written in terms of $\in, \delta$ and $\eta$ as:

$$
\begin{aligned}
\bar{y}_{\text {Ratio }}^{*} & =\bar{Y}(1+\epsilon) \frac{\bar{X}(1+\delta)}{\bar{X}(1+\eta)} \\
& =\bar{Y}(1+\epsilon)(1+\delta)(1+\eta)^{-1} \\
& =\bar{Y}[1+\epsilon+\delta+\epsilon \delta]\left[1-\eta+\eta^{2}+\ldots .\right] \\
& =\bar{Y}\left[1+\epsilon+\delta-\eta+\eta^{2}+\epsilon \delta-\epsilon \eta-\delta \eta+\ldots\right]
\end{aligned}
$$

From this, the following theorems result.

Theorem 3.1

The bias in the proposed ratio estimator

$$
\bar{y}_{\text {Ratio }}^{*} \text { is given by }
$$

$$
\begin{aligned}
& B\left(\bar{y}_{\text {Ratio }}^{*}\right)= \\
& \frac{\bar{Y}}{n}\left[C_{x_{2}}^{2}+\left(1+C_{x_{2}}^{2}\right) C_{p_{2}}^{2}\right. \\
& +\frac{1}{[p+(1-p) \theta]\left[p_{1}+\left(1-p_{1}\right) \theta_{1}\right]} \rho_{Y X_{1}} C_{y} C_{x_{1}} \\
& -\frac{1}{[p+(1-p) \theta]\left[p_{2}+\left(1-p_{2}\right) \theta_{2}\right]} \rho_{Y X_{2}} C_{y} C_{x_{2}} \\
& \left.-\frac{1}{\left[p_{1}+\left(1-p_{1}\right) \theta_{1}\right]\left[p_{2}+\left(1-p_{2}\right) \theta_{2}\right]} \rho_{X_{1} X_{2}} C_{x_{1}} C_{x_{2}}\right]
\end{aligned}
$$

Theorem 3.1: Proof

Taking the expected value on both sides of (3.2) results in:

$$
E\left(\bar{y}_{\text {Ratio }}^{*}\right)=\bar{Y}\left[\begin{array}{l}
1+0+0-0+E\left(\eta^{2}\right)+E(\in \delta) \\
-E(\in \eta)-E(\delta \eta)+\ldots
\end{array}\right]
$$

Thus the bias in the proposed ratio estimator $\bar{y}_{\text {Ratio }}^{*}$ is given by: 


$$
\begin{aligned}
& B\left(\bar{y}_{\text {Ratio }}^{*}\right) \\
& =E\left(\bar{y}_{\text {Ratio }}^{*}\right)-\bar{Y} \\
& =\bar{Y}\left[E\left(\eta^{2}\right)+E(\in \delta)-E(\in \eta)-E(\delta \eta)\right] \\
& =\frac{\bar{Y}}{n}\left[C_{x_{2}}^{2}+\left(1+C_{x_{2}}^{2}\right) C_{p_{2}}^{2}\right. \\
& \quad+\frac{1}{[p+(1-p) \theta]\left[p_{1}+\left(1-p_{1}\right) \theta_{1}\right]} \rho_{Y X_{1}} C_{y} C_{x_{1}} \\
& \quad-\frac{1}{[p+(1-p) \theta]\left[p_{2}+\left(1-p_{2}\right) \theta_{2}\right]} \rho_{Y X_{2}} C_{y} C_{x_{2}} \\
& \left.\quad-\frac{1}{\left[p_{1}+\left(1-p_{1}\right) \theta_{1}\right]\left[p_{2}+\left(1-p_{2}\right) \theta_{2}\right]} \rho_{X_{1} X_{2}} C_{x_{1}} C_{x_{2}}\right]
\end{aligned}
$$

Thus, Theorem 3.1 is proved.

Theorem 3.2

The mean squared error of the proposed ratio estimator $\bar{y}_{\text {Ratio }}^{*}$ is given by

$$
\begin{aligned}
& \operatorname{MSE}\left(\bar{y}_{\text {Ratio }}^{*}\right)= \\
& \frac{\bar{Y}^{2}}{n}\left[C_{y}^{2}+\left(1+C_{y}^{2}\right) C_{p}^{2}+C_{x_{1}}^{2}\right. \\
&+\left(1+C_{x_{1}}^{2}\right) C_{p_{1}}^{2}+C_{x_{2}}^{2}+\left(1+C_{x_{2}}^{2}\right) C_{p_{2}}^{2} \\
&+\frac{2 \rho_{Y X_{1}} C_{y} C_{x_{1}}}{[p+(1-p) \theta]\left[p_{1}+\left(1-p_{1}\right) \theta_{1}\right]} \\
&-\frac{2 \rho_{Y X_{2}} C_{y} C_{x_{2}}}{[p+(1-p) \theta]\left[p_{2}+\left(1-p_{2}\right) \theta_{2}\right]} \\
&\left.-\frac{2 \rho_{X_{1} X_{2}} C_{x_{1}} C_{x_{2}}}{\left[p_{1}+\left(1-p_{1}\right) \theta_{1}\right]\left[p_{2}+\left(1-p_{2}\right) \theta_{2}\right]}\right]
\end{aligned}
$$

Theorem 3.2: Proof

The mean squared error of the ratio estimator $\bar{y}_{\text {Ratio }}^{*}$ is given by

$$
\begin{aligned}
& \operatorname{MSE}\left(\bar{y}_{\text {Ratio }}^{*}\right) \\
& =E\left[\bar{y}_{\text {Ratio }}^{*}-\bar{Y}\right]^{2} \\
& =\bar{Y}^{2} E[\in+\delta-\eta]^{2} \\
& =\bar{Y}^{2} E\left[\begin{array}{l}
\in^{2}+\delta^{2}+\eta^{2} \\
+2 \in \delta-2 \in \eta-2 \delta \eta
\end{array}\right] \\
& =\frac{\bar{Y}^{2}}{n}\left[C_{y}^{2}+\left(1+C_{y}^{2}\right) C_{p}^{2}+C_{x_{1}}^{2}\right. \\
& +\left(1+C_{x_{1}}^{2}\right) C_{p_{1}}^{2}+C_{x_{2}}^{2}+\left(1+C_{x_{2}}^{2}\right) C_{p_{2}}^{2} \\
& +\frac{2 \rho_{Y X_{1}} C_{y} C_{x_{1}}}{[p+(1-p) \theta]\left[p_{1}+\left(1-p_{1}\right) \theta_{1}\right]} \\
& -\frac{2 \rho_{Y X_{2}} C_{y} C_{x_{2}}}{[p+(1-p) \theta]\left[p_{2}+\left(1-p_{2}\right) \theta_{2}\right]} \\
& \left.-\frac{2 \rho_{X_{1} X_{2}} C_{x_{1}} C_{x_{2}}}{\left[p_{1}+\left(1-p_{1}\right) \theta_{1}\right]\left[p_{2}+\left(1-p_{2}\right) \theta_{2}\right]}\right]
\end{aligned}
$$

thus proving Theorem 3.2.

Efficiency of the Proposed Ratio Estimator

The proposed ratio estimator $\bar{y}_{\text {Ratio }}^{*}$ will be more efficient than the BBB model if

$$
V\left(\bar{y}_{\text {Ratio }}^{*}\right)<V\left(\bar{y}_{B B B}\right)
$$

Using (1.5) and (3.4), results in:

$$
C_{x_{1}}^{2}+\left(1+C_{x_{1}}^{2}\right) C_{p_{1}}^{2}+C_{x_{2}}^{2}+\left(1+C_{x_{2}}^{2}\right) C_{p_{2}}^{2}
$$

being less than $(<)$

$$
\begin{aligned}
& \frac{2 \rho_{Y X_{2}} C_{y} C_{x_{2}}}{[p+(1-p) \theta]\left[p_{2}+\left(1-p_{2}\right) \theta_{2}\right]} \\
& +\frac{2 \rho_{X_{1} X_{2}} C_{x_{1}} C_{x_{2}}}{\left[p_{1}+\left(1-p_{1}\right) \theta_{1}\right]\left[p_{2}+\left(1-p_{2}\right) \theta_{2}\right]} \\
& -\frac{2 \rho_{Y X_{1}} C_{y} C_{x_{1}}}{[p+(1-p) \theta]\left[p_{1}+\left(1-p_{1}\right) \theta_{1}\right]}
\end{aligned}
$$




\section{ODUMADE \& SINGH}

In order to see the magnitude of the proposed ratio estimator $\bar{y}_{\text {Ratio }}^{*}$ with respect to the BBB model the percent relative efficiency is computed as:

$$
\operatorname{RE}\left(\bar{y}_{\mathrm{BBB}}, \bar{y}_{\mathrm{Ratio}}^{*}\right)=\frac{V\left(\bar{y}_{\mathrm{BBB}}\right)}{\operatorname{MSE}\left(\overline{\mathrm{y}}_{\text {Ratio }}^{*}\right)} \times 100 \%
$$

The relative efficiency of the ratio estimator depends on a few parameters such as $P, P_{1}, P_{2}$, $C_{y}, C_{x_{1}}, C_{x_{2}}, C_{\gamma}, C_{\gamma_{1}}, C_{\gamma_{2}}, \theta, \theta_{1}$ and $\theta_{2}$. The percent relative efficiency (RE) is free from the sample size $n$ and main parameter of interest $\bar{Y}$. Fortran programs were developed in order to find the values of the parameters $C_{y}$, $C_{x_{1}}, C_{x_{2}}, C_{\gamma}, C_{\gamma_{1}}, C_{\gamma_{2}}, \rho_{y x_{1}}, \rho_{y x_{2}}, \rho_{x_{1} x_{2}}$, $\theta, \theta_{1}$ and $\theta_{2}$ by holding $P, P_{1}$, and $P_{2}$ equal to 0.7 such that the percent RE remains greater than $200 \%$. (Detailed results are shown in Table 3.1 in the Appendix.)

Values of $C_{y}, C_{x_{1}}, C_{x_{2}}, C_{\gamma}, C_{\gamma_{1}}$, and $C_{\gamma_{2}}$ were changed between 0.1 and 0.5 with a step of 0.2 , and the values $\theta, \theta_{1}$ and $\theta_{2}$ were changed between 0 and 1 with a step of 0.5 . It was observed that selecting larger values for $\theta, \theta_{1}$ and $\theta_{2}$ may lead to inefficient results, thus the choice of these values is critical when using the proposed ratio method in practice.

Table 3.2: Descriptive Statistics of the Percent Relative Efficiency

\begin{tabular}{|c|c|}
\hline Mean & 289.9 \\
\hline Standard Error & 2.9 \\
\hline Median & 270.0 \\
\hline Standard Deviation & 77.4 \\
\hline Sample Variance & 5994.6 \\
\hline Kurtosis & -0.1 \\
\hline Skewness & 0.9 \\
\hline Range & 298.9 \\
\hline Minimum & 200.9 \\
\hline Maximum & 499.8 \\
\hline Count & 724 \\
\hline
\end{tabular}

The values of $\rho_{y x_{2}}$ and $\rho_{x_{1} x_{2}}$ were changed between 0.1 and 0.9 with a step of 0.2 , and $\rho_{y x_{1}}$ was changed between -0.9 to +0.9 with a step of 0.2 . The average percent relative efficiency was $289.9 \%$ with a standard deviation of 77.4 , median of $270.0 \%$, minimum of $200.9 \%$ and maximum of $499.8 \%$ (see Table 3.2). It was observed that 724 cases exist in which the RE of the proposed ratio estimator remained between $200.9 \%$ and $499.8 \%$.

Proposed Power Transformation Type Estimator

By following the repeated substitution method developed by Garcia and Cebrian (1996), consider a new power transformation ratio type estimator $\bar{y}_{\text {Power }}^{*}$ as:

$$
\bar{y}_{\text {Power }}^{*}=\bar{y}^{*}\left(\frac{\bar{x}_{1}^{*}}{\bar{x}_{2}^{*}}\right)^{\alpha}
$$

where $\alpha$ is a suitably chosen real constant. For example if $\alpha=0$ then the proposed power transformation ratio type estimator $\bar{y}_{\text {Power }}^{*}$ reduces to the $\mathrm{BBB}$ estimator $\bar{y}_{B B B}$. If $\alpha=1$ then the proposed power transformation ratio type estimator $\bar{y}_{\text {Power }}^{*}$ reduces to the ratio estimator $\bar{y}_{\text {Ratio }}^{*}$.

Note that the proposed transformation type estimator $\bar{y}_{\text {Power }}^{*}$ in terms of $\in, \delta$ and $\eta$ can be written as:

$$
\begin{aligned}
\bar{y}_{\text {Power }}^{*} & =\bar{Y}(1+\in)\left[\frac{\bar{X}(1+\delta)}{\bar{X}(1+\eta)}\right]^{\alpha} \\
& =\bar{Y}(1+\epsilon)\left[(1+\delta)(1+\eta)^{-1}\right]^{\alpha} \\
& =\bar{Y}(1+\epsilon)\left[(1+\delta)\left(1-\eta+\eta^{2}+\ldots\right)\right]^{\alpha} \\
& =\bar{Y}(1+\epsilon)\left[1+\delta-\eta+\delta^{2}-\delta \eta+\ldots\right]^{\alpha}
\end{aligned}
$$

$\left\{\right.$ step 4 assumes that $\left.\left|\delta-\eta+\delta^{2}-\delta \eta+\ldots\right|<1\right\}$ 


$$
\begin{aligned}
& =\bar{Y}(1+\in)\left[\begin{array}{l}
1+\alpha(\delta-\eta)+\alpha \delta^{2} \\
-\alpha \delta \eta+\ldots
\end{array}\right] \\
& =\bar{Y}\left[\begin{array}{l}
1+\epsilon+\alpha(\delta-\eta)+\alpha^{2} \delta^{2} \\
-\alpha \delta \eta+\alpha(\in \delta-\epsilon \eta)+\ldots
\end{array}\right] \\
& =\bar{Y}\left[\begin{array}{l}
1+\epsilon+\alpha(\delta-\eta)+\alpha^{2} \delta^{2} \\
-\alpha \delta \eta+\alpha \in \delta-\alpha \in \eta+\ldots
\end{array}\right]
\end{aligned}
$$

This leads to two additional theorems.

Theorem 4.1

The bias in the proposed power transformation ratio type estimator $\bar{y}_{\text {Power }}^{*}$ is given by:

$$
\begin{aligned}
& B\left(\bar{y}_{\text {Power }}^{*}\right)= \\
& \frac{\bar{Y}}{n}\left[\alpha^{2}\left\{C_{x_{1}}^{2}+\left(1+C_{x_{1}}^{2}\right) C_{p_{1}}^{2}\right\}\right. \\
& \quad-\alpha\left\{\frac{\rho_{X_{1} X_{2}} C_{x_{1}} C_{x_{2}}}{\left[p_{1}+\left(1-p_{1}\right) \theta_{1}\right]\left[p_{2}+\left(1-p_{2}\right) \theta_{2}\right]}\right. \\
& \quad-\frac{\rho_{Y X_{1}} C_{y} C_{x_{1}}}{[p+(1-p) \theta]\left[p_{1}+\left(1-p_{1}\right) \theta_{1}\right]} \\
&\left.+\frac{\rho_{Y X_{2}} C_{y} C_{x_{2}}}{\left.[p+(1-p) \theta]\left[p_{2}+\left(1-p_{2}\right) \theta_{2}\right]\right\}}\right]
\end{aligned}
$$

Theorem 4.1: Proof

Taking expected value on both sides of (4.2), and using

$$
B\left(\bar{y}_{\text {Power }}^{*}\right)=E\left(\bar{y}_{\text {Power }}^{*}\right)-\bar{Y}
$$

results in

$$
\begin{aligned}
B\left(\bar{y}_{\text {Power }}^{*}\right) & = \\
\frac{\bar{Y}}{n}\left[\alpha^{2}\left\{C_{x_{1}}^{2}+\left(1+C_{x_{1}}^{2}\right) C_{p_{1}}^{2}\right\}\right. & \\
& -\alpha\left\{\frac{\rho_{X_{1} X_{2}} C_{x_{1}} C_{x_{2}}}{\left[p_{1}+\left(1-p_{1}\right) \theta_{1}\right]\left[p_{2}+\left(1-p_{2}\right) \theta_{2}\right]}\right. \\
& -\frac{\rho_{Y X_{1}} C_{y} C_{x_{1}}}{[p+(1-p) \theta]\left[p_{1}+\left(1-p_{1}\right) \theta_{1}\right]} \\
& \left.\left.+\frac{\rho_{Y X_{2}} C_{y} C_{x_{2}}}{[p+(1-p) \theta]\left[p_{2}+\left(1-p_{2}\right) \theta_{2}\right]}\right\}\right]
\end{aligned}
$$

Theorem 4.2

The minimum mean squared error of the proposed power transformation ratio type estimator $\bar{y}_{\text {Power }}^{*}$ is given by formula (4.4) as shown below.

\section{Theorem 4.2: Proof}

By the definition of mean squared error,

$\operatorname{Min} \cdot \operatorname{MSE}\left(\bar{y}_{\text {Power }}^{*}\right)=$

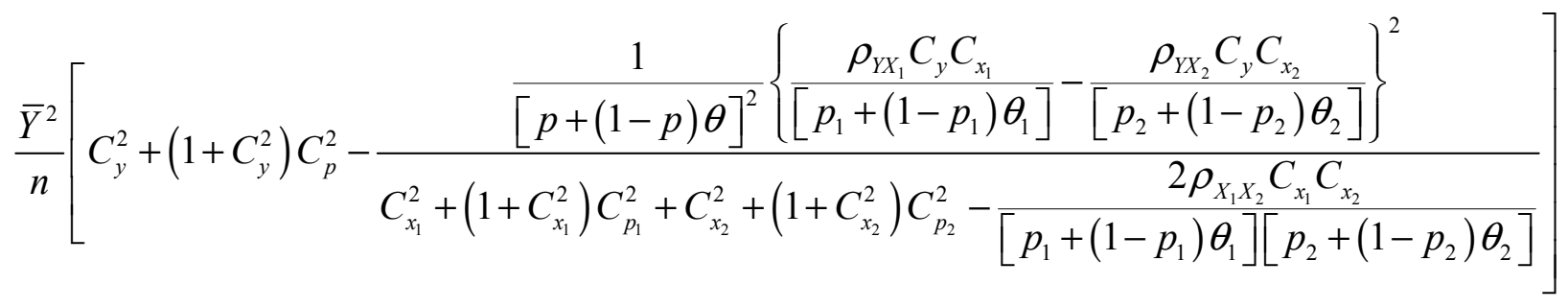




\section{ODUMADE \& SINGH}

$$
\begin{aligned}
& \operatorname{MSE}\left(\bar{y}_{\text {Power }}^{*}\right)=E\left[\bar{y}_{\text {Power }}^{*}-\bar{Y}\right]^{2} \\
& =\bar{Y}^{2} E[\epsilon+\alpha(\delta-\eta)]^{2} \\
& =\bar{Y}^{2} E\left[\begin{array}{l}
\epsilon^{2}+\alpha^{2}(\delta-\eta)^{2} \\
+2 \alpha \in(\delta-\eta)
\end{array}\right] \\
& =\bar{Y}^{2} E\left[\begin{array}{l}
\epsilon^{2}+\alpha^{2}\left(\delta^{2}+\eta^{2}-2 \delta \eta\right) \\
+2 \alpha(\in \delta-\epsilon \eta)
\end{array}\right] \\
& =\frac{\bar{Y}^{2}}{n}\left[C_{y}^{2}+\left(1+C_{y}^{2}\right) C_{p}^{2}\right. \\
& +\alpha^{2}\left\{\begin{array}{l}
C_{x_{1}}^{2}+\left(1+C_{x_{1}}^{2}\right) C_{p_{1}}^{2}+C_{x_{2}}^{2}+\left(1+C_{x_{2}}^{2}\right) C_{p_{2}}^{2} \\
-2 \frac{\rho_{X_{1} X_{2}} C_{x_{1}} C_{x_{2}}}{\left[p_{1}+\left(1-p_{1}\right) \theta_{1}\right]\left[p_{2}+\left(1-p_{2}\right) \theta_{2}\right]}
\end{array}\right\} \\
& +2 \alpha\left\{\begin{array}{c}
\frac{\rho_{Y X_{1}} C_{y} C_{x_{1}}}{[p+(1-p) \theta]\left[p_{1}+\left(1-p_{1}\right) \theta_{1}\right]} \\
-\frac{\rho_{Y X_{2}} C_{y} C_{x_{2}}}{[p+(1-p) \theta]\left[p_{2}+\left(1-p_{2}\right) \theta_{2}\right]}
\end{array}\right\}
\end{aligned}
$$

Differentiating (4.5) with respect to $\alpha$ and setting it equal to zero the optimum value of $\alpha$ as shown in Formula 4.6, results in the minimum MSE of $\bar{y}_{\text {Power }}^{*}$ as given by (4.4).

Based on these, it is clear that the proposed $\bar{y}_{\text {Power }}^{*}$ estimator remains more efficient than $\bar{y}_{B B B}$ for any choice of parameters in the proposed spinners or the design based parameters.

\section{Methodology}

Relative Efficiency of the Power Transformation Type Estimator with Respect to the BBB Model

In order to determine the magnitude of the proposed power transformation type estimator $\bar{y}_{\text {Power }}^{*}$ with respect to the $\mathrm{BBB}$ model the percent RE was computed as:

$$
\operatorname{RE}\left(\bar{y}_{\mathrm{BBB}}, \bar{y}_{\text {Power }}^{*}\right)=\frac{V\left(\bar{y}_{\mathrm{BBB}}\right)}{\operatorname{MSE}\left(\overline{\mathrm{y}}_{\text {Power }}^{*}\right)} \times 100 \%
$$

Again the RE of the power transformation estimator depends on parameters such as $P, P_{1}$, $P_{2}, C_{y}, C_{x_{1}}, C_{x_{2}}, C_{\gamma}, C_{\gamma_{1}}, C_{\gamma_{2}}, \theta, \theta_{1}$ and $\theta_{2}$; the percent RE is free from the sample size $n$ and main parameter of interest $\bar{Y}$. FORTRAN programs were developed in order to determine the values of the parameters $C_{y}$, $C_{x_{1}}, C_{x_{2}}, C_{\gamma}, C_{\gamma_{1}}, C_{\gamma_{2}}, \rho_{y x_{1}}, \rho_{y x_{2}}, \rho_{x_{1} x_{2}}$, $\theta, \theta_{1}$ and $\theta_{2}$ by holding $P, P_{1}$, and $P_{2}$ equal to 0.7 such that the percent RE remains higher than 200\% (see Table 4.1 in the Appendix for results).

The values of $C_{y}, C_{x_{1}}, C_{x_{2}}, C_{\gamma}$, $C_{\gamma_{1}}$, and $C_{\gamma_{2}}$ were changed between 0.1 and 0.5 with a step of 0.2 , and the values of $\theta, \theta_{1}$ and $\theta_{2}$ were changed between 0 and 1 with a step of 0.5 . It was observed that larger values of $\theta, \theta_{1}$ and $\theta_{2}$ may lead to slightly less efficient results, thus the choice of these values is critical when using the proposed power method in practice.

Table 4.2: Descriptive Statistics of the Percent Relative Efficiency

\begin{tabular}{|c|c|}
\hline Mean & 233.05 \\
\hline Standard Error & 7.33 \\
\hline Median & 215.47 \\
\hline Standard Deviation & 47.53 \\
\hline Sample Variance & 2258.76 \\
\hline Kurtosis & 4.65 \\
\hline Skewness & 2.34 \\
\hline Range & 178.60 \\
\hline Minimum & 200.61 \\
\hline Maximum & 379.21 \\
\hline Count & 42 \\
\hline
\end{tabular}

The values of $\rho_{y x_{2}}$ and $\rho_{x_{1} x_{2}}$ were changed between 0.1 and 0.9 with a step of 0.2 , and $\rho_{y x_{1}}$ 


$$
\begin{aligned}
& \alpha=\frac{-\frac{1}{[p+(1-p) \theta]}\left\{\frac{\rho_{Y X_{1}} C_{y} C_{x_{1}}}{\left[p_{1}+\left(1-p_{1}\right) \theta_{1}\right]}-\frac{\rho_{Y X_{2}} C_{y} C_{x_{2}}}{\left[p_{2}+\left(1-p_{2}\right) \theta_{2}\right]}\right\}}{C_{x_{1}}^{2}+\left(1+C_{x_{1}}^{2}\right) C_{p_{1}}^{2}+C_{x_{2}}^{2}+\left(1+C_{x_{2}}^{2}\right) C_{p_{2}}^{2}-\frac{C_{x_{1}} C_{x_{2}}}{\left[p_{1}+\left(1-p_{1}\right) \theta_{1}\right]\left[p_{2}+\left(1-p_{2}\right) \theta_{2}\right]}} \\
& \operatorname{MSE}\left(\bar{y}_{\text {Power }}^{*}\right)= \\
& \frac{\bar{Y}^{2}}{n}\left[C_{y}^{2}+\left(1+C_{y}^{2}\right) C_{p}^{2}-\frac{\frac{1}{[p+(1-p) \theta]^{2}}\left\{\frac{\rho_{Y X_{1}} C_{y} C_{x_{1}}}{\left[p_{1}+\left(1-p_{1}\right) \theta_{1}\right]}-\frac{\rho_{Y X_{2}} C_{y} C_{x_{2}}}{\left[p_{2}+\left(1-p_{2}\right) \theta_{2}\right]}\right\}^{2}}{C_{x_{1}}^{2}+\left(1+C_{x_{1}}^{2}\right) C_{p_{1}}^{2}+C_{x_{2}}^{2}+\left(1+C_{x_{2}}^{2}\right) C_{p_{2}}^{2}-\frac{C_{x_{1}} C_{x_{2}}}{\left[p_{1}+\left(1-p_{1}\right) \theta_{1}\right]\left[p_{2}+\left(1-p_{2}\right) \theta_{2}\right]}}\right]
\end{aligned}
$$

was changed between 0.1 to +0.9 with a step of 0.2 . The average percent relative efficiency was $233.5 \%$ with a standard deviation of 47.53 , a median of $215.47 \%$, minimum of $200.16 \%$ and maximum of $379.21 \%$ (see Table 4.2 ). It was observed that 42 cases exist where the RE of the proposed ratio estimator remained between $200.16 \%$ and $379.21 \%$. As shown in Table 4.1, the optimum values of $\alpha$ remained between 1.56 and +1.56 with a mean equal to zero, standard deviation of 0.93 and mode of 0.49 .

\section{Conclusion}

In this study new ratio and power transformation type estimators were proposed and compared to the recently described $\mathrm{BBB}$ randomized response model. It was observed that the overall magnitude of the relative efficiency of the ratio estimator - unlike the repeated substitution method due to Garcia and Cebrian (1996) - was better than that of the power transformation estimator in the case of scrambled responses.

\section{References}

Arnab, R. (1995). On admissibility and optimality of sampling strategies in randomized response surveys. Sankhy a , B57, 385-390.

Arnab, R. (1996). Randomized response trials: A unified approach for qualitative data. Communications in Statistics-Theory and Methods, 25(6), 1173-1183.

Bar-Lev, S. K., Bobovitch, E., \& Boukai, B. (2004). A note on randomized response models for quantitative data. Metrika, 255-260.

Bhargava, M. (1996). An investigation into the efficiencies of certain randomized response strategies. Unpublished Ph.D. thesis submitted to Punjab Agricultural University, Ludhiana, India.

Bhargava, M., \& Singh, R. (2001). Efficiency comparison of certain randomized response schemes with U-model. Journal of the Indian Society of Agricultural Statistics, 54(1), 19-28. 


\section{ODUMADE \& SINGH}

Bhargava, M., \& Singh, R. (2002). On the efficiency comparison of certain randomized response strategies. Metrika, 55(3), 191-197.

Eichhorn, B. H., \& Hayre, L. S. (1983). Scrambled randomized response methods for obtaining sensitive quantitative data. Journal of Statistical Planning and Inference, 7, 307-316.

Garcia, M. R., \& Cebrian, A. A. (1996). Repeated substitution method: The ratio estimator for the population variance. Metrika, 43, 101-105.

Gjestvang, C. R., \& Singh, S. (2006). A new randomized response model. Journal of the Royal Statistical Society, B68, 523-530.

Kim, J. M., \& Elam, M. E. (2005). A two-stage stratified Warner's randomized response model using optimal allocation. Metrika, 61, 1-7.

Kim, J.-M. and Warde, W. D. (2004). A stratified Warner's randomized response model. Journal of Statistical Planning and Inference, 120(1-2), 155-165.

Kim, J. M., \& Warde, W. D. (2005). A mixed randomized response model. Journal of Statistical Planning and Inference, 133, 211221.

Kuk, A. Y. C. (1990). Asking sensitive questions indirectly. Biometrika, 77, 436-438.

Lanke, J. (1975). On the choice of the unrelated question in Simons version of randomized response. Journal of the American Statistical Association, 70, 80-83.

Lanke, J. (1976). On the degree of protection in randomized interviews. International Statistical Review, 44, 197-203.

Leysieffer, F. W., \& Warner, S. L. (1976). Respondent jeopardy and optimal designs in randomized response models. Journal of the American Statistical Association, 71, 649656.
Mangat, N. S. (1994). An improved randomized response strategy. Journal of the Royal Statistical Society, B56, 93-95.

Mangat, N. S., \& Singh, R. (1990). An alternative randomized response procedure. Biometrika, 77(2), 439-442.

Moors, J. J. A. (1997). A critical evaluation of Mangat's two-step procedure in randomized response. Discussion paper at Center for Economic Research, Tilburg University, The Netherlands.

Nayak, T. K. (1994). On randomized response surveys for estimating a proportion. Communications in Statistics-Theory and Methods, 23(1), 3303-3321.

Singh, H. P., \& Mathur, N. (2005). Estimation of population mean when coefficient of variation is known using scrambled response technique. Journal of Statistical Planning and Inference, 131, 135-144.

Singh, S. (2003). Advanced Sampling Theory with Applications: How Michael "Selected" Amy. The Netherlands: Kluwer Academic Publishers.

Tracy, D. S., \& Mangat, N. S. (1996). Some developments in randomized response sampling during the last decade: A follow up of review by Chaudhuri and Mukerjee. Journal of Applied Statistical Science, 4(2/3), 147-158.

Tripathi, T. P., \& Chaubey, Y. P. (1992). Improved estimation of a finite population mean based on paired observations. Communications in Statistics-Theory and Methods, 21, 3327-3333.

Warner, S. L. (1965). Randomized response: A survey technique for eliminating evasive answer bias. Journal of the American Statistical Association, 60, 63-69. 


\section{Appendix}

Table 3.1: Relative efficiency of the proposed ratio estimator with respect to the BBB model for different choice of parameters with $P=P_{1}=P_{2}=0.7$

\begin{tabular}{|c|c|c|c|c|c|c|c|c|c|c|c|c|}
\hline$C_{\gamma}$ & $C_{\gamma_{1}}$ & $C_{\gamma_{2}}$ & $C_{y}$ & $C x_{1}$ & $C x_{2}$ & $\rho_{y x_{1}}$ & $\rho_{y x_{2}}$ & $\rho_{x_{1} x_{2}}$ & $\theta$ & $\theta_{1}$ & $\theta_{2}$ & $\mathrm{RE}$ \\
\hline 0.1 & 0.1 & 0.1 & 0.5 & 0.5 & 0.3 & -0.9 & 0.7 & 0.7 & 0.0 & 0.0 & 0.0 & 222.54 \\
\hline 0.1 & 0.1 & 0.1 & 0.5 & 0.5 & 0.5 & -0.9 & 0.5 & 0.7 & 0.0 & 0.0 & 0.0 & 366.67 \\
\hline 0.1 & 0.1 & 0.1 & 0.5 & 0.5 & 0.5 & -0.9 & 0.7 & 0.5 & 0.0 & 0.0 & 0.0 & 366.67 \\
\hline 0.1 & 0.1 & 0.1 & 0.5 & 0.5 & 0.5 & -0.7 & 0.7 & 0.7 & 0.0 & 0.0 & 0.0 & 366.67 \\
\hline 0.3 & 0.3 & 0.3 & 0.5 & 0.5 & 0.3 & -0.9 & 0.7 & 0.7 & 0.0 & 0.0 & 0.0 & 222.54 \\
\hline 0.3 & 0.3 & 0.3 & 0.5 & 0.5 & 0.5 & -0.9 & 0.5 & 0.7 & 0.0 & 0.0 & 0.0 & 366.67 \\
\hline 0.3 & 0.3 & 0.3 & 0.5 & 0.5 & 0.5 & -0.9 & 0.7 & 0.5 & 0.0 & 0.0 & 0.0 & 366.67 \\
\hline 0.3 & 0.3 & 0.3 & 0.5 & 0.5 & 0.5 & -0.7 & 0.7 & 0.7 & 0.0 & 0.0 & 0.0 & 366.67 \\
\hline 0.5 & 0.5 & 0.5 & 0.5 & 0.5 & 0.3 & -0.9 & 0.7 & 0.7 & 0.0 & 0.0 & 0.0 & 222.54 \\
\hline 0.5 & 0.5 & 0.5 & 0.5 & 0.5 & 0.5 & -0.9 & 0.5 & 0.7 & 0.0 & 0.0 & 0.0 & 366.67 \\
\hline 0.5 & 0.5 & 0.5 & 0.5 & 0.5 & 0.5 & -0.9 & 0.7 & 0.5 & 0.0 & 0.0 & 0.0 & 366.67 \\
\hline 0.5 & 0.5 & 0.5 & 0.5 & 0.5 & 0.5 & -0.7 & 0.7 & 0.7 & 0.0 & 0.0 & 0.0 & 366.67 \\
\hline 0.1 & 0.1 & 0.1 & 0.3 & 0.3 & 0.1 & -0.9 & 0.7 & 0.7 & 0.5 & 0.5 & 0.5 & 201.28 \\
\hline 0.1 & 0.1 & 0.1 & 0.3 & 0.3 & 0.3 & -0.9 & 0.3 & 0.7 & 0.5 & 0.5 & 0.5 & 452.41 \\
\hline 0.1 & 0.1 & 0.1 & 0.3 & 0.3 & 0.3 & -0.9 & 0.5 & 0.5 & 0.5 & 0.5 & 0.5 & 452.41 \\
\hline 0.1 & 0.1 & 0.1 & 0.3 & 0.3 & 0.3 & -0.9 & 0.7 & 0.3 & 0.5 & 0.5 & 0.5 & 452.41 \\
\hline 0.1 & 0.1 & 0.1 & 0.3 & 0.3 & 0.3 & -0.7 & 0.5 & 0.7 & 0.5 & 0.5 & 0.5 & 452.41 \\
\hline 0.1 & 0.1 & 0.1 & 0.3 & 0.3 & 0.3 & -0.7 & 0.7 & 0.5 & 0.5 & 0.5 & 0.5 & 452.41 \\
\hline 0.1 & 0.1 & 0.1 & 0.3 & 0.3 & 0.3 & -0.5 & 0.7 & 0.7 & 0.5 & 0.5 & 0.5 & 452.41 \\
\hline 0.1 & 0.1 & 0.1 & 0.3 & 0.3 & 0.5 & -0.9 & 0.3 & 0.7 & 0.5 & 0.5 & 0.5 & 392.90 \\
\hline 0.1 & 0.1 & 0.1 & 0.3 & 0.3 & 0.5 & -0.9 & 0.5 & 0.5 & 0.5 & 0.5 & 0.5 & 392.90 \\
\hline 0.1 & 0.1 & 0.1 & 0.3 & 0.3 & 0.5 & -0.9 & 0.7 & 0.3 & 0.5 & 0.5 & 0.5 & 392.90 \\
\hline 0.1 & 0.1 & 0.1 & 0.3 & 0.3 & 0.5 & -0.5 & 0.5 & 0.7 & 0.5 & 0.5 & 0.5 & 284.07 \\
\hline 0.1 & 0.1 & 0.1 & 0.3 & 0.3 & 0.5 & -0.5 & 0.7 & 0.5 & 0.5 & 0.5 & 0.5 & 284.07 \\
\hline 0.1 & 0.1 & 0.1 & 0.3 & 0.3 & 0.5 & -0.1 & 0.7 & 0.7 & 0.5 & 0.5 & 0.5 & 222.45 \\
\hline 0.1 & 0.1 & 0.1 & 0.3 & 0.5 & 0.1 & -0.9 & 0.5 & 0.7 & 0.5 & 0.5 & 0.5 & 200.89 \\
\hline 0.1 & 0.1 & 0.1 & 0.3 & 0.5 & 0.1 & -0.9 & 0.7 & 0.7 & 0.5 & 0.5 & 0.5 & 249.82 \\
\hline 0.1 & 0.1 & 0.1 & 0.3 & 0.5 & 0.3 & -0.9 & 0.1 & 0.5 & 0.5 & 0.5 & 0.5 & 222.45 \\
\hline 0.1 & 0.1 & 0.1 & 0.3 & 0.5 & 0.3 & -0.9 & 0.5 & 0.3 & 0.5 & 0.5 & 0.5 & 284.07 \\
\hline 0.1 & 0.1 & 0.1 & 0.3 & 0.5 & 0.3 & -0.7 & 0.1 & 0.7 & 0.5 & 0.5 & 0.5 & 222.45 \\
\hline 0.1 & 0.1 & 0.1 & 0.3 & 0.5 & 0.3 & -0.7 & 0.5 & 0.5 & 0.5 & 0.5 & 0.5 & 284.07 \\
\hline 0.1 & 0.1 & 0.1 & 0.3 & 0.5 & 0.3 & -0.5 & 0.5 & 0.7 & 0.5 & 0.5 & 0.5 & 284.07 \\
\hline 0.1 & 0.1 & 0.1 & 0.3 & 0.5 & 0.5 & -0.9 & 0.5 & 0.3 & 0.5 & 0.5 & 0.5 & 259.40 \\
\hline 0.1 & 0.1 & 0.1 & 0.3 & 0.5 & 0.5 & -0.7 & 0.1 & 0.7 & 0.5 & 0.5 & 0.5 & 448.46 \\
\hline 0.1 & 0.1 & 0.1 & 0.3 & 0.5 & 0.5 & -0.7 & 0.7 & 0.3 & 0.5 & 0.5 & 0.5 & 259.40 \\
\hline 0.1 & 0.1 & 0.1 & 0.3 & 0.5 & 0.5 & -0.5 & 0.3 & 0.7 & 0.5 & 0.5 & 0.5 & 448.46 \\
\hline 0.1 & 0.1 & 0.1 & 0.3 & 0.5 & 0.5 & -0.3 & 0.5 & 0.7 & 0.5 & 0.5 & 0.5 & 448.46 \\
\hline 0.1 & 0.1 & 0.1 & 0.3 & 0.5 & 0.5 & -0.1 & 0.7 & 0.7 & 0.5 & 0.5 & 0.5 & 448.46 \\
\hline 0.1 & 0.1 & 0.1 & 0.5 & 0.1 & 0.3 & -0.9 & 0.7 & 0.3 & 0.5 & 0.5 & 0.5 & 218.24 \\
\hline 0.1 & 0.1 & 0.1 & 0.5 & 0.1 & 0.3 & -0.9 & 0.7 & 0.5 & 0.5 & 0.5 & 0.5 & 244.11 \\
\hline 0.1 & 0.1 & 0.1 & 0.5 & 0.1 & 0.3 & -0.9 & 0.7 & 0.7 & 0.5 & 0.5 & 0.5 & 276.92 \\
\hline
\end{tabular}


ODUMADE \& SINGH

Appendix

Table 3.1 (continued): Relative efficiency of the proposed ratio estimator with respect to the BBB model for different choice of parameters with $P=P_{1}=P_{2}=0.7$

\begin{tabular}{|c|c|c|c|c|c|c|c|c|c|c|c|c|}
\hline$C_{\gamma}$ & $C_{\gamma_{1}}$ & $C_{\gamma_{2}}$ & $C_{y}$ & $C x_{1}$ & $C x_{2}$ & $\rho_{y x_{1}}$ & $\rho_{y x_{2}}$ & $\rho_{x_{1} x_{2}}$ & $\theta$ & $\theta_{1}$ & $\theta_{2}$ & $\mathrm{RE}$ \\
\hline 0.1 & 0.1 & 0.1 & 0.5 & 0.1 & 0.3 & -0.7 & 0.7 & 0.5 & 0.5 & 0.5 & 0.5 & 203.84 \\
\hline 0.1 & 0.1 & 0.1 & 0.5 & 0.1 & 0.3 & -0.7 & 0.7 & 0.7 & 0.5 & 0.5 & 0.5 & 226.23 \\
\hline 0.1 & 0.1 & 0.1 & 0.5 & 0.1 & 0.5 & -0.9 & 0.7 & 0.1 & 0.5 & 0.5 & 0.5 & 234.56 \\
\hline 0.1 & 0.1 & 0.1 & 0.5 & 0.1 & 0.5 & -0.9 & 0.7 & 0.3 & 0.5 & 0.5 & 0.5 & 289.51 \\
\hline 0.1 & 0.1 & 0.1 & 0.5 & 0.1 & 0.5 & -0.9 & 0.7 & 0.5 & 0.5 & 0.5 & 0.5 & 378.06 \\
\hline 0.1 & 0.1 & 0.1 & 0.5 & 0.1 & 0.5 & -0.7 & 0.7 & 0.3 & 0.5 & 0.5 & 0.5 & 234.56 \\
\hline 0.1 & 0.1 & 0.1 & 0.5 & 0.1 & 0.5 & -0.7 & 0.7 & 0.5 & 0.5 & 0.5 & 0.5 & 289.51 \\
\hline 0.1 & 0.1 & 0.1 & 0.5 & 0.1 & 0.5 & -0.7 & 0.7 & 0.7 & 0.5 & 0.5 & 0.5 & 378.06 \\
\hline 0.1 & 0.1 & 0.1 & 0.5 & 0.1 & 0.5 & -0.5 & 0.7 & 0.5 & 0.5 & 0.5 & 0.5 & 234.56 \\
\hline 0.1 & 0.1 & 0.1 & 0.5 & 0.1 & 0.5 & -0.5 & 0.7 & 0.7 & 0.5 & 0.5 & 0.5 & 289.51 \\
\hline 0.1 & 0.1 & 0.1 & 0.5 & 0.1 & 0.5 & -0.3 & 0.7 & 0.7 & 0.5 & 0.5 & 0.5 & 234.56 \\
\hline 0.1 & 0.1 & 0.1 & 0.5 & 0.3 & 0.1 & -0.9 & 0.1 & 0.5 & 0.5 & 0.5 & 0.5 & 203.84 \\
\hline 0.1 & 0.1 & 0.1 & 0.5 & 0.3 & 0.1 & -0.9 & 0.1 & 0.7 & 0.5 & 0.5 & 0.5 & 226.23 \\
\hline 0.1 & 0.1 & 0.1 & 0.5 & 0.3 & 0.1 & -0.9 & 0.3 & 0.3 & 0.5 & 0.5 & 0.5 & 218.24 \\
\hline 0.1 & 0.1 & 0.1 & 0.5 & 0.3 & 0.1 & -0.9 & 0.3 & 0.5 & 0.5 & 0.5 & 0.5 & 244.11 \\
\hline 0.1 & 0.1 & 0.1 & 0.5 & 0.3 & 0.1 & -0.9 & 0.3 & 0.7 & 0.5 & 0.5 & 0.5 & 276.92 \\
\hline 0.1 & 0.1 & 0.1 & 0.5 & 0.3 & 0.1 & -0.9 & 0.5 & 0.1 & 0.5 & 0.5 & 0.5 & 234.83 \\
\hline 0.1 & 0.1 & 0.1 & 0.5 & 0.3 & 0.1 & -0.9 & 0.5 & 0.3 & 0.5 & 0.5 & 0.5 & 265.04 \\
\hline 0.1 & 0.1 & 0.1 & 0.5 & 0.3 & 0.1 & -0.9 & 0.5 & 0.5 & 0.5 & 0.5 & 0.5 & 304.18 \\
\hline 0.1 & 0.1 & 0.1 & 0.5 & 0.3 & 0.1 & -0.9 & 0.5 & 0.7 & 0.5 & 0.5 & 0.5 & 356.89 \\
\hline 0.1 & 0.1 & 0.1 & 0.5 & 0.3 & 0.1 & -0.9 & 0.7 & 0.1 & 0.5 & 0.5 & 0.5 & 289.91 \\
\hline 0.1 & 0.1 & 0.1 & 0.5 & 0.3 & 0.1 & -0.9 & 0.7 & 0.3 & 0.5 & 0.5 & 0.5 & 337.40 \\
\hline 0.1 & 0.1 & 0.1 & 0.5 & 0.3 & 0.1 & -0.9 & 0.7 & 0.5 & 0.5 & 0.5 & 0.5 & 403.49 \\
\hline 0.1 & 0.1 & 0.1 & 0.5 & 0.3 & 0.1 & -0.7 & 0.7 & 0.5 & 0.5 & 0.5 & 0.5 & 203.84 \\
\hline 0.1 & 0.1 & 0.1 & 0.5 & 0.3 & 0.1 & -0.7 & 0.7 & 0.7 & 0.5 & 0.5 & 0.5 & 226.23 \\
\hline 0.1 & 0.1 & 0.1 & 0.5 & 0.3 & 0.3 & -0.9 & 0.1 & 0.5 & 0.5 & 0.5 & 0.5 & 239.24 \\
\hline 0.1 & 0.1 & 0.1 & 0.5 & 0.3 & 0.3 & -0.9 & 0.1 & 0.7 & 0.5 & 0.5 & 0.5 & 367.17 \\
\hline 0.1 & 0.1 & 0.1 & 0.5 & 0.3 & 0.3 & -0.9 & 0.3 & 0.1 & 0.5 & 0.5 & 0.5 & 214.34 \\
\hline 0.1 & 0.1 & 0.1 & 0.5 & 0.3 & 0.3 & -0.9 & 0.3 & 0.3 & 0.5 & 0.5 & 0.5 & 311.62 \\
\hline 0.1 & 0.1 & 0.1 & 0.5 & 0.3 & 0.3 & -0.9 & 0.5 & 0.1 & 0.5 & 0.5 & 0.5 & 446.81 \\
\hline 0.1 & 0.1 & 0.1 & 0.5 & 0.3 & 0.3 & -0.7 & 0.3 & 0.5 & 0.5 & 0.5 & 0.5 & 239.24 \\
\hline 0.1 & 0.1 & 0.1 & 0.5 & 0.3 & 0.3 & -0.7 & 0.3 & 0.7 & 0.5 & 0.5 & 0.5 & 367.17 \\
\hline 0.1 & 0.1 & 0.1 & 0.5 & 0.3 & 0.3 & -0.7 & 0.5 & 0.1 & 0.5 & 0.5 & 0.5 & 214.34 \\
\hline 0.1 & 0.1 & 0.1 & 0.5 & 0.3 & 0.3 & -0.7 & 0.5 & 0.3 & 0.5 & 0.5 & 0.5 & 311.62 \\
\hline 0.1 & 0.1 & 0.1 & 0.5 & 0.3 & 0.3 & -0.7 & 0.7 & 0.1 & 0.5 & 0.5 & 0.5 & 446.81 \\
\hline 0.1 & 0.1 & 0.1 & 0.5 & 0.3 & 0.3 & -0.5 & 0.5 & 0.5 & 0.5 & 0.5 & 0.5 & 239.24 \\
\hline 0.1 & 0.1 & 0.1 & 0.5 & 0.3 & 0.3 & -0.5 & 0.5 & 0.7 & 0.5 & 0.5 & 0.5 & 367.17 \\
\hline 0.1 & 0.1 & 0.1 & 0.5 & 0.3 & 0.3 & -0.5 & 0.7 & 0.1 & 0.5 & 0.5 & 0.5 & 214.34 \\
\hline 0.1 & 0.1 & 0.1 & 0.5 & 0.3 & 0.3 & -0.5 & 0.7 & 0.3 & 0.5 & 0.5 & 0.5 & 311.62 \\
\hline 0.1 & 0.1 & 0.1 & 0.5 & 0.3 & 0.3 & -0.3 & 0.7 & 0.5 & 0.5 & 0.5 & 0.5 & 239.24 \\
\hline 0.1 & 0.1 & 0.1 & 0.5 & 0.3 & 0.3 & -0.3 & 0.7 & 0.7 & 0.5 & 0.5 & 0.5 & 367.17 \\
\hline
\end{tabular}


IMPROVING THE BAR-LEV, BOBOVITCH \& BOUKAI RANDOM RESPONSE MODELS

\section{Appendix}

Table 3.1 (continued): Relative efficiency of the proposed ratio estimator with respect to the BBB model for different choice of parameters with $P=P_{1}=P_{2}=0.7$

\begin{tabular}{|c|c|c|c|c|c|c|c|c|c|c|c|c|}
\hline$C_{\gamma}$ & $C_{\gamma_{1}}$ & $C_{\gamma_{2}}$ & $C_{y}$ & $C x_{1}$ & $C x_{2}$ & $\rho_{y x_{1}}$ & $\rho_{y x_{2}}$ & $\rho_{x_{1} x_{2}}$ & $\theta$ & $\theta_{1}$ & $\theta_{2}$ & $\mathrm{RE}$ \\
\hline 0.1 & 0.1 & 0.1 & 0.5 & 0.3 & 0.5 & -0.9 & 0.1 & 0.7 & 0.5 & 0.5 & 0.5 & 282.69 \\
\hline 0.1 & 0.1 & 0.1 & 0.5 & 0.3 & 0.5 & -0.9 & 0.3 & 0.3 & 0.5 & 0.5 & 0.5 & 230.06 \\
\hline 0.1 & 0.1 & 0.1 & 0.5 & 0.3 & 0.5 & -0.9 & 0.5 & 0.1 & 0.5 & 0.5 & 0.5 & 366.52 \\
\hline 0.1 & 0.1 & 0.1 & 0.5 & 0.3 & 0.5 & -0.7 & 0.3 & 0.5 & 0.5 & 0.5 & 0.5 & 230.06 \\
\hline 0.1 & 0.1 & 0.1 & 0.5 & 0.3 & 0.5 & -0.7 & 0.5 & 0.3 & 0.5 & 0.5 & 0.5 & 366.52 \\
\hline 0.1 & 0.1 & 0.1 & 0.5 & 0.3 & 0.5 & -0.5 & 0.3 & 0.7 & 0.5 & 0.5 & 0.5 & 230.06 \\
\hline 0.1 & 0.1 & 0.1 & 0.5 & 0.3 & 0.5 & -0.5 & 0.5 & 0.5 & 0.5 & 0.5 & 0.5 & 366.52 \\
\hline 0.1 & 0.1 & 0.1 & 0.5 & 0.3 & 0.5 & -0.5 & 0.7 & 0.1 & 0.5 & 0.5 & 0.5 & 282.69 \\
\hline 0.1 & 0.1 & 0.1 & 0.5 & 0.3 & 0.5 & -0.3 & 0.5 & 0.7 & 0.5 & 0.5 & 0.5 & 366.52 \\
\hline 0.1 & 0.1 & 0.1 & 0.5 & 0.3 & 0.5 & -0.3 & 0.7 & 0.3 & 0.5 & 0.5 & 0.5 & 282.69 \\
\hline 0.1 & 0.1 & 0.1 & 0.5 & 0.3 & 0.5 & -0.1 & 0.7 & 0.5 & 0.5 & 0.5 & 0.5 & 282.69 \\
\hline 0.1 & 0.1 & 0.1 & 0.5 & 0.3 & 0.5 & 0.1 & 0.7 & 0.7 & 0.5 & 0.5 & 0.5 & 282.69 \\
\hline 0.1 & 0.1 & 0.1 & 0.5 & 0.5 & 0.1 & -0.9 & 0.1 & 0.1 & 0.5 & 0.5 & 0.5 & 289.51 \\
\hline 0.1 & 0.1 & 0.1 & 0.5 & 0.5 & 0.1 & -0.9 & 0.1 & 0.3 & 0.5 & 0.5 & 0.5 & 378.06 \\
\hline 0.1 & 0.1 & 0.1 & 0.5 & 0.5 & 0.1 & -0.9 & 0.3 & 0.1 & 0.5 & 0.5 & 0.5 & 378.06 \\
\hline 0.1 & 0.1 & 0.1 & 0.5 & 0.5 & 0.1 & -0.7 & 0.3 & 0.7 & 0.5 & 0.5 & 0.5 & 234.56 \\
\hline 0.1 & 0.1 & 0.1 & 0.5 & 0.5 & 0.1 & -0.7 & 0.5 & 0.5 & 0.5 & 0.5 & 0.5 & 234.56 \\
\hline 0.1 & 0.1 & 0.1 & 0.5 & 0.5 & 0.1 & -0.7 & 0.5 & 0.7 & 0.5 & 0.5 & 0.5 & 289.51 \\
\hline 0.1 & 0.1 & 0.1 & 0.5 & 0.5 & 0.1 & -0.7 & 0.7 & 0.3 & 0.5 & 0.5 & 0.5 & 234.56 \\
\hline 0.1 & 0.1 & 0.1 & 0.5 & 0.5 & 0.1 & -0.7 & 0.7 & 0.5 & 0.5 & 0.5 & 0.5 & 289.51 \\
\hline 0.1 & 0.1 & 0.1 & 0.5 & 0.5 & 0.1 & -0.7 & 0.7 & 0.7 & 0.5 & 0.5 & 0.5 & 378.06 \\
\hline 0.1 & 0.1 & 0.1 & 0.5 & 0.5 & 0.3 & -0.9 & 0.1 & 0.1 & 0.5 & 0.5 & 0.5 & 230.06 \\
\hline 0.1 & 0.1 & 0.1 & 0.5 & 0.5 & 0.3 & -0.7 & 0.1 & 0.5 & 0.5 & 0.5 & 0.5 & 282.69 \\
\hline 0.1 & 0.1 & 0.1 & 0.5 & 0.5 & 0.3 & -0.7 & 0.3 & 0.3 & 0.5 & 0.5 & 0.5 & 282.69 \\
\hline 0.1 & 0.1 & 0.1 & 0.5 & 0.5 & 0.3 & -0.7 & 0.5 & 0.1 & 0.5 & 0.5 & 0.5 & 282.69 \\
\hline 0.1 & 0.1 & 0.1 & 0.5 & 0.5 & 0.3 & -0.5 & 0.3 & 0.7 & 0.5 & 0.5 & 0.5 & 366.52 \\
\hline 0.1 & 0.1 & 0.1 & 0.5 & 0.5 & 0.3 & -0.5 & 0.5 & 0.5 & 0.5 & 0.5 & 0.5 & 366.52 \\
\hline 0.1 & 0.1 & 0.1 & 0.5 & 0.5 & 0.3 & -0.5 & 0.7 & 0.3 & 0.5 & 0.5 & 0.5 & 366.52 \\
\hline 0.1 & 0.1 & 0.1 & 0.5 & 0.5 & 0.3 & -0.3 & 0.5 & 0.7 & 0.5 & 0.5 & 0.5 & 230.06 \\
\hline 0.1 & 0.1 & 0.1 & 0.5 & 0.5 & 0.3 & -0.3 & 0.7 & 0.5 & 0.5 & 0.5 & 0.5 & 230.06 \\
\hline 0.1 & 0.1 & 0.1 & 0.5 & 0.5 & 0.5 & -0.9 & 0.1 & 0.3 & 0.5 & 0.5 & 0.5 & 269.97 \\
\hline 0.1 & 0.1 & 0.1 & 0.5 & 0.5 & 0.5 & -0.9 & 0.3 & 0.1 & 0.5 & 0.5 & 0.5 & 269.97 \\
\hline 0.1 & 0.1 & 0.1 & 0.5 & 0.5 & 0.5 & -0.7 & 0.1 & 0.5 & 0.5 & 0.5 & 0.5 & 269.97 \\
\hline 0.1 & 0.1 & 0.1 & 0.5 & 0.5 & 0.5 & -0.7 & 0.3 & 0.3 & 0.5 & 0.5 & 0.5 & 269.97 \\
\hline 0.1 & 0.1 & 0.1 & 0.5 & 0.5 & 0.5 & -0.7 & 0.5 & 0.1 & 0.5 & 0.5 & 0.5 & 269.97 \\
\hline 0.1 & 0.1 & 0.1 & 0.5 & 0.5 & 0.5 & -0.5 & 0.1 & 0.7 & 0.5 & 0.5 & 0.5 & 269.97 \\
\hline 0.1 & 0.1 & 0.1 & 0.5 & 0.5 & 0.5 & -0.5 & 0.3 & 0.5 & 0.5 & 0.5 & 0.5 & 269.97 \\
\hline 0.1 & 0.1 & 0.1 & 0.5 & 0.5 & 0.5 & -0.5 & 0.5 & 0.3 & 0.5 & 0.5 & 0.5 & 269.97 \\
\hline 0.1 & 0.1 & 0.1 & 0.5 & 0.5 & 0.5 & -0.5 & 0.7 & 0.1 & 0.5 & 0.5 & 0.5 & 269.97 \\
\hline 0.1 & 0.1 & 0.1 & 0.5 & 0.5 & 0.5 & -0.3 & 0.3 & 0.7 & 0.5 & 0.5 & 0.5 & 269.97 \\
\hline 0.1 & 0.1 & 0.1 & 0.5 & 0.5 & 0.5 & -0.3 & 0.5 & 0.5 & 0.5 & 0.5 & 0.5 & 269.97 \\
\hline
\end{tabular}




\section{ODUMADE \& SINGH}

Appendix

Table 3.1 (continued): Relative efficiency of the proposed ratio estimator with respect to the BBB model for different choice of parameters with $P=P_{1}=P_{2}=0.7$

\begin{tabular}{|c|c|c|c|c|c|c|c|c|c|c|c|c|}
\hline$C_{\gamma}$ & $C_{\gamma_{1}}$ & $C_{\gamma_{2}}$ & $C_{y}$ & $C x_{1}$ & $C x_{2}$ & $\rho_{y x_{1}}$ & $\rho_{y x_{2}}$ & $\rho_{x_{1} x_{2}}$ & $\theta$ & $\theta_{1}$ & $\theta_{2}$ & $\mathrm{RE}$ \\
\hline 0.1 & 0.1 & 0.1 & 0.5 & 0.5 & 0.5 & -0.3 & 0.7 & 0.3 & 0.5 & 0.5 & 0.5 & 269.97 \\
\hline 0.1 & 0.1 & 0.1 & 0.5 & 0.5 & 0.5 & -0.1 & 0.5 & 0.7 & 0.5 & 0.5 & 0.5 & 269.97 \\
\hline 0.1 & 0.1 & 0.1 & 0.5 & 0.5 & 0.5 & -0.1 & 0.7 & 0.5 & 0.5 & 0.5 & 0.5 & 269.97 \\
\hline 0.1 & 0.1 & 0.1 & 0.5 & 0.5 & 0.5 & 0.1 & 0.7 & 0.7 & 0.5 & 0.5 & 0.5 & 269.97 \\
\hline 0.3 & 0.3 & 0.3 & 0.3 & 0.3 & 0.3 & -0.9 & 0.3 & 0.7 & 0.5 & 0.5 & 0.5 & 276.81 \\
\hline 0.3 & 0.3 & 0.3 & 0.3 & 0.3 & 0.3 & -0.9 & 0.5 & 0.5 & 0.5 & 0.5 & 0.5 & 276.81 \\
\hline 0.3 & 0.3 & 0.3 & 0.3 & 0.3 & 0.3 & -0.9 & 0.7 & 0.3 & 0.5 & 0.5 & 0.5 & 276.81 \\
\hline 0.3 & 0.3 & 0.3 & 0.3 & 0.3 & 0.3 & -0.7 & 0.5 & 0.7 & 0.5 & 0.5 & 0.5 & 276.81 \\
\hline 0.3 & 0.3 & 0.3 & 0.3 & 0.3 & 0.3 & -0.7 & 0.7 & 0.5 & 0.5 & 0.5 & 0.5 & 276.81 \\
\hline 0.3 & 0.3 & 0.3 & 0.3 & 0.3 & 0.3 & -0.5 & 0.7 & 0.7 & 0.5 & 0.5 & 0.5 & 276.81 \\
\hline 0.3 & 0.3 & 0.3 & 0.3 & 0.3 & 0.5 & -0.9 & 0.3 & 0.7 & 0.5 & 0.5 & 0.5 & 249.71 \\
\hline 0.3 & 0.3 & 0.3 & 0.3 & 0.3 & 0.5 & -0.9 & 0.5 & 0.5 & 0.5 & 0.5 & 0.5 & 249.71 \\
\hline 0.3 & 0.3 & 0.3 & 0.3 & 0.3 & 0.5 & -0.9 & 0.7 & 0.3 & 0.5 & 0.5 & 0.5 & 249.71 \\
\hline 0.3 & 0.3 & 0.3 & 0.3 & 0.3 & 0.5 & -0.7 & 0.5 & 0.7 & 0.5 & 0.5 & 0.5 & 464.50 \\
\hline 0.3 & 0.3 & 0.3 & 0.3 & 0.3 & 0.5 & -0.7 & 0.7 & 0.5 & 0.5 & 0.5 & 0.5 & 464.50 \\
\hline 0.3 & 0.3 & 0.3 & 0.3 & 0.3 & 0.5 & -0.5 & 0.5 & 0.7 & 0.5 & 0.5 & 0.5 & 202.82 \\
\hline 0.3 & 0.3 & 0.3 & 0.3 & 0.3 & 0.5 & -0.5 & 0.7 & 0.5 & 0.5 & 0.5 & 0.5 & 202.82 \\
\hline 0.3 & 0.3 & 0.3 & 0.3 & 0.3 & 0.5 & -0.3 & 0.7 & 0.7 & 0.5 & 0.5 & 0.5 & 324.81 \\
\hline 0.3 & 0.3 & 0.3 & 0.3 & 0.5 & 0.3 & -0.9 & 0.3 & 0.5 & 0.5 & 0.5 & 0.5 & 324.81 \\
\hline 0.3 & 0.3 & 0.3 & 0.3 & 0.5 & 0.3 & -0.9 & 0.5 & 0.3 & 0.5 & 0.5 & 0.5 & 202.82 \\
\hline 0.3 & 0.3 & 0.3 & 0.3 & 0.5 & 0.3 & -0.9 & 0.7 & 0.3 & 0.5 & 0.5 & 0.5 & 464.50 \\
\hline 0.3 & 0.3 & 0.3 & 0.3 & 0.5 & 0.3 & -0.7 & 0.3 & 0.7 & 0.5 & 0.5 & 0.5 & 324.81 \\
\hline 0.3 & 0.3 & 0.3 & 0.3 & 0.5 & 0.3 & -0.7 & 0.5 & 0.5 & 0.5 & 0.5 & 0.5 & 202.82 \\
\hline 0.3 & 0.3 & 0.3 & 0.3 & 0.5 & 0.3 & -0.7 & 0.7 & 0.5 & 0.5 & 0.5 & 0.5 & 464.50 \\
\hline 0.3 & 0.3 & 0.3 & 0.3 & 0.5 & 0.3 & -0.5 & 0.5 & 0.7 & 0.5 & 0.5 & 0.5 & 202.82 \\
\hline 0.3 & 0.3 & 0.3 & 0.3 & 0.5 & 0.3 & -0.5 & 0.7 & 0.7 & 0.5 & 0.5 & 0.5 & 464.50 \\
\hline 0.3 & 0.3 & 0.3 & 0.3 & 0.5 & 0.5 & -0.9 & 0.3 & 0.5 & 0.5 & 0.5 & 0.5 & 447.19 \\
\hline 0.3 & 0.3 & 0.3 & 0.3 & 0.5 & 0.5 & -0.7 & 0.1 & 0.7 & 0.5 & 0.5 & 0.5 & 264.60 \\
\hline 0.3 & 0.3 & 0.3 & 0.3 & 0.5 & 0.5 & -0.7 & 0.5 & 0.5 & 0.5 & 0.5 & 0.5 & 447.19 \\
\hline 0.3 & 0.3 & 0.3 & 0.3 & 0.5 & 0.5 & -0.5 & 0.3 & 0.7 & 0.5 & 0.5 & 0.5 & 264.60 \\
\hline 0.3 & 0.3 & 0.3 & 0.3 & 0.5 & 0.5 & -0.5 & 0.7 & 0.5 & 0.5 & 0.5 & 0.5 & 447.19 \\
\hline 0.3 & 0.3 & 0.3 & 0.3 & 0.5 & 0.5 & -0.3 & 0.5 & 0.7 & 0.5 & 0.5 & 0.5 & 264.60 \\
\hline 0.3 & 0.3 & 0.3 & 0.3 & 0.5 & 0.5 & -0.1 & 0.7 & 0.7 & 0.5 & 0.5 & 0.5 & 264.60 \\
\hline 0.3 & 0.3 & 0.3 & 0.5 & 0.1 & 0.3 & -0.9 & 0.7 & 0.5 & 0.5 & 0.5 & 0.5 & 209.86 \\
\hline 0.3 & 0.3 & 0.3 & 0.5 & 0.1 & 0.3 & -0.9 & 0.7 & 0.7 & 0.5 & 0.5 & 0.5 & 232.88 \\
\hline 0.3 & 0.3 & 0.3 & 0.5 & 0.1 & 0.5 & -0.9 & 0.7 & 0.1 & 0.5 & 0.5 & 0.5 & 201.43 \\
\hline 0.3 & 0.3 & 0.3 & 0.5 & 0.1 & 0.5 & -0.9 & 0.7 & 0.3 & 0.5 & 0.5 & 0.5 & 239.27 \\
\hline 0.3 & 0.3 & 0.3 & 0.5 & 0.1 & 0.5 & -0.9 & 0.7 & 0.5 & 0.5 & 0.5 & 0.5 & 294.63 \\
\hline 0.3 & 0.3 & 0.3 & 0.5 & 0.1 & 0.5 & -0.9 & 0.7 & 0.7 & 0.5 & 0.5 & 0.5 & 383.32 \\
\hline 0.3 & 0.3 & 0.3 & 0.5 & 0.1 & 0.5 & -0.7 & 0.7 & 0.3 & 0.5 & 0.5 & 0.5 & 201.43 \\
\hline
\end{tabular}


Appendix

Table 3.1 (continued): Relative efficiency of the proposed ratio estimator with respect to the BBB model for different choice of parameters with $P=P_{1}=P_{2}=0.7$

\begin{tabular}{|c|c|c|c|c|c|c|c|c|c|c|c|c|}
\hline$C_{\gamma}$ & $C_{\gamma_{1}}$ & $C_{\gamma_{2}}$ & $C_{y}$ & $C x_{1}$ & $C x_{2}$ & $\rho_{y x_{1}}$ & $\rho_{y x_{2}}$ & $\rho_{x_{1} x_{2}}$ & $\theta$ & $\theta_{1}$ & $\theta_{2}$ & $\mathrm{RE}$ \\
\hline 0.3 & 0.3 & 0.3 & 0.5 & 0.1 & 0.5 & -0.7 & 0.7 & 0.5 & 0.5 & 0.5 & 0.5 & 239.27 \\
\hline 0.3 & 0.3 & 0.3 & 0.5 & 0.1 & 0.5 & -0.7 & 0.7 & 0.7 & 0.5 & 0.5 & 0.5 & 294.63 \\
\hline 0.3 & 0.3 & 0.3 & 0.5 & 0.1 & 0.5 & -0.5 & 0.7 & 0.5 & 0.5 & 0.5 & 0.5 & 201.43 \\
\hline 0.3 & 0.3 & 0.3 & 0.5 & 0.1 & 0.5 & -0.5 & 0.7 & 0.7 & 0.5 & 0.5 & 0.5 & 239.27 \\
\hline 0.3 & 0.3 & 0.3 & 0.5 & 0.1 & 0.5 & -0.3 & 0.7 & 0.7 & 0.5 & 0.5 & 0.5 & 201.43 \\
\hline 0.3 & 0.3 & 0.3 & 0.5 & 0.3 & 0.1 & -0.9 & 0.3 & 0.5 & 0.5 & 0.5 & 0.5 & 209.86 \\
\hline 0.3 & 0.3 & 0.3 & 0.5 & 0.3 & 0.1 & -0.9 & 0.3 & 0.7 & 0.5 & 0.5 & 0.5 & 232.88 \\
\hline 0.3 & 0.3 & 0.3 & 0.5 & 0.3 & 0.1 & -0.9 & 0.5 & 0.1 & 0.5 & 0.5 & 0.5 & 203.16 \\
\hline 0.3 & 0.3 & 0.3 & 0.5 & 0.3 & 0.1 & -0.9 & 0.5 & 0.3 & 0.5 & 0.5 & 0.5 & 224.67 \\
\hline 0.3 & 0.3 & 0.3 & 0.5 & 0.3 & 0.1 & -0.9 & 0.5 & 0.5 & 0.5 & 0.5 & 0.5 & 251.26 \\
\hline 0.3 & 0.3 & 0.3 & 0.5 & 0.3 & 0.1 & -0.9 & 0.5 & 0.7 & 0.5 & 0.5 & 0.5 & 285.00 \\
\hline 0.3 & 0.3 & 0.3 & 0.5 & 0.3 & 0.1 & -0.9 & 0.7 & 0.1 & 0.5 & 0.5 & 0.5 & 241.72 \\
\hline 0.3 & 0.3 & 0.3 & 0.5 & 0.3 & 0.1 & -0.9 & 0.7 & 0.3 & 0.5 & 0.5 & 0.5 & 272.79 \\
\hline 0.3 & 0.3 & 0.3 & 0.5 & 0.3 & 0.1 & -0.9 & 0.7 & 0.5 & 0.5 & 0.5 & 0.5 & 313.03 \\
\hline 0.3 & 0.3 & 0.3 & 0.5 & 0.3 & 0.1 & -0.9 & 0.7 & 0.7 & 0.5 & 0.5 & 0.5 & 367.18 \\
\hline 0.3 & 0.3 & 0.3 & 0.5 & 0.3 & 0.3 & -0.9 & 0.1 & 0.5 & 0.5 & 0.5 & 0.5 & 205.55 \\
\hline 0.3 & 0.3 & 0.3 & 0.5 & 0.3 & 0.3 & -0.9 & 0.1 & 0.7 & 0.5 & 0.5 & 0.5 & 289.74 \\
\hline 0.3 & 0.3 & 0.3 & 0.5 & 0.3 & 0.3 & -0.9 & 0.3 & 0.3 & 0.5 & 0.5 & 0.5 & 254.94 \\
\hline 0.3 & 0.3 & 0.3 & 0.5 & 0.3 & 0.3 & -0.9 & 0.3 & 0.5 & 0.5 & 0.5 & 0.5 & 398.56 \\
\hline 0.3 & 0.3 & 0.3 & 0.5 & 0.3 & 0.3 & -0.9 & 0.5 & 0.1 & 0.5 & 0.5 & 0.5 & 335.55 \\
\hline 0.3 & 0.3 & 0.3 & 0.5 & 0.3 & 0.3 & -0.7 & 0.3 & 0.5 & 0.5 & 0.5 & 0.5 & 205.55 \\
\hline 0.3 & 0.3 & 0.3 & 0.5 & 0.3 & 0.3 & -0.7 & 0.3 & 0.7 & 0.5 & 0.5 & 0.5 & 289.74 \\
\hline 0.3 & 0.3 & 0.3 & 0.5 & 0.3 & 0.3 & -0.7 & 0.5 & 0.3 & 0.5 & 0.5 & 0.5 & 254.94 \\
\hline 0.3 & 0.3 & 0.3 & 0.5 & 0.3 & 0.3 & -0.7 & 0.5 & 0.5 & 0.5 & 0.5 & 0.5 & 398.56 \\
\hline 0.3 & 0.3 & 0.3 & 0.5 & 0.3 & 0.3 & -0.7 & 0.7 & 0.1 & 0.5 & 0.5 & 0.5 & 335.55 \\
\hline 0.3 & 0.3 & 0.3 & 0.5 & 0.3 & 0.3 & -0.5 & 0.5 & 0.5 & 0.5 & 0.5 & 0.5 & 205.55 \\
\hline 0.3 & 0.3 & 0.3 & 0.5 & 0.3 & 0.3 & -0.5 & 0.5 & 0.7 & 0.5 & 0.5 & 0.5 & 289.74 \\
\hline 0.3 & 0.3 & 0.3 & 0.5 & 0.3 & 0.3 & -0.5 & 0.7 & 0.3 & 0.5 & 0.5 & 0.5 & 254.94 \\
\hline 0.3 & 0.3 & 0.3 & 0.5 & 0.3 & 0.3 & -0.5 & 0.7 & 0.5 & 0.5 & 0.5 & 0.5 & 398.56 \\
\hline 0.3 & 0.3 & 0.3 & 0.5 & 0.3 & 0.3 & -0.3 & 0.7 & 0.5 & 0.5 & 0.5 & 0.5 & 205.55 \\
\hline 0.3 & 0.3 & 0.3 & 0.5 & 0.3 & 0.3 & -0.3 & 0.7 & 0.7 & 0.5 & 0.5 & 0.5 & 289.74 \\
\hline 0.3 & 0.3 & 0.3 & 0.5 & 0.3 & 0.5 & -0.9 & 0.1 & 0.7 & 0.5 & 0.5 & 0.5 & 233.70 \\
\hline 0.3 & 0.3 & 0.3 & 0.5 & 0.3 & 0.5 & -0.9 & 0.3 & 0.5 & 0.5 & 0.5 & 0.5 & 369.21 \\
\hline 0.3 & 0.3 & 0.3 & 0.5 & 0.3 & 0.5 & -0.9 & 0.5 & 0.1 & 0.5 & 0.5 & 0.5 & 286.22 \\
\hline 0.3 & 0.3 & 0.3 & 0.5 & 0.3 & 0.5 & -0.7 & 0.3 & 0.7 & 0.5 & 0.5 & 0.5 & 369.21 \\
\hline 0.3 & 0.3 & 0.3 & 0.5 & 0.3 & 0.5 & -0.7 & 0.5 & 0.3 & 0.5 & 0.5 & 0.5 & 286.22 \\
\hline 0.3 & 0.3 & 0.3 & 0.5 & 0.3 & 0.5 & -0.5 & 0.5 & 0.5 & 0.5 & 0.5 & 0.5 & 286.22 \\
\hline 0.3 & 0.3 & 0.3 & 0.5 & 0.3 & 0.5 & -0.5 & 0.7 & 0.1 & 0.5 & 0.5 & 0.5 & 233.70 \\
\hline 0.3 & 0.3 & 0.3 & 0.5 & 0.3 & 0.5 & -0.3 & 0.5 & 0.7 & 0.5 & 0.5 & 0.5 & 286.22 \\
\hline 0.3 & 0.3 & 0.3 & 0.5 & 0.3 & 0.5 & -0.3 & 0.7 & 0.3 & 0.5 & 0.5 & 0.5 & 233.70 \\
\hline
\end{tabular}


ODUMADE \& SINGH

Appendix

Table 3.1 (continued): Relative efficiency of the proposed ratio estimator with respect to the BBB model for different choice of parameters with $P=P_{1}=P_{2}=0.7$

\begin{tabular}{|c|c|c|c|c|c|c|c|c|c|c|c|c|}
\hline$C_{\gamma}$ & $C_{\gamma_{1}}$ & $C_{\gamma_{2}}$ & $C_{y}$ & $C x_{1}$ & $C x_{2}$ & $\rho_{y x_{1}}$ & $\rho_{y x_{2}}$ & $\rho_{x_{1} x_{2}}$ & $\theta$ & $\theta_{1}$ & $\theta_{2}$ & RE \\
\hline 0.3 & 0.3 & 0.3 & 0.5 & $\overline{0.3}$ & 0.5 & $\overline{-0.1}$ & 0.7 & 0.5 & 0.5 & 0.5 & 0.5 & 233.70 \\
\hline 0.3 & 0.3 & 0.3 & 0.5 & 0.3 & 0.5 & 0.1 & 0.7 & 0.7 & 0.5 & 0.5 & 0.5 & 233.70 \\
\hline 0.3 & 0.3 & 0.3 & 0.5 & 0.5 & 0.1 & -0.9 & 0.1 & 0.1 & 0.5 & 0.5 & 0.5 & 239.27 \\
\hline 0.3 & 0.3 & 0.3 & 0.5 & 0.5 & 0.1 & -0.9 & 0.1 & 0.3 & 0.5 & 0.5 & 0.5 & 294.63 \\
\hline 0.3 & 0.3 & 0.3 & 0.5 & 0.5 & 0.1 & -0.9 & 0.1 & 0.5 & 0.5 & 0.5 & 0.5 & 383.32 \\
\hline 0.3 & 0.3 & 0.3 & 0.5 & 0.5 & 0.1 & -0.9 & 0.3 & 0.1 & 0.5 & 0.5 & 0.5 & 294.63 \\
\hline 0.3 & 0.3 & 0.3 & 0.5 & 0.5 & 0.1 & -0.9 & 0.3 & 0.3 & 0.5 & 0.5 & 0.5 & 383.32 \\
\hline 0.3 & 0.3 & 0.3 & 0.5 & 0.5 & 0.1 & -0.9 & 0.5 & 0.1 & 0.5 & 0.5 & 0.5 & 383.32 \\
\hline 0.3 & 0.3 & 0.3 & 0.5 & 0.5 & 0.1 & -0.7 & 0.3 & 0.7 & 0.5 & 0.5 & 0.5 & 201.43 \\
\hline 0.3 & 0.3 & 0.3 & 0.5 & 0.5 & 0.1 & -0.7 & 0.5 & 0.5 & 0.5 & 0.5 & 0.5 & 201.43 \\
\hline 0.3 & 0.3 & 0.3 & 0.5 & 0.5 & 0.1 & -0.7 & 0.5 & 0.7 & 0.5 & 0.5 & 0.5 & 239.27 \\
\hline 0.3 & 0.3 & 0.3 & 0.5 & 0.5 & 0.1 & -0.7 & 0.7 & 0.3 & 0.5 & 0.5 & 0.5 & 201.43 \\
\hline 0.3 & 0.3 & 0.3 & 0.5 & 0.5 & 0.1 & -0.7 & 0.7 & 0.5 & 0.5 & 0.5 & 0.5 & 239.27 \\
\hline 0.3 & 0.3 & 0.3 & 0.5 & 0.5 & 0.1 & -0.7 & 0.7 & 0.7 & 0.5 & 0.5 & 0.5 & 294.63 \\
\hline 0.3 & 0.3 & 0.3 & 0.5 & 0.5 & 0.3 & -0.9 & 0.1 & 0.3 & 0.5 & 0.5 & 0.5 & 369.21 \\
\hline 0.3 & 0.3 & 0.3 & 0.5 & 0.5 & 0.3 & -0.9 & 0.3 & 0.1 & 0.5 & 0.5 & 0.5 & 369.21 \\
\hline 0.3 & 0.3 & 0.3 & 0.5 & 0.5 & 0.3 & -0.7 & 0.1 & 0.5 & 0.5 & 0.5 & 0.5 & 233.70 \\
\hline 0.3 & 0.3 & 0.3 & 0.5 & 0.5 & 0.3 & -0.7 & 0.3 & 0.3 & 0.5 & 0.5 & 0.5 & 233.70 \\
\hline 0.3 & 0.3 & 0.3 & 0.5 & 0.5 & 0.3 & -0.7 & 0.5 & 0.1 & 0.5 & 0.5 & 0.5 & 233.70 \\
\hline 0.3 & 0.3 & 0.3 & 0.5 & 0.5 & 0.3 & -0.5 & 0.3 & 0.7 & 0.5 & 0.5 & 0.5 & 286.22 \\
\hline 0.3 & 0.3 & 0.3 & 0.5 & 0.5 & 0.3 & -0.5 & 0.5 & 0.5 & 0.5 & 0.5 & 0.5 & 286.22 \\
\hline 0.3 & 0.3 & 0.3 & 0.5 & 0.5 & 0.3 & -0.5 & 0.7 & 0.3 & 0.5 & 0.5 & 0.5 & 286.22 \\
\hline 0.3 & 0.3 & 0.3 & 0.5 & 0.5 & 0.3 & -0.3 & 0.7 & 0.7 & 0.5 & 0.5 & 0.5 & 369.21 \\
\hline 0.3 & 0.3 & 0.3 & 0.5 & 0.5 & 0.5 & -0.9 & 0.1 & 0.3 & 0.5 & 0.5 & 0.5 & 223.29 \\
\hline 0.3 & 0.3 & 0.3 & 0.5 & 0.5 & 0.5 & -0.9 & 0.3 & 0.1 & 0.5 & 0.5 & 0.5 & 223.29 \\
\hline 0.3 & 0.3 & 0.3 & 0.5 & 0.5 & 0.5 & -0.7 & 0.1 & 0.5 & 0.5 & 0.5 & 0.5 & 223.29 \\
\hline 0.3 & 0.3 & 0.3 & 0.5 & 0.5 & 0.5 & -0.7 & 0.3 & 0.3 & 0.5 & 0.5 & 0.5 & 223.29 \\
\hline 0.3 & 0.3 & 0.3 & 0.5 & 0.5 & 0.5 & -0.7 & 0.5 & 0.1 & 0.5 & 0.5 & 0.5 & 223.29 \\
\hline 0.3 & 0.3 & 0.3 & 0.5 & 0.5 & 0.5 & -0.5 & 0.1 & 0.7 & 0.5 & 0.5 & 0.5 & 223.29 \\
\hline 0.3 & 0.3 & 0.3 & 0.5 & 0.5 & 0.5 & -0.5 & 0.3 & 0.5 & 0.5 & 0.5 & 0.5 & 223.29 \\
\hline 0.3 & 0.3 & 0.3 & 0.5 & 0.5 & 0.5 & -0.5 & 0.5 & 0.3 & 0.5 & 0.5 & 0.5 & 223.29 \\
\hline 0.3 & 0.3 & 0.3 & 0.5 & 0.5 & 0.5 & -0.5 & 0.7 & 0.1 & 0.5 & 0.5 & 0.5 & 223.29 \\
\hline 0.3 & 0.3 & 0.3 & 0.5 & 0.5 & 0.5 & -0.3 & 0.3 & 0.7 & 0.5 & 0.5 & 0.5 & 223.29 \\
\hline 0.3 & 0.3 & 0.3 & 0.5 & 0.5 & 0.5 & -0.3 & 0.5 & 0.5 & 0.5 & 0.5 & 0.5 & 223.29 \\
\hline 0.3 & 0.3 & 0.3 & 0.5 & 0.5 & 0.5 & -0.3 & 0.7 & 0.3 & 0.5 & 0.5 & 0.5 & 223.29 \\
\hline 0.3 & 0.3 & 0.3 & 0.5 & 0.5 & 0.5 & -0.1 & 0.5 & 0.7 & 0.5 & 0.5 & 0.5 & 223.29 \\
\hline 0.3 & 0.3 & 0.3 & 0.5 & 0.5 & 0.5 & -0.1 & 0.7 & 0.5 & 0.5 & 0.5 & 0.5 & 223.29 \\
\hline 0.3 & 0.3 & 0.3 & 0.5 & 0.5 & 0.5 & 0.1 & 0.7 & 0.7 & 0.5 & 0.5 & 0.5 & 223.29 \\
\hline 0.5 & 0.5 & 0.5 & 0.3 & 0.3 & 0.3 & -0.9 & 0.5 & 0.7 & 0.5 & 0.5 & 0.5 & 285.02 \\
\hline 0.5 & 0.5 & 0.5 & 0.3 & 0.3 & 0.3 & -0.9 & 0.7 & 0.5 & 0.5 & 0.5 & 0.5 & 285.02 \\
\hline
\end{tabular}


Appendix

Table 3.1 (continued): Relative efficiency of the proposed ratio estimator with respect to the BBB model for different choice of parameters with $P=P_{1}=P_{2}=0.7$

\begin{tabular}{|c|c|c|c|c|c|c|c|c|c|c|c|c|}
\hline$C_{\gamma}$ & $C_{\gamma_{1}}$ & $C_{\gamma_{2}}$ & $C_{y}$ & $C x_{1}$ & $C x_{2}$ & $\rho_{y x_{1}}$ & $\rho_{y x_{2}}$ & $\rho_{x_{1} x_{2}}$ & $\theta$ & $\theta_{1}$ & $\theta_{2}$ & $\mathrm{RE}$ \\
\hline 0.5 & 0.5 & 0.5 & 0.3 & 0.3 & 0.3 & -0.7 & 0.7 & 0.7 & 0.5 & 0.5 & 0.5 & 285.02 \\
\hline 0.5 & 0.5 & 0.5 & 0.3 & 0.3 & 0.5 & -0.9 & 0.5 & 0.7 & 0.5 & 0.5 & 0.5 & 431.57 \\
\hline 0.5 & 0.5 & 0.5 & 0.3 & 0.3 & 0.5 & -0.9 & 0.7 & 0.5 & 0.5 & 0.5 & 0.5 & 431.57 \\
\hline 0.5 & 0.5 & 0.5 & 0.3 & 0.3 & 0.5 & -0.7 & 0.5 & 0.7 & 0.5 & 0.5 & 0.5 & 206.61 \\
\hline 0.5 & 0.5 & 0.5 & 0.3 & 0.3 & 0.5 & -0.7 & 0.7 & 0.5 & 0.5 & 0.5 & 0.5 & 206.61 \\
\hline 0.5 & 0.5 & 0.5 & 0.3 & 0.3 & 0.5 & -0.5 & 0.7 & 0.7 & 0.5 & 0.5 & 0.5 & 316.64 \\
\hline 0.5 & 0.5 & 0.5 & 0.3 & 0.5 & 0.3 & -0.9 & 0.1 & 0.7 & 0.5 & 0.5 & 0.5 & 250.05 \\
\hline 0.5 & 0.5 & 0.5 & 0.3 & 0.5 & 0.3 & -0.9 & 0.5 & 0.5 & 0.5 & 0.5 & 0.5 & 316.64 \\
\hline 0.5 & 0.5 & 0.5 & 0.3 & 0.5 & 0.3 & -0.9 & 0.7 & 0.3 & 0.5 & 0.5 & 0.5 & 206.61 \\
\hline 0.5 & 0.5 & 0.5 & 0.3 & 0.5 & 0.3 & -0.7 & 0.5 & 0.7 & 0.5 & 0.5 & 0.5 & 316.64 \\
\hline 0.5 & 0.5 & 0.5 & 0.3 & 0.5 & 0.3 & -0.7 & 0.7 & 0.5 & 0.5 & 0.5 & 0.5 & 206.61 \\
\hline 0.5 & 0.5 & 0.5 & 0.3 & 0.5 & 0.3 & -0.5 & 0.7 & 0.7 & 0.5 & 0.5 & 0.5 & 206.61 \\
\hline 0.5 & 0.5 & 0.5 & 0.3 & 0.5 & 0.5 & -0.9 & 0.1 & 0.7 & 0.5 & 0.5 & 0.5 & 445.03 \\
\hline 0.5 & 0.5 & 0.5 & 0.3 & 0.5 & 0.5 & -0.9 & 0.7 & 0.3 & 0.5 & 0.5 & 0.5 & 274.07 \\
\hline 0.5 & 0.5 & 0.5 & 0.3 & 0.5 & 0.5 & -0.7 & 0.3 & 0.7 & 0.5 & 0.5 & 0.5 & 445.03 \\
\hline 0.5 & 0.5 & 0.5 & 0.3 & 0.5 & 0.5 & -0.5 & 0.5 & 0.7 & 0.5 & 0.5 & 0.5 & 445.03 \\
\hline 0.5 & 0.5 & 0.5 & 0.3 & 0.5 & 0.5 & -0.3 & 0.7 & 0.7 & 0.5 & 0.5 & 0.5 & 445.03 \\
\hline 0.5 & 0.5 & 0.5 & 0.5 & 0.1 & 0.5 & -0.9 & 0.7 & 0.5 & 0.5 & 0.5 & 0.5 & 209.77 \\
\hline 0.5 & 0.5 & 0.5 & 0.5 & 0.1 & 0.5 & -0.9 & 0.7 & 0.7 & 0.5 & 0.5 & 0.5 & 248.42 \\
\hline 0.5 & 0.5 & 0.5 & 0.5 & 0.1 & 0.5 & -0.7 & 0.7 & 0.7 & 0.5 & 0.5 & 0.5 & 209.77 \\
\hline 0.5 & 0.5 & 0.5 & 0.5 & 0.3 & 0.1 & -0.9 & 0.5 & 0.7 & 0.5 & 0.5 & 0.5 & 208.15 \\
\hline 0.5 & 0.5 & 0.5 & 0.5 & 0.3 & 0.1 & -0.9 & 0.7 & 0.3 & 0.5 & 0.5 & 0.5 & 201.92 \\
\hline 0.5 & 0.5 & 0.5 & 0.5 & 0.3 & 0.1 & -0.9 & 0.7 & 0.5 & 0.5 & 0.5 & 0.5 & 221.85 \\
\hline 0.5 & 0.5 & 0.5 & 0.5 & 0.3 & 0.1 & -0.9 & 0.7 & 0.7 & 0.5 & 0.5 & 0.5 & 246.15 \\
\hline 0.5 & 0.5 & 0.5 & 0.5 & 0.3 & 0.3 & -0.9 & 0.1 & 0.7 & 0.5 & 0.5 & 0.5 & 208.96 \\
\hline 0.5 & 0.5 & 0.5 & 0.5 & 0.3 & 0.3 & -0.9 & 0.3 & 0.5 & 0.5 & 0.5 & 0.5 & 256.69 \\
\hline 0.5 & 0.5 & 0.5 & 0.5 & 0.3 & 0.3 & -0.9 & 0.3 & 0.7 & 0.5 & 0.5 & 0.5 & 390.50 \\
\hline 0.5 & 0.5 & 0.5 & 0.5 & 0.3 & 0.3 & -0.9 & 0.5 & 0.1 & 0.5 & 0.5 & 0.5 & 230.38 \\
\hline 0.5 & 0.5 & 0.5 & 0.5 & 0.3 & 0.3 & -0.9 & 0.5 & 0.3 & 0.5 & 0.5 & 0.5 & 332.69 \\
\hline 0.5 & 0.5 & 0.5 & 0.5 & 0.3 & 0.3 & -0.9 & 0.7 & 0.1 & 0.5 & 0.5 & 0.5 & 472.62 \\
\hline 0.5 & 0.5 & 0.5 & 0.5 & 0.3 & 0.3 & -0.7 & 0.3 & 0.7 & 0.5 & 0.5 & 0.5 & 208.96 \\
\hline 0.5 & 0.5 & 0.5 & 0.5 & 0.3 & 0.3 & -0.7 & 0.5 & 0.5 & 0.5 & 0.5 & 0.5 & 256.69 \\
\hline 0.5 & 0.5 & 0.5 & 0.5 & 0.3 & 0.3 & -0.7 & 0.5 & 0.7 & 0.5 & 0.5 & 0.5 & 390.50 \\
\hline 0.5 & 0.5 & 0.5 & 0.5 & 0.3 & 0.3 & -0.7 & 0.7 & 0.1 & 0.5 & 0.5 & 0.5 & 230.38 \\
\hline 0.5 & 0.5 & 0.5 & 0.5 & 0.3 & 0.3 & -0.7 & 0.7 & 0.3 & 0.5 & 0.5 & 0.5 & 332.69 \\
\hline 0.5 & 0.5 & 0.5 & 0.5 & 0.3 & 0.3 & -0.5 & 0.5 & 0.7 & 0.5 & 0.5 & 0.5 & 208.96 \\
\hline 0.5 & 0.5 & 0.5 & 0.5 & 0.3 & 0.3 & -0.5 & 0.7 & 0.5 & 0.5 & 0.5 & 0.5 & 256.69 \\
\hline 0.5 & 0.5 & 0.5 & 0.5 & 0.3 & 0.3 & -0.5 & 0.7 & 0.7 & 0.5 & 0.5 & 0.5 & 390.50 \\
\hline 0.5 & 0.5 & 0.5 & 0.5 & 0.3 & 0.3 & -0.3 & 0.7 & 0.7 & 0.5 & 0.5 & 0.5 & 208.96 \\
\hline 0.5 & 0.5 & 0.5 & 0.5 & 0.3 & 0.5 & -0.9 & 0.3 & 0.5 & 0.5 & 0.5 & 0.5 & 240.66 \\
\hline
\end{tabular}




\section{ODUMADE \& SINGH}

Appendix

Table 3.1 (continued): Relative efficiency of the proposed ratio estimator with respect to the BBB model for different choice of parameters with $P=P_{1}=P_{2}=0.7$

\begin{tabular}{|c|c|c|c|c|c|c|c|c|c|c|c|c|}
\hline$C_{\gamma}$ & $C_{\gamma_{1}}$ & $C_{\gamma_{2}}$ & $C_{y}$ & $C x_{1}$ & $C x_{2}$ & $\rho_{y x_{1}}$ & $\rho_{y x_{2}}$ & $\rho_{x_{1} x_{2}}$ & $\theta$ & $\theta_{1}$ & $\theta_{2}$ & $\mathrm{RE}$ \\
\hline 0.5 & 0.5 & 0.5 & 0.5 & 0.3 & 0.5 & -0.9 & 0.5 & 0.1 & 0.5 & 0.5 & 0.5 & 204.21 \\
\hline 0.5 & 0.5 & 0.5 & 0.5 & 0.3 & 0.5 & -0.9 & 0.5 & 0.3 & 0.5 & 0.5 & 0.5 & 374.24 \\
\hline 0.5 & 0.5 & 0.5 & 0.5 & 0.3 & 0.5 & -0.7 & 0.3 & 0.7 & 0.5 & 0.5 & 0.5 & 240.66 \\
\hline 0.5 & 0.5 & 0.5 & 0.5 & 0.3 & 0.5 & -0.7 & 0.5 & 0.3 & 0.5 & 0.5 & 0.5 & 204.21 \\
\hline 0.5 & 0.5 & 0.5 & 0.5 & 0.3 & 0.5 & -0.7 & 0.5 & 0.5 & 0.5 & 0.5 & 0.5 & 374.24 \\
\hline 0.5 & 0.5 & 0.5 & 0.5 & 0.3 & 0.5 & -0.7 & 0.7 & 0.1 & 0.5 & 0.5 & 0.5 & 292.94 \\
\hline 0.5 & 0.5 & 0.5 & 0.5 & 0.3 & 0.5 & -0.5 & 0.5 & 0.5 & 0.5 & 0.5 & 0.5 & 204.21 \\
\hline 0.5 & 0.5 & 0.5 & 0.5 & 0.3 & 0.5 & -0.5 & 0.5 & 0.7 & 0.5 & 0.5 & 0.5 & 374.24 \\
\hline 0.5 & 0.5 & 0.5 & 0.5 & 0.3 & 0.5 & -0.5 & 0.7 & 0.3 & 0.5 & 0.5 & 0.5 & 292.94 \\
\hline 0.5 & 0.5 & 0.5 & 0.5 & 0.3 & 0.5 & -0.3 & 0.5 & 0.7 & 0.5 & 0.5 & 0.5 & 204.21 \\
\hline 0.5 & 0.5 & 0.5 & 0.5 & 0.3 & 0.5 & -0.3 & 0.7 & 0.5 & 0.5 & 0.5 & 0.5 & 292.94 \\
\hline 0.5 & 0.5 & 0.5 & 0.5 & 0.3 & 0.5 & -0.1 & 0.7 & 0.7 & 0.5 & 0.5 & 0.5 & 292.94 \\
\hline 0.5 & 0.5 & 0.5 & 0.5 & 0.5 & 0.1 & -0.9 & 0.1 & 0.3 & 0.5 & 0.5 & 0.5 & 209.77 \\
\hline 0.5 & 0.5 & 0.5 & 0.5 & 0.5 & 0.1 & -0.9 & 0.1 & 0.5 & 0.5 & 0.5 & 0.5 & 248.42 \\
\hline 0.5 & 0.5 & 0.5 & 0.5 & 0.5 & 0.1 & -0.9 & 0.1 & 0.7 & 0.5 & 0.5 & 0.5 & 304.52 \\
\hline 0.5 & 0.5 & 0.5 & 0.5 & 0.5 & 0.1 & -0.9 & 0.3 & 0.1 & 0.5 & 0.5 & 0.5 & 209.77 \\
\hline 0.5 & 0.5 & 0.5 & 0.5 & 0.5 & 0.1 & -0.9 & 0.3 & 0.3 & 0.5 & 0.5 & 0.5 & 248.42 \\
\hline 0.5 & 0.5 & 0.5 & 0.5 & 0.5 & 0.1 & -0.9 & 0.3 & 0.5 & 0.5 & 0.5 & 0.5 & 304.52 \\
\hline 0.5 & 0.5 & 0.5 & 0.5 & 0.5 & 0.1 & -0.9 & 0.3 & 0.7 & 0.5 & 0.5 & 0.5 & 393.35 \\
\hline 0.5 & 0.5 & 0.5 & 0.5 & 0.5 & 0.1 & -0.9 & 0.5 & 0.1 & 0.5 & 0.5 & 0.5 & 248.42 \\
\hline 0.5 & 0.5 & 0.5 & 0.5 & 0.5 & 0.1 & -0.9 & 0.5 & 0.3 & 0.5 & 0.5 & 0.5 & 304.52 \\
\hline 0.5 & 0.5 & 0.5 & 0.5 & 0.5 & 0.1 & -0.9 & 0.5 & 0.5 & 0.5 & 0.5 & 0.5 & 393.35 \\
\hline 0.5 & 0.5 & 0.5 & 0.5 & 0.5 & 0.1 & -0.9 & 0.7 & 0.1 & 0.5 & 0.5 & 0.5 & 304.52 \\
\hline 0.5 & 0.5 & 0.5 & 0.5 & 0.5 & 0.1 & -0.9 & 0.7 & 0.3 & 0.5 & 0.5 & 0.5 & 393.35 \\
\hline 0.5 & 0.5 & 0.5 & 0.5 & 0.5 & 0.1 & -0.7 & 0.7 & 0.7 & 0.5 & 0.5 & 0.5 & 209.77 \\
\hline 0.5 & 0.5 & 0.5 & 0.5 & 0.5 & 0.3 & -0.9 & 0.1 & 0.3 & 0.5 & 0.5 & 0.5 & 240.66 \\
\hline 0.5 & 0.5 & 0.5 & 0.5 & 0.5 & 0.3 & -0.9 & 0.3 & 0.1 & 0.5 & 0.5 & 0.5 & 240.66 \\
\hline 0.5 & 0.5 & 0.5 & 0.5 & 0.5 & 0.3 & -0.7 & 0.1 & 0.7 & 0.5 & 0.5 & 0.5 & 292.94 \\
\hline 0.5 & 0.5 & 0.5 & 0.5 & 0.5 & 0.3 & -0.7 & 0.3 & 0.5 & 0.5 & 0.5 & 0.5 & 292.94 \\
\hline 0.5 & 0.5 & 0.5 & 0.5 & 0.5 & 0.3 & -0.7 & 0.5 & 0.3 & 0.5 & 0.5 & 0.5 & 292.94 \\
\hline 0.5 & 0.5 & 0.5 & 0.5 & 0.5 & 0.3 & -0.7 & 0.7 & 0.1 & 0.5 & 0.5 & 0.5 & 292.94 \\
\hline 0.5 & 0.5 & 0.5 & 0.5 & 0.5 & 0.3 & -0.5 & 0.3 & 0.7 & 0.5 & 0.5 & 0.5 & 204.21 \\
\hline 0.5 & 0.5 & 0.5 & 0.5 & 0.5 & 0.3 & -0.5 & 0.5 & 0.5 & 0.5 & 0.5 & 0.5 & 204.21 \\
\hline 0.5 & 0.5 & 0.5 & 0.5 & 0.5 & 0.3 & -0.5 & 0.5 & 0.7 & 0.5 & 0.5 & 0.5 & 374.24 \\
\hline 0.5 & 0.5 & 0.5 & 0.5 & 0.5 & 0.3 & -0.5 & 0.7 & 0.3 & 0.5 & 0.5 & 0.5 & 204.21 \\
\hline 0.5 & 0.5 & 0.5 & 0.5 & 0.5 & 0.3 & -0.5 & 0.7 & 0.5 & 0.5 & 0.5 & 0.5 & 374.24 \\
\hline 0.5 & 0.5 & 0.5 & 0.5 & 0.5 & 0.3 & -0.3 & 0.7 & 0.7 & 0.5 & 0.5 & 0.5 & 240.66 \\
\hline 0.5 & 0.5 & 0.5 & 0.5 & 0.5 & 0.5 & -0.9 & 0.1 & 0.5 & 0.5 & 0.5 & 0.5 & 456.61 \\
\hline 0.5 & 0.5 & 0.5 & 0.5 & 0.5 & 0.5 & -0.9 & 0.3 & 0.3 & 0.5 & 0.5 & 0.5 & 456.61 \\
\hline 0.5 & 0.5 & 0.5 & 0.5 & 0.5 & 0.5 & -0.9 & 0.5 & 0.1 & 0.5 & 0.5 & 0.5 & 456.61 \\
\hline
\end{tabular}


Appendix

Table 3.1 (continued): Relative efficiency of the proposed ratio estimator with respect to the BBB model for different choice of parameters with $P=P_{1}=P_{2}=0.7$

\begin{tabular}{|c|c|c|c|c|c|c|c|c|c|c|c|c|}
\hline$C_{\gamma}$ & $C_{\gamma_{1}}$ & $C_{\gamma_{2}}$ & $C_{y}$ & $C x_{1}$ & $C x_{2}$ & $\rho_{y x_{1}}$ & $\rho_{y x_{2}}$ & $\rho_{x_{1} x_{2}}$ & $\theta$ & $\theta_{1}$ & $\theta_{2}$ & RE \\
\hline 0.5 & 0.5 & 0.5 & 0.5 & 0.5 & 0.5 & -0.7 & 0.1 & 0.7 & 0.5 & 0.5 & 0.5 & 456.61 \\
\hline 0.5 & 0.5 & 0.5 & 0.5 & 0.5 & 0.5 & -0.7 & 0.3 & 0.5 & 0.5 & 0.5 & 0.5 & 456.61 \\
\hline 0.5 & 0.5 & 0.5 & 0.5 & 0.5 & 0.5 & -0.7 & 0.5 & 0.3 & 0.5 & 0.5 & 0.5 & 456.61 \\
\hline 0.5 & 0.5 & 0.5 & 0.5 & 0.5 & 0.5 & -0.7 & 0.7 & 0.1 & 0.5 & 0.5 & 0.5 & 456.61 \\
\hline 0.5 & 0.5 & 0.5 & 0.5 & 0.5 & 0.5 & -0.5 & 0.3 & 0.7 & 0.5 & 0.5 & 0.5 & 456.61 \\
\hline 0.5 & 0.5 & 0.5 & 0.5 & 0.5 & 0.5 & -0.5 & 0.5 & 0.5 & 0.5 & 0.5 & 0.5 & 456.61 \\
\hline 0.5 & 0.5 & 0.5 & 0.5 & 0.5 & 0.5 & -0.5 & 0.7 & 0.3 & 0.5 & 0.5 & 0.5 & 456.61 \\
\hline 0.5 & 0.5 & 0.5 & 0.5 & 0.5 & 0.5 & -0.3 & 0.5 & 0.7 & 0.5 & 0.5 & 0.5 & 456.61 \\
\hline 0.5 & 0.5 & 0.5 & 0.5 & 0.5 & 0.5 & -0.3 & 0.7 & 0.5 & 0.5 & 0.5 & 0.5 & 456.61 \\
\hline 0.5 & 0.5 & 0.5 & 0.5 & 0.5 & 0.5 & -0.1 & 0.7 & 0.7 & 0.5 & 0.5 & 0.5 & 456.61 \\
\hline 0.1 & 0.1 & 0.1 & 0.1 & 0.1 & 0.1 & -0.9 & 0.1 & 0.7 & 1.0 & 1.0 & 1.0 & 255.99 \\
\hline 0.1 & 0.1 & 0.1 & 0.1 & 0.1 & 0.1 & -0.9 & 0.3 & 0.5 & 1.0 & 1.0 & 1.0 & 255.99 \\
\hline 0.1 & 0.1 & 0.1 & 0.1 & 0.1 & 0.1 & -0.9 & 0.5 & 0.3 & 1.0 & 1.0 & 1.0 & 255.99 \\
\hline 0.1 & 0.1 & 0.1 & 0.1 & 0.1 & 0.1 & -0.9 & 0.7 & 0.1 & 1.0 & 1.0 & 1.0 & 255.99 \\
\hline 0.1 & 0.1 & 0.1 & 0.1 & 0.1 & 0.1 & -0.7 & 0.3 & 0.7 & 1.0 & 1.0 & 1.0 & 255.99 \\
\hline 0.1 & 0.1 & 0.1 & 0.1 & 0.1 & 0.1 & -0.7 & 0.5 & 0.5 & 1.0 & 1.0 & 1.0 & 255.99 \\
\hline 0.1 & 0.1 & 0.1 & 0.1 & 0.1 & 0.1 & -0.7 & 0.7 & 0.3 & 1.0 & 1.0 & 1.0 & 255.99 \\
\hline 0.1 & 0.1 & 0.1 & 0.1 & 0.1 & 0.1 & -0.5 & 0.5 & 0.7 & 1.0 & 1.0 & 1.0 & 255.99 \\
\hline 0.1 & 0.1 & 0.1 & 0.1 & 0.1 & 0.1 & -0.5 & 0.7 & 0.5 & 1.0 & 1.0 & 1.0 & 255.99 \\
\hline 0.1 & 0.1 & 0.1 & 0.1 & 0.1 & 0.1 & -0.3 & 0.7 & 0.7 & 1.0 & 1.0 & 1.0 & 255.99 \\
\hline 0.1 & 0.1 & 0.1 & 0.3 & 0.1 & 0.1 & -0.9 & 0.1 & 0.7 & 1.0 & 1.0 & 1.0 & 205.76 \\
\hline 0.1 & 0.1 & 0.1 & 0.3 & 0.1 & 0.1 & -0.9 & 0.3 & 0.1 & 1.0 & 1.0 & 1.0 & 205.76 \\
\hline 0.1 & 0.1 & 0.1 & 0.3 & 0.1 & 0.1 & -0.9 & 0.3 & 0.3 & 1.0 & 1.0 & 1.0 & 225.67 \\
\hline 0.1 & 0.1 & 0.1 & 0.3 & 0.1 & 0.1 & -0.9 & 0.3 & 0.5 & 1.0 & 1.0 & 1.0 & 249.85 \\
\hline 0.1 & 0.1 & 0.1 & 0.3 & 0.1 & 0.1 & -0.9 & 0.3 & 0.7 & 1.0 & 1.0 & 1.0 & 279.84 \\
\hline 0.1 & 0.1 & 0.1 & 0.3 & 0.1 & 0.1 & -0.9 & 0.5 & 0.1 & 1.0 & 1.0 & 1.0 & 279.84 \\
\hline 0.1 & 0.1 & 0.1 & 0.3 & 0.1 & 0.1 & -0.9 & 0.5 & 0.3 & 1.0 & 1.0 & 1.0 & 318.00 \\
\hline 0.1 & 0.1 & 0.1 & 0.3 & 0.1 & 0.1 & -0.9 & 0.5 & 0.5 & 1.0 & 1.0 & 1.0 & 368.22 \\
\hline 0.1 & 0.1 & 0.1 & 0.3 & 0.1 & 0.1 & -0.9 & 0.5 & 0.7 & 1.0 & 1.0 & 1.0 & 437.27 \\
\hline 0.1 & 0.1 & 0.1 & 0.3 & 0.1 & 0.1 & -0.9 & 0.7 & 0.1 & 1.0 & 1.0 & 1.0 & 437.27 \\
\hline 0.1 & 0.1 & 0.1 & 0.3 & 0.1 & 0.1 & -0.7 & 0.3 & 0.7 & 1.0 & 1.0 & 1.0 & 205.76 \\
\hline 0.1 & 0.1 & 0.1 & 0.3 & 0.1 & 0.1 & -0.7 & 0.5 & 0.1 & 1.0 & 1.0 & 1.0 & 205.76 \\
\hline 0.1 & 0.1 & 0.1 & 0.3 & 0.1 & 0.1 & -0.7 & 0.5 & 0.3 & 1.0 & 1.0 & 1.0 & 225.67 \\
\hline 0.1 & 0.1 & 0.1 & 0.3 & 0.1 & 0.1 & -0.7 & 0.5 & 0.5 & 1.0 & 1.0 & 1.0 & 249.85 \\
\hline 0.1 & 0.1 & 0.1 & 0.3 & 0.1 & 0.1 & -0.7 & 0.5 & 0.7 & 1.0 & 1.0 & 1.0 & 279.84 \\
\hline 0.1 & 0.1 & 0.1 & 0.3 & 0.1 & 0.1 & -0.7 & 0.7 & 0.1 & 1.0 & 1.0 & 1.0 & 279.84 \\
\hline 0.1 & 0.1 & 0.1 & 0.3 & 0.1 & 0.1 & -0.7 & 0.7 & 0.3 & 1.0 & 1.0 & 1.0 & 318.00 \\
\hline 0.1 & 0.1 & 0.1 & 0.3 & 0.1 & 0.1 & -0.7 & 0.7 & 0.5 & 1.0 & 1.0 & 1.0 & 368.22 \\
\hline 0.1 & 0.1 & 0.1 & 0.3 & 0.1 & 0.1 & -0.7 & 0.7 & 0.7 & 1.0 & 1.0 & 1.0 & 437.27 \\
\hline 0.1 & 0.1 & 0.1 & 0.3 & 0.1 & 0.1 & -0.5 & 0.5 & 0.7 & 1.0 & 1.0 & 1.0 & 205.76 \\
\hline
\end{tabular}




\section{ODUMADE \& SINGH}

Appendix

Table 3.1 (continued): Relative efficiency of the proposed ratio estimator with respect to the BBB model for different choice of parameters with $P=P_{1}=P_{2}=0.7$

\begin{tabular}{|c|c|c|c|c|c|c|c|c|c|c|c|c|}
\hline$C_{\gamma}$ & $C_{\gamma_{1}}$ & $C_{\gamma_{2}}$ & $C_{y}$ & $C x_{1}$ & $C x_{2}$ & $\rho_{y x_{1}}$ & $\rho_{y x_{2}}$ & $\rho_{x_{1} x_{2}}$ & $\theta$ & $\theta_{1}$ & $\theta_{2}$ & RE \\
\hline 0.1 & 0.1 & 0.1 & 0.3 & 0.1 & 0.1 & -0.5 & 0.7 & 0.1 & 1.0 & 1.0 & 1.0 & 205.76 \\
\hline 0.1 & 0.1 & 0.1 & 0.3 & 0.1 & 0.1 & -0.5 & 0.7 & 0.3 & 1.0 & 1.0 & 1.0 & 225.67 \\
\hline 0.1 & 0.1 & 0.1 & 0.3 & 0.1 & 0.1 & -0.5 & 0.7 & 0.5 & 1.0 & 1.0 & 1.0 & 249.85 \\
\hline 0.1 & 0.1 & 0.1 & 0.3 & 0.1 & 0.1 & -0.5 & 0.7 & 0.7 & 1.0 & 1.0 & 1.0 & 279.84 \\
\hline 0.1 & 0.1 & 0.1 & 0.3 & 0.1 & 0.1 & -0.3 & 0.7 & 0.7 & 1.0 & 1.0 & 1.0 & 205.76 \\
\hline 0.1 & 0.1 & 0.1 & 0.3 & 0.1 & 0.3 & -0.9 & 0.5 & 0.3 & 1.0 & 1.0 & 1.0 & 248.26 \\
\hline 0.1 & 0.1 & 0.1 & 0.3 & 0.1 & 0.3 & -0.9 & 0.5 & 0.5 & 1.0 & 1.0 & 1.0 & 364.76 \\
\hline 0.1 & 0.1 & 0.1 & 0.3 & 0.1 & 0.3 & -0.7 & 0.5 & 0.5 & 1.0 & 1.0 & 1.0 & 248.26 \\
\hline 0.1 & 0.1 & 0.1 & 0.3 & 0.1 & 0.3 & -0.7 & 0.5 & 0.7 & 1.0 & 1.0 & 1.0 & 364.76 \\
\hline 0.1 & 0.1 & 0.1 & 0.3 & 0.1 & 0.3 & -0.7 & 0.7 & 0.1 & 1.0 & 1.0 & 1.0 & 364.76 \\
\hline 0.1 & 0.1 & 0.1 & 0.3 & 0.1 & 0.3 & -0.5 & 0.5 & 0.7 & 1.0 & 1.0 & 1.0 & 248.26 \\
\hline 0.1 & 0.1 & 0.1 & 0.3 & 0.1 & 0.3 & -0.5 & 0.7 & 0.1 & 1.0 & 1.0 & 1.0 & 248.26 \\
\hline 0.1 & 0.1 & 0.1 & 0.3 & 0.1 & 0.3 & -0.5 & 0.7 & 0.3 & 1.0 & 1.0 & 1.0 & 364.76 \\
\hline 0.1 & 0.1 & 0.1 & 0.3 & 0.1 & 0.3 & -0.3 & 0.7 & 0.3 & 1.0 & 1.0 & 1.0 & 248.26 \\
\hline 0.1 & 0.1 & 0.1 & 0.3 & 0.1 & 0.3 & -0.3 & 0.7 & 0.5 & 1.0 & 1.0 & 1.0 & 364.76 \\
\hline 0.1 & 0.1 & 0.1 & 0.3 & 0.1 & 0.3 & -0.1 & 0.7 & 0.5 & 1.0 & 1.0 & 1.0 & 248.26 \\
\hline 0.1 & 0.1 & 0.1 & 0.3 & 0.1 & 0.3 & -0.1 & 0.7 & 0.7 & 1.0 & 1.0 & 1.0 & 364.76 \\
\hline 0.1 & 0.1 & 0.1 & 0.3 & 0.1 & 0.3 & 0.1 & 0.7 & 0.7 & 1.0 & 1.0 & 1.0 & 248.26 \\
\hline 0.1 & 0.1 & 0.1 & 0.3 & 0.1 & 0.5 & -0.9 & 0.7 & 0.5 & 1.0 & 1.0 & 1.0 & 202.54 \\
\hline 0.1 & 0.1 & 0.1 & 0.3 & 0.1 & 0.5 & -0.9 & 0.7 & 0.7 & 1.0 & 1.0 & 1.0 & 358.04 \\
\hline 0.1 & 0.1 & 0.1 & 0.3 & 0.1 & 0.5 & -0.7 & 0.7 & 0.7 & 1.0 & 1.0 & 1.0 & 245.12 \\
\hline 0.1 & 0.1 & 0.1 & 0.3 & 0.3 & 0.1 & -0.9 & 0.1 & 0.1 & 1.0 & 1.0 & 1.0 & 364.76 \\
\hline 0.1 & 0.1 & 0.1 & 0.3 & 0.3 & 0.1 & -0.7 & 0.1 & 0.5 & 1.0 & 1.0 & 1.0 & 248.26 \\
\hline 0.1 & 0.1 & 0.1 & 0.3 & 0.3 & 0.1 & -0.7 & 0.1 & 0.7 & 1.0 & 1.0 & 1.0 & 364.76 \\
\hline 0.1 & 0.1 & 0.1 & 0.3 & 0.3 & 0.1 & -0.7 & 0.3 & 0.3 & 1.0 & 1.0 & 1.0 & 248.26 \\
\hline 0.1 & 0.1 & 0.1 & 0.3 & 0.3 & 0.1 & -0.7 & 0.3 & 0.5 & 1.0 & 1.0 & 1.0 & 364.76 \\
\hline 0.1 & 0.1 & 0.1 & 0.3 & 0.3 & 0.1 & -0.7 & 0.5 & 0.1 & 1.0 & 1.0 & 1.0 & 248.26 \\
\hline 0.1 & 0.1 & 0.1 & 0.3 & 0.3 & 0.1 & -0.7 & 0.5 & 0.3 & 1.0 & 1.0 & 1.0 & 364.76 \\
\hline 0.1 & 0.1 & 0.1 & 0.3 & 0.3 & 0.1 & -0.7 & 0.7 & 0.1 & 1.0 & 1.0 & 1.0 & 364.76 \\
\hline 0.1 & 0.1 & 0.1 & 0.3 & 0.3 & 0.1 & -0.5 & 0.5 & 0.7 & 1.0 & 1.0 & 1.0 & 248.26 \\
\hline 0.1 & 0.1 & 0.1 & 0.3 & 0.3 & 0.1 & -0.5 & 0.7 & 0.5 & 1.0 & 1.0 & 1.0 & 248.26 \\
\hline 0.1 & 0.1 & 0.1 & 0.3 & 0.3 & 0.1 & -0.5 & 0.7 & 0.7 & 1.0 & 1.0 & 1.0 & 364.76 \\
\hline 0.1 & 0.1 & 0.1 & 0.3 & 0.3 & 0.3 & -0.9 & 0.1 & 0.3 & 1.0 & 1.0 & 1.0 & 203.60 \\
\hline 0.1 & 0.1 & 0.1 & 0.3 & 0.3 & 0.3 & -0.9 & 0.3 & 0.1 & 1.0 & 1.0 & 1.0 & 203.60 \\
\hline 0.1 & 0.1 & 0.1 & 0.3 & 0.3 & 0.3 & -0.7 & 0.1 & 0.5 & 1.0 & 1.0 & 1.0 & 203.60 \\
\hline 0.1 & 0.1 & 0.1 & 0.3 & 0.3 & 0.3 & -0.7 & 0.3 & 0.3 & 1.0 & 1.0 & 1.0 & 203.60 \\
\hline 0.1 & 0.1 & 0.1 & 0.3 & 0.3 & 0.3 & -0.7 & 0.5 & 0.1 & 1.0 & 1.0 & 1.0 & 203.60 \\
\hline 0.1 & 0.1 & 0.1 & 0.3 & 0.3 & 0.3 & -0.5 & 0.1 & 0.7 & 1.0 & 1.0 & 1.0 & 203.60 \\
\hline 0.1 & 0.1 & 0.1 & 0.3 & 0.3 & 0.3 & -0.5 & 0.3 & 0.5 & 1.0 & 1.0 & 1.0 & 203.60 \\
\hline 0.1 & 0.1 & 0.1 & 0.3 & 0.3 & 0.3 & -0.5 & 0.5 & 0.3 & 1.0 & 1.0 & 1.0 & 203.60 \\
\hline
\end{tabular}


Appendix

Table 3.1 (continued): Relative efficiency of the proposed ratio estimator with respect to the BBB model for different choice of parameters with $P=P_{1}=P_{2}=0.7$

\begin{tabular}{|c|c|c|c|c|c|c|c|c|c|c|c|c|}
\hline$C_{\gamma}$ & $C_{\gamma_{1}}$ & $C_{\gamma_{2}}$ & $C_{y}$ & $C x_{1}$ & $C x_{2}$ & $\rho_{y x_{1}}$ & $\rho_{y x_{2}}$ & $\rho_{x_{1} x_{2}}$ & $\theta$ & $\theta_{1}$ & $\theta_{2}$ & $\mathrm{RE}$ \\
\hline 0.1 & 0.1 & 0.1 & 0.3 & 0.3 & 0.3 & -0.5 & 0.7 & 0.1 & 1.0 & 1.0 & 1.0 & 203.60 \\
\hline 0.1 & 0.1 & 0.1 & 0.3 & 0.3 & 0.3 & -0.3 & 0.3 & 0.7 & 1.0 & 1.0 & 1.0 & 203.60 \\
\hline 0.1 & 0.1 & 0.1 & 0.3 & 0.3 & 0.3 & -0.3 & 0.5 & 0.5 & 1.0 & 1.0 & 1.0 & 203.60 \\
\hline 0.1 & 0.1 & 0.1 & 0.3 & 0.3 & 0.3 & -0.3 & 0.7 & 0.3 & 1.0 & 1.0 & 1.0 & 203.60 \\
\hline 0.1 & 0.1 & 0.1 & 0.3 & 0.3 & 0.3 & -0.1 & 0.5 & 0.7 & 1.0 & 1.0 & 1.0 & 203.60 \\
\hline 0.1 & 0.1 & 0.1 & 0.3 & 0.3 & 0.3 & -0.1 & 0.7 & 0.5 & 1.0 & 1.0 & 1.0 & 203.60 \\
\hline 0.1 & 0.1 & 0.1 & 0.3 & 0.3 & 0.3 & 0.1 & 0.7 & 0.7 & 1.0 & 1.0 & 1.0 & 203.60 \\
\hline 0.1 & 0.1 & 0.1 & 0.3 & 0.3 & 0.5 & -0.9 & 0.1 & 0.7 & 1.0 & 1.0 & 1.0 & 243.59 \\
\hline 0.1 & 0.1 & 0.1 & 0.3 & 0.3 & 0.5 & -0.9 & 0.3 & 0.5 & 1.0 & 1.0 & 1.0 & 243.59 \\
\hline 0.1 & 0.1 & 0.1 & 0.3 & 0.3 & 0.5 & -0.9 & 0.5 & 0.3 & 1.0 & 1.0 & 1.0 & 243.59 \\
\hline 0.1 & 0.1 & 0.1 & 0.3 & 0.3 & 0.5 & -0.9 & 0.7 & 0.1 & 1.0 & 1.0 & 1.0 & 243.59 \\
\hline 0.1 & 0.1 & 0.1 & 0.3 & 0.3 & 0.5 & -0.3 & 0.5 & 0.7 & 1.0 & 1.0 & 1.0 & 354.77 \\
\hline 0.1 & 0.1 & 0.1 & 0.3 & 0.3 & 0.5 & -0.3 & 0.7 & 0.5 & 1.0 & 1.0 & 1.0 & 354.77 \\
\hline 0.1 & 0.1 & 0.1 & 0.3 & 0.3 & 0.5 & 0.1 & 0.7 & 0.7 & 1.0 & 1.0 & 1.0 & 243.59 \\
\hline 0.1 & 0.1 & 0.1 & 0.3 & 0.5 & 0.1 & -0.9 & 0.1 & 0.5 & 1.0 & 1.0 & 1.0 & 273.92 \\
\hline 0.1 & 0.1 & 0.1 & 0.3 & 0.5 & 0.1 & -0.9 & 0.3 & 0.3 & 1.0 & 1.0 & 1.0 & 221.81 \\
\hline 0.1 & 0.1 & 0.1 & 0.3 & 0.5 & 0.1 & -0.9 & 0.3 & 0.5 & 1.0 & 1.0 & 1.0 & 422.99 \\
\hline 0.1 & 0.1 & 0.1 & 0.3 & 0.5 & 0.1 & -0.9 & 0.5 & 0.3 & 1.0 & 1.0 & 1.0 & 310.38 \\
\hline 0.1 & 0.1 & 0.1 & 0.3 & 0.5 & 0.1 & -0.9 & 0.7 & 0.1 & 1.0 & 1.0 & 1.0 & 245.12 \\
\hline 0.1 & 0.1 & 0.1 & 0.3 & 0.5 & 0.1 & -0.7 & 0.7 & 0.7 & 1.0 & 1.0 & 1.0 & 245.12 \\
\hline 0.1 & 0.1 & 0.1 & 0.3 & 0.5 & 0.3 & -0.9 & 0.3 & 0.3 & 1.0 & 1.0 & 1.0 & 354.77 \\
\hline 0.1 & 0.1 & 0.1 & 0.3 & 0.5 & 0.3 & -0.7 & 0.3 & 0.5 & 1.0 & 1.0 & 1.0 & 354.77 \\
\hline 0.1 & 0.1 & 0.1 & 0.3 & 0.5 & 0.3 & -0.5 & 0.3 & 0.7 & 1.0 & 1.0 & 1.0 & 354.77 \\
\hline 0.1 & 0.1 & 0.1 & 0.3 & 0.5 & 0.5 & -0.9 & 0.5 & 0.3 & 1.0 & 1.0 & 1.0 & 303.12 \\
\hline 0.1 & 0.1 & 0.1 & 0.3 & 0.5 & 0.5 & -0.7 & 0.7 & 0.3 & 1.0 & 1.0 & 1.0 & 303.12 \\
\hline 0.1 & 0.1 & 0.1 & 0.5 & 0.1 & 0.1 & -0.9 & 0.5 & 0.7 & 1.0 & 1.0 & 1.0 & 201.69 \\
\hline 0.1 & 0.1 & 0.1 & 0.5 & 0.1 & 0.1 & -0.9 & 0.7 & 0.1 & 1.0 & 1.0 & 1.0 & 215.39 \\
\hline 0.1 & 0.1 & 0.1 & 0.5 & 0.1 & 0.1 & -0.9 & 0.7 & 0.3 & 1.0 & 1.0 & 1.0 & 222.96 \\
\hline 0.1 & 0.1 & 0.1 & 0.5 & 0.1 & 0.1 & -0.9 & 0.7 & 0.5 & 1.0 & 1.0 & 1.0 & 231.08 \\
\hline 0.1 & 0.1 & 0.1 & 0.5 & 0.1 & 0.1 & -0.9 & 0.7 & 0.7 & 1.0 & 1.0 & 1.0 & 239.82 \\
\hline 0.1 & 0.1 & 0.1 & 0.5 & 0.1 & 0.1 & -0.7 & 0.7 & 0.7 & 1.0 & 1.0 & 1.0 & 201.69 \\
\hline 0.1 & 0.1 & 0.1 & 0.5 & 0.1 & 0.3 & -0.9 & 0.5 & 0.1 & 1.0 & 1.0 & 1.0 & 222.49 \\
\hline 0.1 & 0.1 & 0.1 & 0.5 & 0.1 & 0.3 & -0.9 & 0.5 & 0.3 & 1.0 & 1.0 & 1.0 & 248.65 \\
\hline 0.1 & 0.1 & 0.1 & 0.5 & 0.1 & 0.3 & -0.9 & 0.5 & 0.5 & 1.0 & 1.0 & 1.0 & 281.79 \\
\hline 0.1 & 0.1 & 0.1 & 0.5 & 0.1 & 0.3 & -0.9 & 0.5 & 0.7 & 1.0 & 1.0 & 1.0 & 325.11 \\
\hline 0.1 & 0.1 & 0.1 & 0.5 & 0.1 & 0.3 & -0.9 & 0.7 & 0.1 & 1.0 & 1.0 & 1.0 & 469.47 \\
\hline 0.1 & 0.1 & 0.1 & 0.5 & 0.1 & 0.3 & -0.7 & 0.5 & 0.3 & 1.0 & 1.0 & 1.0 & 207.91 \\
\hline 0.1 & 0.1 & 0.1 & 0.5 & 0.1 & 0.3 & -0.7 & 0.5 & 0.5 & 1.0 & 1.0 & 1.0 & 230.58 \\
\hline 0.1 & 0.1 & 0.1 & 0.5 & 0.1 & 0.3 & -0.7 & 0.5 & 0.7 & 1.0 & 1.0 & 1.0 & 258.80 \\
\hline 0.1 & 0.1 & 0.1 & 0.5 & 0.1 & 0.3 & -0.7 & 0.7 & 0.1 & 1.0 & 1.0 & 1.0 & 342.67 \\
\hline
\end{tabular}


ODUMADE \& SINGH

Appendix

Table 3.1 (continued): Relative efficiency of the proposed ratio estimator with respect to the BBB model for different choice of parameters with $P=P_{1}=P_{2}=0.7$

\begin{tabular}{|c|c|c|c|c|c|c|c|c|c|c|c|c|}
\hline$C_{\gamma}$ & $C_{\gamma_{1}}$ & $C_{\gamma_{2}}$ & $C_{y}$ & $C x_{1}$ & $C x_{2}$ & $\rho_{y x_{1}}$ & $\rho_{y x_{2}}$ & $\rho_{x_{1} x_{2}}$ & $\theta$ & $\theta_{1}$ & $\theta_{2}$ & $\mathrm{RE}$ \\
\hline 0.1 & 0.1 & 0.1 & 0.5 & 0.1 & 0.3 & -0.7 & 0.7 & 0.3 & 1.0 & 1.0 & 1.0 & 408.94 \\
\hline 0.1 & 0.1 & 0.1 & 0.5 & 0.1 & 0.3 & -0.5 & 0.5 & 0.7 & 1.0 & 1.0 & 1.0 & 214.95 \\
\hline 0.1 & 0.1 & 0.1 & 0.5 & 0.1 & 0.3 & -0.5 & 0.7 & 0.1 & 1.0 & 1.0 & 1.0 & 269.80 \\
\hline 0.1 & 0.1 & 0.1 & 0.5 & 0.1 & 0.3 & -0.5 & 0.7 & 0.3 & 1.0 & 1.0 & 1.0 & 309.26 \\
\hline 0.1 & 0.1 & 0.1 & 0.5 & 0.1 & 0.3 & -0.5 & 0.7 & 0.5 & 1.0 & 1.0 & 1.0 & 362.24 \\
\hline 0.1 & 0.1 & 0.1 & 0.5 & 0.1 & 0.3 & -0.5 & 0.7 & 0.7 & 1.0 & 1.0 & 1.0 & 437.12 \\
\hline 0.1 & 0.1 & 0.1 & 0.5 & 0.1 & 0.3 & -0.3 & 0.7 & 0.1 & 1.0 & 1.0 & 1.0 & 222.49 \\
\hline 0.1 & 0.1 & 0.1 & 0.5 & 0.1 & 0.3 & -0.3 & 0.7 & 0.3 & 1.0 & 1.0 & 1.0 & 248.65 \\
\hline 0.1 & 0.1 & 0.1 & 0.5 & 0.1 & 0.3 & -0.3 & 0.7 & 0.5 & 1.0 & 1.0 & 1.0 & 281.79 \\
\hline 0.1 & 0.1 & 0.1 & 0.5 & 0.1 & 0.3 & -0.3 & 0.7 & 0.7 & 1.0 & 1.0 & 1.0 & 325.11 \\
\hline 0.1 & 0.1 & 0.1 & 0.5 & 0.1 & 0.3 & -0.1 & 0.7 & 0.3 & 1.0 & 1.0 & 1.0 & 207.91 \\
\hline 0.1 & 0.1 & 0.1 & 0.5 & 0.1 & 0.3 & -0.1 & 0.7 & 0.5 & 1.0 & 1.0 & 1.0 & 230.58 \\
\hline 0.1 & 0.1 & 0.1 & 0.5 & 0.1 & 0.3 & -0.1 & 0.7 & 0.7 & 1.0 & 1.0 & 1.0 & 258.80 \\
\hline 0.1 & 0.1 & 0.1 & 0.5 & 0.1 & 0.3 & 0.1 & 0.7 & 0.7 & 1.0 & 1.0 & 1.0 & 214.95 \\
\hline 0.1 & 0.1 & 0.1 & 0.5 & 0.1 & 0.5 & -0.9 & 0.5 & 0.7 & 1.0 & 1.0 & 1.0 & 229.58 \\
\hline 0.1 & 0.1 & 0.1 & 0.5 & 0.1 & 0.5 & -0.9 & 0.7 & 0.1 & 1.0 & 1.0 & 1.0 & 359.78 \\
\hline 0.1 & 0.1 & 0.1 & 0.5 & 0.1 & 0.5 & -0.7 & 0.7 & 0.1 & 1.0 & 1.0 & 1.0 & 280.29 \\
\hline 0.1 & 0.1 & 0.1 & 0.5 & 0.1 & 0.5 & -0.7 & 0.7 & 0.3 & 1.0 & 1.0 & 1.0 & 359.78 \\
\hline 0.1 & 0.1 & 0.1 & 0.5 & 0.1 & 0.5 & -0.5 & 0.7 & 0.1 & 1.0 & 1.0 & 1.0 & 229.58 \\
\hline 0.1 & 0.1 & 0.1 & 0.5 & 0.1 & 0.5 & -0.5 & 0.7 & 0.3 & 1.0 & 1.0 & 1.0 & 280.29 \\
\hline 0.1 & 0.1 & 0.1 & 0.5 & 0.1 & 0.5 & -0.5 & 0.7 & 0.5 & 1.0 & 1.0 & 1.0 & 359.78 \\
\hline 0.1 & 0.1 & 0.1 & 0.5 & 0.1 & 0.5 & -0.3 & 0.7 & 0.3 & 1.0 & 1.0 & 1.0 & 229.58 \\
\hline 0.1 & 0.1 & 0.1 & 0.5 & 0.1 & 0.5 & -0.3 & 0.7 & 0.5 & 1.0 & 1.0 & 1.0 & 280.29 \\
\hline 0.1 & 0.1 & 0.1 & 0.5 & 0.1 & 0.5 & -0.3 & 0.7 & 0.7 & 1.0 & 1.0 & 1.0 & 359.78 \\
\hline 0.1 & 0.1 & 0.1 & 0.5 & 0.1 & 0.5 & -0.1 & 0.7 & 0.5 & 1.0 & 1.0 & 1.0 & 229.58 \\
\hline 0.1 & 0.1 & 0.1 & 0.5 & 0.1 & 0.5 & -0.1 & 0.7 & 0.7 & 1.0 & 1.0 & 1.0 & 280.29 \\
\hline 0.1 & 0.1 & 0.1 & 0.5 & 0.1 & 0.5 & 0.1 & 0.7 & 0.7 & 1.0 & 1.0 & 1.0 & 229.58 \\
\hline 0.1 & 0.1 & 0.1 & 0.5 & 0.3 & 0.1 & -0.9 & 0.1 & 0.1 & 1.0 & 1.0 & 1.0 & 342.67 \\
\hline 0.1 & 0.1 & 0.1 & 0.5 & 0.3 & 0.1 & -0.9 & 0.1 & 0.3 & 1.0 & 1.0 & 1.0 & 408.94 \\
\hline 0.1 & 0.1 & 0.1 & 0.5 & 0.3 & 0.1 & -0.9 & 0.3 & 0.1 & 1.0 & 1.0 & 1.0 & 469.47 \\
\hline 0.1 & 0.1 & 0.1 & 0.5 & 0.3 & 0.1 & -0.7 & 0.1 & 0.3 & 1.0 & 1.0 & 1.0 & 207.91 \\
\hline 0.1 & 0.1 & 0.1 & 0.5 & 0.3 & 0.1 & -0.7 & 0.1 & 0.5 & 1.0 & 1.0 & 1.0 & 230.58 \\
\hline 0.1 & 0.1 & 0.1 & 0.5 & 0.3 & 0.1 & -0.7 & 0.1 & 0.7 & 1.0 & 1.0 & 1.0 & 258.80 \\
\hline 0.1 & 0.1 & 0.1 & 0.5 & 0.3 & 0.1 & -0.7 & 0.3 & 0.1 & 1.0 & 1.0 & 1.0 & 222.49 \\
\hline 0.1 & 0.1 & 0.1 & 0.5 & 0.3 & 0.1 & -0.7 & 0.3 & 0.3 & 1.0 & 1.0 & 1.0 & 248.65 \\
\hline 0.1 & 0.1 & 0.1 & 0.5 & 0.3 & 0.1 & -0.7 & 0.3 & 0.5 & 1.0 & 1.0 & 1.0 & 281.79 \\
\hline 0.1 & 0.1 & 0.1 & 0.5 & 0.3 & 0.1 & -0.7 & 0.3 & 0.7 & 1.0 & 1.0 & 1.0 & 325.11 \\
\hline 0.1 & 0.1 & 0.1 & 0.5 & 0.3 & 0.1 & -0.7 & 0.5 & 0.1 & 1.0 & 1.0 & 1.0 & 269.80 \\
\hline 0.1 & 0.1 & 0.1 & 0.5 & 0.3 & 0.1 & -0.7 & 0.5 & 0.3 & 1.0 & 1.0 & 1.0 & 309.26 \\
\hline 0.1 & 0.1 & 0.1 & 0.5 & 0.3 & 0.1 & -0.7 & 0.5 & 0.5 & 1.0 & 1.0 & 1.0 & 362.24 \\
\hline
\end{tabular}


IMPROVING THE BAR-LEV, BOBOVITCH \& BOUKAI RANDOM RESPONSE MODELS

Appendix

Table 3.1 (continued): Relative efficiency of the proposed ratio estimator with respect to the BBB model for different choice of parameters with $P=P_{1}=P_{2}=0.7$

\begin{tabular}{|c|c|c|c|c|c|c|c|c|c|c|c|c|}
\hline$C_{\gamma}$ & $C_{\gamma_{1}}$ & $C_{\gamma_{2}}$ & $C_{y}$ & $C x_{1}$ & $C x_{2}$ & $\rho_{y x_{1}}$ & $\rho_{y x_{2}}$ & $\rho_{x_{1} x_{2}}$ & $\theta$ & $\theta_{1}$ & $\theta_{2}$ & $\mathrm{RE}$ \\
\hline 0.1 & 0.1 & 0.1 & 0.5 & 0.3 & 0.1 & -0.7 & 0.5 & 0.7 & 1.0 & 1.0 & 1.0 & 437.12 \\
\hline 0.1 & 0.1 & 0.1 & 0.5 & 0.3 & 0.1 & -0.7 & 0.7 & 0.1 & 1.0 & 1.0 & 1.0 & 342.67 \\
\hline 0.1 & 0.1 & 0.1 & 0.5 & 0.3 & 0.1 & -0.7 & 0.7 & 0.3 & 1.0 & 1.0 & 1.0 & 408.94 \\
\hline 0.1 & 0.1 & 0.1 & 0.5 & 0.3 & 0.1 & -0.5 & 0.5 & 0.7 & 1.0 & 1.0 & 1.0 & 214.95 \\
\hline 0.1 & 0.1 & 0.1 & 0.5 & 0.3 & 0.1 & -0.5 & 0.7 & 0.3 & 1.0 & 1.0 & 1.0 & 207.91 \\
\hline 0.1 & 0.1 & 0.1 & 0.5 & 0.3 & 0.1 & -0.5 & 0.7 & 0.5 & 1.0 & 1.0 & 1.0 & 230.58 \\
\hline 0.1 & 0.1 & 0.1 & 0.5 & 0.3 & 0.1 & -0.5 & 0.7 & 0.7 & 1.0 & 1.0 & 1.0 & 258.80 \\
\hline 0.1 & 0.1 & 0.1 & 0.5 & 0.3 & 0.3 & -0.9 & 0.1 & 0.1 & 1.0 & 1.0 & 1.0 & 207.50 \\
\hline 0.1 & 0.1 & 0.1 & 0.5 & 0.3 & 0.3 & -0.9 & 0.1 & 0.3 & 1.0 & 1.0 & 1.0 & 294.07 \\
\hline 0.1 & 0.1 & 0.1 & 0.5 & 0.3 & 0.3 & -0.9 & 0.3 & 0.1 & 1.0 & 1.0 & 1.0 & 407.37 \\
\hline 0.1 & 0.1 & 0.1 & 0.5 & 0.3 & 0.3 & -0.7 & 0.1 & 0.5 & 1.0 & 1.0 & 1.0 & 230.08 \\
\hline 0.1 & 0.1 & 0.1 & 0.5 & 0.3 & 0.3 & -0.7 & 0.1 & 0.7 & 1.0 & 1.0 & 1.0 & 341.57 \\
\hline 0.1 & 0.1 & 0.1 & 0.5 & 0.3 & 0.3 & -0.7 & 0.3 & 0.1 & 1.0 & 1.0 & 1.0 & 207.50 \\
\hline 0.1 & 0.1 & 0.1 & 0.5 & 0.3 & 0.3 & -0.7 & 0.3 & 0.3 & 1.0 & 1.0 & 1.0 & 294.07 \\
\hline 0.1 & 0.1 & 0.1 & 0.5 & 0.3 & 0.3 & -0.7 & 0.5 & 0.1 & 1.0 & 1.0 & 1.0 & 407.37 \\
\hline 0.1 & 0.1 & 0.1 & 0.5 & 0.3 & 0.3 & -0.5 & 0.3 & 0.5 & 1.0 & 1.0 & 1.0 & 230.08 \\
\hline 0.1 & 0.1 & 0.1 & 0.5 & 0.3 & 0.3 & -0.5 & 0.3 & 0.7 & 1.0 & 1.0 & 1.0 & 341.57 \\
\hline 0.1 & 0.1 & 0.1 & 0.5 & 0.3 & 0.3 & -0.5 & 0.5 & 0.1 & 1.0 & 1.0 & 1.0 & 207.50 \\
\hline 0.1 & 0.1 & 0.1 & 0.5 & 0.3 & 0.3 & -0.5 & 0.5 & 0.3 & 1.0 & 1.0 & 1.0 & 294.07 \\
\hline 0.1 & 0.1 & 0.1 & 0.5 & 0.3 & 0.3 & -0.5 & 0.7 & 0.1 & 1.0 & 1.0 & 1.0 & 407.37 \\
\hline 0.1 & 0.1 & 0.1 & 0.5 & 0.3 & 0.3 & -0.3 & 0.5 & 0.5 & 1.0 & 1.0 & 1.0 & 230.08 \\
\hline 0.1 & 0.1 & 0.1 & 0.5 & 0.3 & 0.3 & -0.3 & 0.5 & 0.7 & 1.0 & 1.0 & 1.0 & 341.57 \\
\hline 0.1 & 0.1 & 0.1 & 0.5 & 0.3 & 0.3 & -0.3 & 0.7 & 0.1 & 1.0 & 1.0 & 1.0 & 207.50 \\
\hline 0.1 & 0.1 & 0.1 & 0.5 & 0.3 & 0.3 & -0.3 & 0.7 & 0.3 & 1.0 & 1.0 & 1.0 & 294.07 \\
\hline 0.1 & 0.1 & 0.1 & 0.5 & 0.3 & 0.3 & -0.1 & 0.7 & 0.5 & 1.0 & 1.0 & 1.0 & 230.08 \\
\hline 0.1 & 0.1 & 0.1 & 0.5 & 0.3 & 0.3 & -0.1 & 0.7 & 0.7 & 1.0 & 1.0 & 1.0 & 341.57 \\
\hline 0.1 & 0.1 & 0.1 & 0.5 & 0.3 & 0.5 & -0.9 & 0.1 & 0.7 & 1.0 & 1.0 & 1.0 & 358.56 \\
\hline 0.1 & 0.1 & 0.1 & 0.5 & 0.3 & 0.5 & -0.9 & 0.3 & 0.3 & 1.0 & 1.0 & 1.0 & 279.55 \\
\hline 0.1 & 0.1 & 0.1 & 0.5 & 0.3 & 0.5 & -0.9 & 0.5 & 0.1 & 1.0 & 1.0 & 1.0 & 499.80 \\
\hline 0.1 & 0.1 & 0.1 & 0.5 & 0.3 & 0.5 & -0.7 & 0.3 & 0.5 & 1.0 & 1.0 & 1.0 & 279.55 \\
\hline 0.1 & 0.1 & 0.1 & 0.5 & 0.3 & 0.5 & -0.7 & 0.5 & 0.1 & 1.0 & 1.0 & 1.0 & 229.08 \\
\hline 0.1 & 0.1 & 0.1 & 0.5 & 0.3 & 0.5 & -0.7 & 0.5 & 0.3 & 1.0 & 1.0 & 1.0 & 499.80 \\
\hline 0.1 & 0.1 & 0.1 & 0.5 & 0.3 & 0.5 & -0.5 & 0.3 & 0.7 & 1.0 & 1.0 & 1.0 & 279.55 \\
\hline 0.1 & 0.1 & 0.1 & 0.5 & 0.3 & 0.5 & -0.5 & 0.5 & 0.3 & 1.0 & 1.0 & 1.0 & 229.08 \\
\hline 0.1 & 0.1 & 0.1 & 0.5 & 0.3 & 0.5 & -0.5 & 0.5 & 0.5 & 1.0 & 1.0 & 1.0 & 499.80 \\
\hline 0.1 & 0.1 & 0.1 & 0.5 & 0.3 & 0.5 & -0.5 & 0.7 & 0.1 & 1.0 & 1.0 & 1.0 & 358.56 \\
\hline 0.1 & 0.1 & 0.1 & 0.5 & 0.3 & 0.5 & -0.3 & 0.5 & 0.5 & 1.0 & 1.0 & 1.0 & 229.08 \\
\hline 0.1 & 0.1 & 0.1 & 0.5 & 0.3 & 0.5 & -0.3 & 0.5 & 0.7 & 1.0 & 1.0 & 1.0 & 499.80 \\
\hline 0.1 & 0.1 & 0.1 & 0.5 & 0.3 & 0.5 & -0.3 & 0.7 & 0.3 & 1.0 & 1.0 & 1.0 & 358.56 \\
\hline 0.1 & 0.1 & 0.1 & 0.5 & 0.3 & 0.5 & -0.1 & 0.5 & 0.7 & 1.0 & 1.0 & 1.0 & 229.08 \\
\hline
\end{tabular}


ODUMADE \& SINGH

Appendix

Table 3.1 (continued): Relative efficiency of the proposed ratio estimator with respect to the BBB model for different choice of parameters with $P=P_{1}=P_{2}=0.7$

\begin{tabular}{|c|c|c|c|c|c|c|c|c|c|c|c|c|}
\hline$C_{\gamma}$ & $C_{\gamma_{1}}$ & $C_{\gamma_{2}}$ & $C_{y}$ & $C x_{1}$ & $C x_{2}$ & $\rho_{y x_{1}}$ & $\rho_{y x_{2}}$ & $\rho_{x_{1} x_{2}}$ & $\theta$ & $\theta_{1}$ & $\theta_{2}$ & $\mathrm{RE}$ \\
\hline 0.1 & 0.1 & 0.1 & 0.5 & 0.3 & 0.5 & -0.1 & 0.7 & 0.5 & 1.0 & 1.0 & 1.0 & 358.56 \\
\hline 0.1 & 0.1 & 0.1 & 0.5 & 0.3 & 0.5 & 0.1 & 0.7 & 0.7 & 1.0 & 1.0 & 1.0 & 358.56 \\
\hline 0.1 & 0.1 & 0.1 & 0.5 & 0.5 & 0.1 & -0.7 & 0.1 & 0.5 & 1.0 & 1.0 & 1.0 & 229.58 \\
\hline 0.1 & 0.1 & 0.1 & 0.5 & 0.5 & 0.1 & -0.7 & 0.1 & 0.7 & 1.0 & 1.0 & 1.0 & 280.29 \\
\hline 0.1 & 0.1 & 0.1 & 0.5 & 0.5 & 0.1 & -0.7 & 0.3 & 0.3 & 1.0 & 1.0 & 1.0 & 229.58 \\
\hline 0.1 & 0.1 & 0.1 & 0.5 & 0.5 & 0.1 & -0.7 & 0.3 & 0.5 & 1.0 & 1.0 & 1.0 & 280.29 \\
\hline 0.1 & 0.1 & 0.1 & 0.5 & 0.5 & 0.1 & -0.7 & 0.3 & 0.7 & 1.0 & 1.0 & 1.0 & 359.78 \\
\hline 0.1 & 0.1 & 0.1 & 0.5 & 0.5 & 0.1 & -0.7 & 0.5 & 0.1 & 1.0 & 1.0 & 1.0 & 229.58 \\
\hline 0.1 & 0.1 & 0.1 & 0.5 & 0.5 & 0.1 & -0.7 & 0.5 & 0.3 & 1.0 & 1.0 & 1.0 & 280.29 \\
\hline 0.1 & 0.1 & 0.1 & 0.5 & 0.5 & 0.1 & -0.7 & 0.5 & 0.5 & 1.0 & 1.0 & 1.0 & 359.78 \\
\hline 0.1 & 0.1 & 0.1 & 0.5 & 0.5 & 0.1 & -0.7 & 0.7 & 0.1 & 1.0 & 1.0 & 1.0 & 280.29 \\
\hline 0.1 & 0.1 & 0.1 & 0.5 & 0.5 & 0.1 & -0.7 & 0.7 & 0.3 & 1.0 & 1.0 & 1.0 & 359.78 \\
\hline 0.1 & 0.1 & 0.1 & 0.5 & 0.5 & 0.3 & -0.9 & 0.1 & 0.1 & 1.0 & 1.0 & 1.0 & 279.55 \\
\hline 0.1 & 0.1 & 0.1 & 0.5 & 0.5 & 0.3 & -0.7 & 0.1 & 0.5 & 1.0 & 1.0 & 1.0 & 358.56 \\
\hline 0.1 & 0.1 & 0.1 & 0.5 & 0.5 & 0.3 & -0.7 & 0.3 & 0.3 & 1.0 & 1.0 & 1.0 & 358.56 \\
\hline 0.1 & 0.1 & 0.1 & 0.5 & 0.5 & 0.3 & -0.7 & 0.5 & 0.1 & 1.0 & 1.0 & 1.0 & 358.56 \\
\hline 0.1 & 0.1 & 0.1 & 0.5 & 0.5 & 0.3 & -0.5 & 0.1 & 0.7 & 1.0 & 1.0 & 1.0 & 229.08 \\
\hline 0.1 & 0.1 & 0.1 & 0.5 & 0.5 & 0.3 & -0.5 & 0.3 & 0.5 & 1.0 & 1.0 & 1.0 & 229.08 \\
\hline 0.1 & 0.1 & 0.1 & 0.5 & 0.5 & 0.3 & -0.5 & 0.3 & 0.7 & 1.0 & 1.0 & 1.0 & 499.80 \\
\hline 0.1 & 0.1 & 0.1 & 0.5 & 0.5 & 0.3 & -0.5 & 0.5 & 0.3 & 1.0 & 1.0 & 1.0 & 229.08 \\
\hline 0.1 & 0.1 & 0.1 & 0.5 & 0.5 & 0.3 & -0.5 & 0.5 & 0.5 & 1.0 & 1.0 & 1.0 & 499.80 \\
\hline 0.1 & 0.1 & 0.1 & 0.5 & 0.5 & 0.3 & -0.5 & 0.7 & 0.1 & 1.0 & 1.0 & 1.0 & 229.08 \\
\hline 0.1 & 0.1 & 0.1 & 0.5 & 0.5 & 0.3 & -0.5 & 0.7 & 0.3 & 1.0 & 1.0 & 1.0 & 499.80 \\
\hline 0.1 & 0.1 & 0.1 & 0.5 & 0.5 & 0.3 & -0.3 & 0.5 & 0.7 & 1.0 & 1.0 & 1.0 & 279.55 \\
\hline 0.1 & 0.1 & 0.1 & 0.5 & 0.5 & 0.3 & -0.3 & 0.7 & 0.5 & 1.0 & 1.0 & 1.0 & 279.55 \\
\hline 0.1 & 0.1 & 0.1 & 0.5 & 0.5 & 0.5 & -0.9 & 0.1 & 0.3 & 1.0 & 1.0 & 1.0 & 228.09 \\
\hline 0.1 & 0.1 & 0.1 & 0.5 & 0.5 & 0.5 & -0.9 & 0.3 & 0.1 & 1.0 & 1.0 & 1.0 & 228.09 \\
\hline 0.1 & 0.1 & 0.1 & 0.5 & 0.5 & 0.5 & -0.7 & 0.1 & 0.5 & 1.0 & 1.0 & 1.0 & 228.09 \\
\hline 0.1 & 0.1 & 0.1 & 0.5 & 0.5 & 0.5 & -0.7 & 0.3 & 0.3 & 1.0 & 1.0 & 1.0 & 228.09 \\
\hline 0.1 & 0.1 & 0.1 & 0.5 & 0.5 & 0.5 & -0.7 & 0.5 & 0.1 & 1.0 & 1.0 & 1.0 & 228.09 \\
\hline 0.1 & 0.1 & 0.1 & 0.5 & 0.5 & 0.5 & -0.5 & 0.1 & 0.7 & 1.0 & 1.0 & 1.0 & 228.09 \\
\hline 0.1 & 0.1 & 0.1 & 0.5 & 0.5 & 0.5 & -0.5 & 0.3 & 0.5 & 1.0 & 1.0 & 1.0 & 228.09 \\
\hline 0.1 & 0.1 & 0.1 & 0.5 & 0.5 & 0.5 & -0.5 & 0.5 & 0.3 & 1.0 & 1.0 & 1.0 & 228.09 \\
\hline 0.1 & 0.1 & 0.1 & 0.5 & 0.5 & 0.5 & -0.5 & 0.7 & 0.1 & 1.0 & 1.0 & 1.0 & 228.09 \\
\hline 0.1 & 0.1 & 0.1 & 0.5 & 0.5 & 0.5 & -0.3 & 0.3 & 0.7 & 1.0 & 1.0 & 1.0 & 228.09 \\
\hline 0.1 & 0.1 & 0.1 & 0.5 & 0.5 & 0.5 & -0.3 & 0.5 & 0.5 & 1.0 & 1.0 & 1.0 & 228.09 \\
\hline 0.1 & 0.1 & 0.1 & 0.5 & 0.5 & 0.5 & -0.3 & 0.7 & 0.3 & 1.0 & 1.0 & 1.0 & 228.09 \\
\hline 0.1 & 0.1 & 0.1 & 0.5 & 0.5 & 0.5 & -0.1 & 0.5 & 0.7 & 1.0 & 1.0 & 1.0 & 228.09 \\
\hline 0.1 & 0.1 & 0.1 & 0.5 & 0.5 & 0.5 & -0.1 & 0.7 & 0.5 & 1.0 & 1.0 & 1.0 & 228.09 \\
\hline 0.1 & 0.1 & 0.1 & 0.5 & 0.5 & 0.5 & 0.1 & 0.7 & 0.7 & 1.0 & 1.0 & 1.0 & 228.09 \\
\hline
\end{tabular}


IMPROVING THE BAR-LEV, BOBOVITCH \& BOUKAI RANDOM RESPONSE MODELS

Appendix

Table 3.1 (continued): Relative efficiency of the proposed ratio estimator with respect to the BBB model for different choice of parameters with $P=P_{1}=P_{2}=0.7$

\begin{tabular}{|c|c|c|c|c|c|c|c|c|c|c|c|c|}
\hline$C_{\gamma}$ & $C_{\gamma_{1}}$ & $C_{\gamma_{2}}$ & $C_{y}$ & $C x_{1}$ & $C x_{2}$ & $\rho_{y x_{1}}$ & $\rho_{y x_{2}}$ & $\rho_{x_{1} x_{2}}$ & $\theta$ & $\theta_{1}$ & $\theta_{2}$ & $\mathrm{RE}$ \\
\hline 0.3 & 0.3 & 0.3 & 0.3 & 0.1 & 0.3 & -0.9 & 0.7 & 0.7 & 1.0 & 1.0 & 1.0 & 220.64 \\
\hline 0.3 & 0.3 & 0.3 & 0.3 & 0.3 & 0.1 & -0.9 & 0.3 & 0.7 & 1.0 & 1.0 & 1.0 & 220.64 \\
\hline 0.3 & 0.3 & 0.3 & 0.3 & 0.3 & 0.1 & -0.9 & 0.5 & 0.5 & 1.0 & 1.0 & 1.0 & 220.64 \\
\hline 0.3 & 0.3 & 0.3 & 0.3 & 0.3 & 0.1 & -0.9 & 0.5 & 0.7 & 1.0 & 1.0 & 1.0 & 283.48 \\
\hline 0.3 & 0.3 & 0.3 & 0.3 & 0.3 & 0.1 & -0.9 & 0.7 & 0.3 & 1.0 & 1.0 & 1.0 & 220.64 \\
\hline 0.3 & 0.3 & 0.3 & 0.3 & 0.3 & 0.1 & -0.9 & 0.7 & 0.5 & 1.0 & 1.0 & 1.0 & 283.48 \\
\hline 0.3 & 0.3 & 0.3 & 0.3 & 0.3 & 0.1 & -0.9 & 0.7 & 0.7 & 1.0 & 1.0 & 1.0 & 396.38 \\
\hline 0.3 & 0.3 & 0.3 & 0.3 & 0.3 & 0.3 & -0.9 & 0.1 & 0.7 & 1.0 & 1.0 & 1.0 & 228.40 \\
\hline 0.3 & 0.3 & 0.3 & 0.3 & 0.3 & 0.3 & -0.9 & 0.3 & 0.5 & 1.0 & 1.0 & 1.0 & 228.40 \\
\hline 0.3 & 0.3 & 0.3 & 0.3 & 0.3 & 0.3 & -0.9 & 0.5 & 0.3 & 1.0 & 1.0 & 1.0 & 228.40 \\
\hline 0.3 & 0.3 & 0.3 & 0.3 & 0.3 & 0.3 & -0.9 & 0.7 & 0.1 & 1.0 & 1.0 & 1.0 & 228.40 \\
\hline 0.3 & 0.3 & 0.3 & 0.3 & 0.3 & 0.3 & -0.7 & 0.3 & 0.7 & 1.0 & 1.0 & 1.0 & 228.40 \\
\hline 0.3 & 0.3 & 0.3 & 0.3 & 0.3 & 0.3 & -0.7 & 0.5 & 0.5 & 1.0 & 1.0 & 1.0 & 228.40 \\
\hline 0.3 & 0.3 & 0.3 & 0.3 & 0.3 & 0.3 & -0.7 & 0.7 & 0.3 & 1.0 & 1.0 & 1.0 & 228.40 \\
\hline 0.3 & 0.3 & 0.3 & 0.3 & 0.3 & 0.3 & -0.5 & 0.5 & 0.7 & 1.0 & 1.0 & 1.0 & 228.40 \\
\hline 0.3 & 0.3 & 0.3 & 0.3 & 0.3 & 0.3 & -0.5 & 0.7 & 0.5 & 1.0 & 1.0 & 1.0 & 228.40 \\
\hline 0.3 & 0.3 & 0.3 & 0.3 & 0.3 & 0.3 & -0.3 & 0.7 & 0.7 & 1.0 & 1.0 & 1.0 & 228.40 \\
\hline 0.3 & 0.3 & 0.3 & 0.3 & 0.3 & 0.5 & -0.7 & 0.5 & 0.7 & 1.0 & 1.0 & 1.0 & 326.22 \\
\hline 0.3 & 0.3 & 0.3 & 0.3 & 0.3 & 0.5 & -0.7 & 0.7 & 0.5 & 1.0 & 1.0 & 1.0 & 326.22 \\
\hline 0.3 & 0.3 & 0.3 & 0.3 & 0.3 & 0.5 & -0.3 & 0.7 & 0.7 & 1.0 & 1.0 & 1.0 & 245.69 \\
\hline 0.3 & 0.3 & 0.3 & 0.3 & 0.5 & 0.1 & -0.9 & 0.7 & 0.7 & 1.0 & 1.0 & 1.0 & 204.33 \\
\hline 0.3 & 0.3 & 0.3 & 0.3 & 0.5 & 0.3 & -0.9 & 0.1 & 0.7 & 1.0 & 1.0 & 1.0 & 485.29 \\
\hline 0.3 & 0.3 & 0.3 & 0.3 & 0.5 & 0.3 & -0.9 & 0.3 & 0.5 & 1.0 & 1.0 & 1.0 & 245.69 \\
\hline 0.3 & 0.3 & 0.3 & 0.3 & 0.5 & 0.3 & -0.9 & 0.7 & 0.3 & 1.0 & 1.0 & 1.0 & 326.22 \\
\hline 0.3 & 0.3 & 0.3 & 0.3 & 0.5 & 0.3 & -0.7 & 0.3 & 0.7 & 1.0 & 1.0 & 1.0 & 245.69 \\
\hline 0.3 & 0.3 & 0.3 & 0.3 & 0.5 & 0.3 & -0.7 & 0.7 & 0.5 & 1.0 & 1.0 & 1.0 & 326.22 \\
\hline 0.3 & 0.3 & 0.3 & 0.3 & 0.5 & 0.3 & -0.5 & 0.7 & 0.7 & 1.0 & 1.0 & 1.0 & 326.22 \\
\hline 0.3 & 0.3 & 0.3 & 0.3 & 0.5 & 0.5 & -0.9 & 0.1 & 0.7 & 1.0 & 1.0 & 1.0 & 323.40 \\
\hline 0.3 & 0.3 & 0.3 & 0.3 & 0.5 & 0.5 & -0.9 & 0.7 & 0.3 & 1.0 & 1.0 & 1.0 & 209.78 \\
\hline 0.3 & 0.3 & 0.3 & 0.3 & 0.5 & 0.5 & -0.7 & 0.3 & 0.7 & 1.0 & 1.0 & 1.0 & 323.40 \\
\hline 0.3 & 0.3 & 0.3 & 0.3 & 0.5 & 0.5 & -0.5 & 0.5 & 0.7 & 1.0 & 1.0 & 1.0 & 323.40 \\
\hline 0.3 & 0.3 & 0.3 & 0.3 & 0.5 & 0.5 & -0.3 & 0.7 & 0.7 & 1.0 & 1.0 & 1.0 & 323.40 \\
\hline 0.3 & 0.3 & 0.3 & 0.5 & 0.1 & 0.3 & -0.9 & 0.7 & 0.1 & 1.0 & 1.0 & 1.0 & 211.04 \\
\hline 0.3 & 0.3 & 0.3 & 0.5 & 0.1 & 0.3 & -0.9 & 0.7 & 0.3 & 1.0 & 1.0 & 1.0 & 231.73 \\
\hline 0.3 & 0.3 & 0.3 & 0.5 & 0.1 & 0.3 & -0.9 & 0.7 & 0.5 & 1.0 & 1.0 & 1.0 & 256.90 \\
\hline 0.3 & 0.3 & 0.3 & 0.5 & 0.1 & 0.3 & -0.9 & 0.7 & 0.7 & 1.0 & 1.0 & 1.0 & 288.22 \\
\hline 0.3 & 0.3 & 0.3 & 0.5 & 0.1 & 0.3 & -0.7 & 0.7 & 0.5 & 1.0 & 1.0 & 1.0 & 217.52 \\
\hline 0.3 & 0.3 & 0.3 & 0.5 & 0.1 & 0.3 & -0.7 & 0.7 & 0.7 & 1.0 & 1.0 & 1.0 & 239.55 \\
\hline 0.3 & 0.3 & 0.3 & 0.5 & 0.1 & 0.3 & -0.5 & 0.7 & 0.7 & 1.0 & 1.0 & 1.0 & 204.95 \\
\hline 0.3 & 0.3 & 0.3 & 0.5 & 0.1 & 0.5 & -0.9 & 0.7 & 0.3 & 1.0 & 1.0 & 1.0 & 210.54 \\
\hline 0.3 & 0.3 & 0.3 & 0.5 & 0.1 & 0.5 & -0.9 & 0.7 & 0.5 & 1.0 & 1.0 & 1.0 & 247.23 \\
\hline
\end{tabular}




\section{ODUMADE \& SINGH}

\section{Appendix}

Table 3.1 (continued): Relative efficiency of the proposed ratio estimator with respect to the BBB model for different choice of parameters with $P=P_{1}=P_{2}=0.7$

\begin{tabular}{|c|c|c|c|c|c|c|c|c|c|c|c|c|}
\hline$C_{\gamma}$ & $C_{\gamma_{1}}$ & $C_{\gamma_{2}}$ & $C_{y}$ & $C x_{1}$ & $C x_{2}$ & $\rho_{y x_{1}}$ & $\rho_{y x_{2}}$ & $\rho_{x_{1} x_{2}}$ & $\theta$ & $\theta_{1}$ & $\theta_{2}$ & $\mathrm{RE}$ \\
\hline 0.3 & 0.3 & 0.3 & 0.5 & 0.1 & 0.5 & -0.9 & 0.7 & 0.7 & 1.0 & 1.0 & 1.0 & 299.41 \\
\hline 0.3 & 0.3 & 0.3 & 0.5 & 0.1 & 0.5 & -0.7 & 0.7 & 0.5 & 1.0 & 1.0 & 1.0 & 210.54 \\
\hline 0.3 & 0.3 & 0.3 & 0.5 & 0.1 & 0.5 & -0.7 & 0.7 & 0.7 & 1.0 & 1.0 & 1.0 & 247.23 \\
\hline 0.3 & 0.3 & 0.3 & 0.5 & 0.1 & 0.5 & -0.5 & 0.7 & 0.7 & 1.0 & 1.0 & 1.0 & 210.54 \\
\hline 0.3 & 0.3 & 0.3 & 0.5 & 0.3 & 0.1 & -0.9 & 0.1 & 0.5 & 1.0 & 1.0 & 1.0 & 217.52 \\
\hline 0.3 & 0.3 & 0.3 & 0.5 & 0.3 & 0.1 & -0.9 & 0.1 & 0.7 & 1.0 & 1.0 & 1.0 & 239.55 \\
\hline 0.3 & 0.3 & 0.3 & 0.5 & 0.3 & 0.1 & -0.9 & 0.3 & 0.1 & 1.0 & 1.0 & 1.0 & 211.04 \\
\hline 0.3 & 0.3 & 0.3 & 0.5 & 0.3 & 0.1 & -0.9 & 0.3 & 0.3 & 1.0 & 1.0 & 1.0 & 231.73 \\
\hline 0.3 & 0.3 & 0.3 & 0.5 & 0.3 & 0.1 & -0.9 & 0.3 & 0.5 & 1.0 & 1.0 & 1.0 & 256.90 \\
\hline 0.3 & 0.3 & 0.3 & 0.5 & 0.3 & 0.1 & -0.9 & 0.3 & 0.7 & 1.0 & 1.0 & 1.0 & 288.22 \\
\hline 0.3 & 0.3 & 0.3 & 0.5 & 0.3 & 0.1 & -0.9 & 0.5 & 0.1 & 1.0 & 1.0 & 1.0 & 247.92 \\
\hline 0.3 & 0.3 & 0.3 & 0.5 & 0.3 & 0.1 & -0.9 & 0.5 & 0.3 & 1.0 & 1.0 & 1.0 & 276.96 \\
\hline 0.3 & 0.3 & 0.3 & 0.5 & 0.3 & 0.1 & -0.9 & 0.5 & 0.5 & 1.0 & 1.0 & 1.0 & 313.71 \\
\hline 0.3 & 0.3 & 0.3 & 0.5 & 0.3 & 0.1 & -0.9 & 0.5 & 0.7 & 1.0 & 1.0 & 1.0 & 361.70 \\
\hline 0.3 & 0.3 & 0.3 & 0.5 & 0.3 & 0.1 & -0.9 & 0.7 & 0.1 & 1.0 & 1.0 & 1.0 & 300.42 \\
\hline 0.3 & 0.3 & 0.3 & 0.5 & 0.3 & 0.1 & -0.9 & 0.7 & 0.3 & 1.0 & 1.0 & 1.0 & 344.15 \\
\hline 0.3 & 0.3 & 0.3 & 0.5 & 0.3 & 0.1 & -0.9 & 0.7 & 0.5 & 1.0 & 1.0 & 1.0 & 402.77 \\
\hline 0.3 & 0.3 & 0.3 & 0.5 & 0.3 & 0.1 & -0.9 & 0.7 & 0.7 & 1.0 & 1.0 & 1.0 & 485.46 \\
\hline 0.3 & 0.3 & 0.3 & 0.5 & 0.3 & 0.1 & -0.7 & 0.5 & 0.7 & 1.0 & 1.0 & 1.0 & 204.95 \\
\hline 0.3 & 0.3 & 0.3 & 0.5 & 0.3 & 0.1 & -0.7 & 0.7 & 0.5 & 1.0 & 1.0 & 1.0 & 217.52 \\
\hline 0.3 & 0.3 & 0.3 & 0.5 & 0.3 & 0.1 & -0.7 & 0.7 & 0.7 & 1.0 & 1.0 & 1.0 & 239.55 \\
\hline 0.3 & 0.3 & 0.3 & 0.5 & 0.3 & 0.3 & -0.9 & 0.1 & 0.5 & 1.0 & 1.0 & 1.0 & 213.97 \\
\hline 0.3 & 0.3 & 0.3 & 0.5 & 0.3 & 0.3 & -0.9 & 0.1 & 0.7 & 1.0 & 1.0 & 1.0 & 293.71 \\
\hline 0.3 & 0.3 & 0.3 & 0.5 & 0.3 & 0.3 & -0.9 & 0.3 & 0.3 & 1.0 & 1.0 & 1.0 & 261.26 \\
\hline 0.3 & 0.3 & 0.3 & 0.5 & 0.3 & 0.3 & -0.9 & 0.3 & 0.5 & 1.0 & 1.0 & 1.0 & 390.79 \\
\hline 0.3 & 0.3 & 0.3 & 0.5 & 0.3 & 0.3 & -0.9 & 0.5 & 0.1 & 1.0 & 1.0 & 1.0 & 335.36 \\
\hline 0.3 & 0.3 & 0.3 & 0.5 & 0.3 & 0.3 & -0.7 & 0.3 & 0.5 & 1.0 & 1.0 & 1.0 & 213.97 \\
\hline 0.3 & 0.3 & 0.3 & 0.5 & 0.3 & 0.3 & -0.7 & 0.3 & 0.7 & 1.0 & 1.0 & 1.0 & 293.71 \\
\hline 0.3 & 0.3 & 0.3 & 0.5 & 0.3 & 0.3 & -0.7 & 0.5 & 0.3 & 1.0 & 1.0 & 1.0 & 261.26 \\
\hline 0.3 & 0.3 & 0.3 & 0.5 & 0.3 & 0.3 & -0.7 & 0.5 & 0.5 & 1.0 & 1.0 & 1.0 & 390.79 \\
\hline 0.3 & 0.3 & 0.3 & 0.5 & 0.3 & 0.3 & -0.7 & 0.7 & 0.1 & 1.0 & 1.0 & 1.0 & 335.36 \\
\hline 0.3 & 0.3 & 0.3 & 0.5 & 0.3 & 0.3 & -0.5 & 0.5 & 0.5 & 1.0 & 1.0 & 1.0 & 213.97 \\
\hline 0.3 & 0.3 & 0.3 & 0.5 & 0.3 & 0.3 & -0.5 & 0.5 & 0.7 & 1.0 & 1.0 & 1.0 & 293.71 \\
\hline 0.3 & 0.3 & 0.3 & 0.5 & 0.3 & 0.3 & -0.5 & 0.7 & 0.3 & 1.0 & 1.0 & 1.0 & 261.26 \\
\hline 0.3 & 0.3 & 0.3 & 0.5 & 0.3 & 0.3 & -0.5 & 0.7 & 0.5 & 1.0 & 1.0 & 1.0 & 390.79 \\
\hline 0.3 & 0.3 & 0.3 & 0.5 & 0.3 & 0.3 & -0.3 & 0.7 & 0.5 & 1.0 & 1.0 & 1.0 & 213.97 \\
\hline 0.3 & 0.3 & 0.3 & 0.5 & 0.3 & 0.3 & -0.3 & 0.7 & 0.7 & 1.0 & 1.0 & 1.0 & 293.71 \\
\hline 0.3 & 0.3 & 0.3 & 0.5 & 0.3 & 0.5 & -0.9 & 0.3 & 0.5 & 1.0 & 1.0 & 1.0 & 242.67 \\
\hline 0.3 & 0.3 & 0.3 & 0.5 & 0.3 & 0.5 & -0.9 & 0.3 & 0.7 & 1.0 & 1.0 & 1.0 & 498.42 \\
\hline 0.3 & 0.3 & 0.3 & 0.5 & 0.3 & 0.5 & -0.9 & 0.5 & 0.1 & 1.0 & 1.0 & 1.0 & 207.22 \\
\hline 0.3 & 0.3 & 0.3 & 0.5 & 0.3 & 0.5 & -0.9 & 0.5 & 0.3 & 1.0 & 1.0 & 1.0 & 368.84 \\
\hline
\end{tabular}


IMPROVING THE BAR-LEV, BOBOVITCH \& BOUKAI RANDOM RESPONSE MODELS

Appendix

Table 3.1 (continued): Relative efficiency of the proposed ratio estimator with respect to the BBB model for different choice of parameters with $P=P_{1}=P_{2}=0.7$

\begin{tabular}{|c|c|c|c|c|c|c|c|c|c|c|c|c|}
\hline$C_{\gamma}$ & $C_{\gamma_{1}}$ & $C_{\gamma_{2}}$ & $C_{y}$ & $C x_{1}$ & $C x_{2}$ & $\rho_{y x_{1}}$ & $\rho_{y x_{2}}$ & $\rho_{x_{1} x_{2}}$ & $\theta$ & $\theta_{1}$ & $\theta_{2}$ & $\mathrm{RE}$ \\
\hline 0.3 & 0.3 & 0.3 & 0.5 & 0.3 & 0.5 & -0.7 & 0.3 & 0.7 & 1.0 & 1.0 & 1.0 & 242.67 \\
\hline 0.3 & 0.3 & 0.3 & 0.5 & 0.3 & 0.5 & -0.7 & 0.5 & 0.3 & 1.0 & 1.0 & 1.0 & 207.22 \\
\hline 0.3 & 0.3 & 0.3 & 0.5 & 0.3 & 0.5 & -0.7 & 0.5 & 0.5 & 1.0 & 1.0 & 1.0 & 368.84 \\
\hline 0.3 & 0.3 & 0.3 & 0.5 & 0.3 & 0.5 & -0.7 & 0.7 & 0.1 & 1.0 & 1.0 & 1.0 & 292.74 \\
\hline 0.3 & 0.3 & 0.3 & 0.5 & 0.3 & 0.5 & -0.5 & 0.5 & 0.5 & 1.0 & 1.0 & 1.0 & 207.22 \\
\hline 0.3 & 0.3 & 0.3 & 0.5 & 0.3 & 0.5 & -0.5 & 0.5 & 0.7 & 1.0 & 1.0 & 1.0 & 368.84 \\
\hline 0.3 & 0.3 & 0.3 & 0.5 & 0.3 & 0.5 & -0.5 & 0.7 & 0.3 & 1.0 & 1.0 & 1.0 & 292.74 \\
\hline 0.3 & 0.3 & 0.3 & 0.5 & 0.3 & 0.5 & -0.3 & 0.5 & 0.7 & 1.0 & 1.0 & 1.0 & 207.22 \\
\hline 0.3 & 0.3 & 0.3 & 0.5 & 0.3 & 0.5 & -0.3 & 0.7 & 0.5 & 1.0 & 1.0 & 1.0 & 292.74 \\
\hline 0.3 & 0.3 & 0.3 & 0.5 & 0.3 & 0.5 & -0.1 & 0.7 & 0.7 & 1.0 & 1.0 & 1.0 & 292.74 \\
\hline 0.3 & 0.3 & 0.3 & 0.5 & 0.5 & 0.1 & -0.9 & 0.1 & 0.1 & 1.0 & 1.0 & 1.0 & 210.54 \\
\hline 0.3 & 0.3 & 0.3 & 0.5 & 0.5 & 0.1 & -0.9 & 0.1 & 0.3 & 1.0 & 1.0 & 1.0 & 247.23 \\
\hline 0.3 & 0.3 & 0.3 & 0.5 & 0.5 & 0.1 & -0.9 & 0.1 & 0.5 & 1.0 & 1.0 & 1.0 & 299.41 \\
\hline 0.3 & 0.3 & 0.3 & 0.5 & 0.5 & 0.1 & -0.9 & 0.1 & 0.7 & 1.0 & 1.0 & 1.0 & 379.50 \\
\hline 0.3 & 0.3 & 0.3 & 0.5 & 0.5 & 0.1 & -0.9 & 0.3 & 0.1 & 1.0 & 1.0 & 1.0 & 247.23 \\
\hline 0.3 & 0.3 & 0.3 & 0.5 & 0.5 & 0.1 & -0.9 & 0.3 & 0.3 & 1.0 & 1.0 & 1.0 & 299.41 \\
\hline 0.3 & 0.3 & 0.3 & 0.5 & 0.5 & 0.1 & -0.9 & 0.3 & 0.5 & 1.0 & 1.0 & 1.0 & 379.50 \\
\hline 0.3 & 0.3 & 0.3 & 0.5 & 0.5 & 0.1 & -0.9 & 0.5 & 0.1 & 1.0 & 1.0 & 1.0 & 299.41 \\
\hline 0.3 & 0.3 & 0.3 & 0.5 & 0.5 & 0.1 & -0.9 & 0.5 & 0.3 & 1.0 & 1.0 & 1.0 & 379.50 \\
\hline 0.3 & 0.3 & 0.3 & 0.5 & 0.5 & 0.1 & -0.9 & 0.7 & 0.1 & 1.0 & 1.0 & 1.0 & 379.50 \\
\hline 0.3 & 0.3 & 0.3 & 0.5 & 0.5 & 0.1 & -0.7 & 0.5 & 0.7 & 1.0 & 1.0 & 1.0 & 210.54 \\
\hline 0.3 & 0.3 & 0.3 & 0.5 & 0.5 & 0.1 & -0.7 & 0.7 & 0.5 & 1.0 & 1.0 & 1.0 & 210.54 \\
\hline 0.3 & 0.3 & 0.3 & 0.5 & 0.5 & 0.1 & -0.7 & 0.7 & 0.7 & 1.0 & 1.0 & 1.0 & 247.23 \\
\hline 0.3 & 0.3 & 0.3 & 0.5 & 0.5 & 0.3 & -0.9 & 0.1 & 0.3 & 1.0 & 1.0 & 1.0 & 242.67 \\
\hline 0.3 & 0.3 & 0.3 & 0.5 & 0.5 & 0.3 & -0.9 & 0.1 & 0.5 & 1.0 & 1.0 & 1.0 & 498.42 \\
\hline 0.3 & 0.3 & 0.3 & 0.5 & 0.5 & 0.3 & -0.9 & 0.3 & 0.1 & 1.0 & 1.0 & 1.0 & 242.67 \\
\hline 0.3 & 0.3 & 0.3 & 0.5 & 0.5 & 0.3 & -0.9 & 0.3 & 0.3 & 1.0 & 1.0 & 1.0 & 498.42 \\
\hline 0.3 & 0.3 & 0.3 & 0.5 & 0.5 & 0.3 & -0.9 & 0.5 & 0.1 & 1.0 & 1.0 & 1.0 & 498.42 \\
\hline 0.3 & 0.3 & 0.3 & 0.5 & 0.5 & 0.3 & -0.7 & 0.1 & 0.7 & 1.0 & 1.0 & 1.0 & 292.74 \\
\hline 0.3 & 0.3 & 0.3 & 0.5 & 0.5 & 0.3 & -0.7 & 0.3 & 0.5 & 1.0 & 1.0 & 1.0 & 292.74 \\
\hline 0.3 & 0.3 & 0.3 & 0.5 & 0.5 & 0.3 & -0.7 & 0.5 & 0.3 & 1.0 & 1.0 & 1.0 & 292.74 \\
\hline 0.3 & 0.3 & 0.3 & 0.5 & 0.5 & 0.3 & -0.7 & 0.7 & 0.1 & 1.0 & 1.0 & 1.0 & 292.74 \\
\hline 0.3 & 0.3 & 0.3 & 0.5 & 0.5 & 0.3 & -0.5 & 0.3 & 0.7 & 1.0 & 1.0 & 1.0 & 207.22 \\
\hline 0.3 & 0.3 & 0.3 & 0.5 & 0.5 & 0.3 & -0.5 & 0.5 & 0.5 & 1.0 & 1.0 & 1.0 & 207.22 \\
\hline 0.3 & 0.3 & 0.3 & 0.5 & 0.5 & 0.3 & -0.5 & 0.5 & 0.7 & 1.0 & 1.0 & 1.0 & 368.84 \\
\hline 0.3 & 0.3 & 0.3 & 0.5 & 0.5 & 0.3 & -0.5 & 0.7 & 0.3 & 1.0 & 1.0 & 1.0 & 207.22 \\
\hline 0.3 & 0.3 & 0.3 & 0.5 & 0.5 & 0.3 & -0.5 & 0.7 & 0.5 & 1.0 & 1.0 & 1.0 & 368.84 \\
\hline 0.3 & 0.3 & 0.3 & 0.5 & 0.5 & 0.3 & -0.3 & 0.7 & 0.7 & 1.0 & 1.0 & 1.0 & 242.67 \\
\hline 0.3 & 0.3 & 0.3 & 0.5 & 0.5 & 0.5 & -0.9 & 0.1 & 0.5 & 1.0 & 1.0 & 1.0 & 280.25 \\
\hline 0.3 & 0.3 & 0.3 & 0.5 & 0.5 & 0.5 & -0.9 & 0.3 & 0.3 & 1.0 & 1.0 & 1.0 & 280.25 \\
\hline 0.3 & 0.3 & 0.3 & 0.5 & 0.5 & 0.5 & -0.9 & 0.5 & 0.1 & 1.0 & 1.0 & 1.0 & 280.25 \\
\hline
\end{tabular}




\section{ODUMADE \& SINGH}

Appendix

Table 3.1 (continued): Relative efficiency of the proposed ratio estimator with respect to the BBB model for different choice of parameters with $P=P_{1}=P_{2}=0.7$

\begin{tabular}{|c|c|c|c|c|c|c|c|c|c|c|c|c|}
\hline$C_{\gamma}$ & $C_{\gamma_{1}}$ & $C_{\gamma_{2}}$ & $C_{y}$ & $C x_{1}$ & $C x_{2}$ & $\rho_{y x_{1}}$ & $\rho_{y x_{2}}$ & $\rho_{x_{1} x_{2}}$ & $\theta$ & $\theta_{1}$ & $\theta_{2}$ & $\mathrm{RE}$ \\
\hline 0.3 & 0.3 & 0.3 & 0.5 & 0.5 & 0.5 & -0.7 & 0.1 & 0.7 & 1.0 & 1.0 & 1.0 & 280.25 \\
\hline 0.3 & 0.3 & 0.3 & 0.5 & 0.5 & 0.5 & -0.7 & 0.3 & 0.5 & 1.0 & 1.0 & 1.0 & 280.25 \\
\hline 0.3 & 0.3 & 0.3 & 0.5 & 0.5 & 0.5 & -0.7 & 0.5 & 0.3 & 1.0 & 1.0 & 1.0 & 280.25 \\
\hline 0.3 & 0.3 & 0.3 & 0.5 & 0.5 & 0.5 & -0.7 & 0.7 & 0.1 & 1.0 & 1.0 & 1.0 & 280.25 \\
\hline 0.3 & 0.3 & 0.3 & 0.5 & 0.5 & 0.5 & -0.5 & 0.3 & 0.7 & 1.0 & 1.0 & 1.0 & 280.25 \\
\hline 0.3 & 0.3 & 0.3 & 0.5 & 0.5 & 0.5 & -0.5 & 0.5 & 0.5 & 1.0 & 1.0 & 1.0 & 280.25 \\
\hline 0.3 & 0.3 & 0.3 & 0.5 & 0.5 & 0.5 & -0.5 & 0.7 & 0.3 & 1.0 & 1.0 & 1.0 & 280.25 \\
\hline 0.3 & 0.3 & 0.3 & 0.5 & 0.5 & 0.5 & -0.3 & 0.5 & 0.7 & 1.0 & 1.0 & 1.0 & 280.25 \\
\hline 0.3 & 0.3 & 0.3 & 0.5 & 0.5 & 0.5 & -0.3 & 0.7 & 0.5 & 1.0 & 1.0 & 1.0 & 280.25 \\
\hline 0.3 & 0.3 & 0.3 & 0.5 & 0.5 & 0.5 & -0.1 & 0.7 & 0.7 & 1.0 & 1.0 & 1.0 & 280.25 \\
\hline 0.5 & 0.5 & 0.5 & 0.3 & 0.5 & 0.3 & -0.9 & 0.7 & 0.7 & 1.0 & 1.0 & 1.0 & 211.38 \\
\hline 0.5 & 0.5 & 0.5 & 0.5 & 0.3 & 0.3 & -0.9 & 0.5 & 0.7 & 1.0 & 1.0 & 1.0 & 243.36 \\
\hline 0.5 & 0.5 & 0.5 & 0.5 & 0.3 & 0.3 & -0.9 & 0.7 & 0.3 & 1.0 & 1.0 & 1.0 & 224.31 \\
\hline 0.5 & 0.5 & 0.5 & 0.5 & 0.3 & 0.3 & -0.9 & 0.7 & 0.5 & 1.0 & 1.0 & 1.0 & 293.18 \\
\hline 0.5 & 0.5 & 0.5 & 0.5 & 0.3 & 0.3 & -0.9 & 0.7 & 0.7 & 1.0 & 1.0 & 1.0 & 423.08 \\
\hline 0.5 & 0.5 & 0.5 & 0.5 & 0.3 & 0.3 & -0.7 & 0.7 & 0.7 & 1.0 & 1.0 & 1.0 & 243.36 \\
\hline 0.5 & 0.5 & 0.5 & 0.5 & 0.3 & 0.5 & -0.9 & 0.5 & 0.7 & 1.0 & 1.0 & 1.0 & 265.96 \\
\hline 0.5 & 0.5 & 0.5 & 0.5 & 0.3 & 0.5 & -0.9 & 0.7 & 0.3 & 1.0 & 1.0 & 1.0 & 230.32 \\
\hline 0.5 & 0.5 & 0.5 & 0.5 & 0.3 & 0.5 & -0.9 & 0.7 & 0.5 & 1.0 & 1.0 & 1.0 & 385.15 \\
\hline 0.5 & 0.5 & 0.5 & 0.5 & 0.3 & 0.5 & -0.7 & 0.7 & 0.5 & 1.0 & 1.0 & 1.0 & 230.32 \\
\hline 0.5 & 0.5 & 0.5 & 0.5 & 0.3 & 0.5 & -0.7 & 0.7 & 0.7 & 1.0 & 1.0 & 1.0 & 385.15 \\
\hline 0.5 & 0.5 & 0.5 & 0.5 & 0.3 & 0.5 & -0.5 & 0.7 & 0.7 & 1.0 & 1.0 & 1.0 & 230.32 \\
\hline 0.5 & 0.5 & 0.5 & 0.5 & 0.5 & 0.3 & -0.9 & 0.1 & 0.7 & 1.0 & 1.0 & 1.0 & 203.10 \\
\hline 0.5 & 0.5 & 0.5 & 0.5 & 0.5 & 0.3 & -0.9 & 0.3 & 0.5 & 1.0 & 1.0 & 1.0 & 203.10 \\
\hline 0.5 & 0.5 & 0.5 & 0.5 & 0.5 & 0.3 & -0.9 & 0.3 & 0.7 & 1.0 & 1.0 & 1.0 & 314.65 \\
\hline 0.5 & 0.5 & 0.5 & 0.5 & 0.5 & 0.3 & -0.9 & 0.5 & 0.3 & 1.0 & 1.0 & 1.0 & 203.10 \\
\hline 0.5 & 0.5 & 0.5 & 0.5 & 0.5 & 0.3 & -0.9 & 0.5 & 0.5 & 1.0 & 1.0 & 1.0 & 314.65 \\
\hline 0.5 & 0.5 & 0.5 & 0.5 & 0.5 & 0.3 & -0.9 & 0.7 & 0.1 & 1.0 & 1.0 & 1.0 & 203.10 \\
\hline 0.5 & 0.5 & 0.5 & 0.5 & 0.5 & 0.3 & -0.9 & 0.7 & 0.3 & 1.0 & 1.0 & 1.0 & 314.65 \\
\hline 0.5 & 0.5 & 0.5 & 0.5 & 0.5 & 0.3 & -0.7 & 0.5 & 0.7 & 1.0 & 1.0 & 1.0 & 230.32 \\
\hline 0.5 & 0.5 & 0.5 & 0.5 & 0.5 & 0.3 & -0.7 & 0.7 & 0.5 & 1.0 & 1.0 & 1.0 & 230.32 \\
\hline 0.5 & 0.5 & 0.5 & 0.5 & 0.5 & 0.3 & -0.7 & 0.7 & 0.7 & 1.0 & 1.0 & 1.0 & 385.15 \\
\hline 0.5 & 0.5 & 0.5 & 0.5 & 0.5 & 0.5 & -0.9 & 0.3 & 0.7 & 1.0 & 1.0 & 1.0 & 423.08 \\
\hline 0.5 & 0.5 & 0.5 & 0.5 & 0.5 & 0.5 & -0.9 & 0.5 & 0.5 & 1.0 & 1.0 & 1.0 & 423.08 \\
\hline 0.5 & 0.5 & 0.5 & 0.5 & 0.5 & 0.5 & -0.9 & 0.7 & 0.3 & 1.0 & 1.0 & 1.0 & 423.08 \\
\hline 0.5 & 0.5 & 0.5 & 0.5 & 0.5 & 0.5 & -0.7 & 0.5 & 0.7 & 1.0 & 1.0 & 1.0 & 423.08 \\
\hline 0.5 & 0.5 & 0.5 & 0.5 & 0.5 & 0.5 & -0.7 & 0.7 & 0.5 & 1.0 & 1.0 & 1.0 & 423.08 \\
\hline 0.5 & 0.5 & 0.5 & 0.5 & 0.5 & 0.5 & -0.5 & 0.7 & 0.7 & 1.0 & 1.0 & 1.0 & 423.08 \\
\hline
\end{tabular}


IMPROVING THE BAR-LEV, BOBOVITCH \& BOUKAI RANDOM RESPONSE MODELS

Appendix

Table 4.1: Relative efficiency of the proposed power transformation estimator with respect to the BBB model for different choice of parameters with $P=P_{1}=P_{2}=0.7$

\begin{tabular}{|c|c|c|c|c|c|c|c|c|c|c|c|c|c|}
\hline$C_{\gamma}$ & $C_{\gamma_{1}}$ & $C_{\gamma_{2}}$ & $C_{y}$ & $C x_{1}$ & $C x_{2}$ & $\rho_{y x_{1}}$ & $\rho_{y x_{2}}$ & $\rho_{x_{1} x_{2}}$ & $\theta$ & $\theta_{1}$ & $\theta_{2}$ & $\alpha_{\mathrm{opt}}$ & $\mathrm{RE}$ \\
\hline 0.1 & 0.1 & 0.1 & 0.5 & 0.3 & 0.5 & 0.1 & 0.7 & 0.7 & 0.5 & 0.5 & 0.5 & 1.00 & 221.88 \\
\hline 0.1 & 0.1 & 0.1 & 0.5 & 0.5 & 0.3 & 0.7 & 0.1 & 0.7 & 0.5 & 0.5 & 0.5 & -1.00 & 221.88 \\
\hline 0.1 & 0.1 & 0.1 & 0.5 & 0.5 & 0.5 & 0.1 & 0.7 & 0.7 & 0.5 & 0.5 & 0.5 & 1.04 & 215.47 \\
\hline 0.1 & 0.1 & 0.1 & 0.5 & 0.5 & 0.5 & 0.7 & 0.1 & 0.7 & 0.5 & 0.5 & 0.5 & -1.04 & 215.47 \\
\hline 0.1 & 0.1 & 0.1 & 0.1 & 0.3 & 0.5 & 0.1 & 0.7 & 0.7 & 1.0 & 1.0 & 1.0 & 0.23 & 234.49 \\
\hline 0.1 & 0.1 & 0.1 & 0.1 & 0.5 & 0.3 & 0.7 & 0.1 & 0.7 & 1.0 & 1.0 & 1.0 & -0.23 & 234.49 \\
\hline 0.1 & 0.1 & 0.1 & 0.3 & 0.1 & 0.3 & 0.1 & 0.7 & 0.5 & 1.0 & 1.0 & 1.0 & 0.79 & 202.37 \\
\hline 0.1 & 0.1 & 0.1 & 0.3 & 0.1 & 0.3 & 0.1 & 0.7 & 0.7 & 1.0 & 1.0 & 1.0 & 0.93 & 250.17 \\
\hline 0.1 & 0.1 & 0.1 & 0.3 & 0.1 & 0.5 & 0.1 & 0.7 & 0.5 & 1.0 & 1.0 & 1.0 & 0.47 & 206.00 \\
\hline 0.1 & 0.1 & 0.1 & 0.3 & 0.1 & 0.5 & 0.1 & 0.7 & 0.7 & 1.0 & 1.0 & 1.0 & 0.52 & 230.87 \\
\hline 0.1 & 0.1 & 0.1 & 0.3 & 0.1 & 0.5 & 0.3 & 0.7 & 0.7 & 1.0 & 1.0 & 1.0 & 0.49 & 200.86 \\
\hline 0.1 & 0.1 & 0.1 & 0.3 & 0.3 & 0.1 & 0.7 & 0.1 & 0.5 & 1.0 & 1.0 & 1.0 & -0.79 & 202.37 \\
\hline 0.1 & 0.1 & 0.1 & 0.3 & 0.3 & 0.1 & 0.7 & 0.1 & 0.7 & 1.0 & 1.0 & 1.0 & -0.93 & 250.17 \\
\hline 0.1 & 0.1 & 0.1 & 0.3 & 0.3 & 0.3 & 0.1 & 0.7 & 0.7 & 1.0 & 1.0 & 1.0 & 0.89 & 206.79 \\
\hline 0.1 & 0.1 & 0.1 & 0.3 & 0.3 & 0.3 & 0.7 & 0.1 & 0.7 & 1.0 & 1.0 & 1.0 & -0.89 & 206.79 \\
\hline 0.1 & 0.1 & 0.1 & 0.3 & 0.3 & 0.5 & 0.1 & 0.7 & 0.5 & 1.0 & 1.0 & 1.0 & 0.49 & 200.61 \\
\hline 0.1 & 0.1 & 0.1 & 0.3 & 0.3 & 0.5 & 0.1 & 0.7 & 0.7 & 1.0 & 1.0 & 1.0 & 0.70 & 358.60 \\
\hline 0.1 & 0.1 & 0.1 & 0.3 & 0.5 & 0.1 & 0.7 & 0.1 & 0.5 & 1.0 & 1.0 & 1.0 & -0.47 & 206.00 \\
\hline 0.1 & 0.1 & 0.1 & 0.3 & 0.5 & 0.1 & 0.7 & 0.1 & 0.7 & 1.0 & 1.0 & 1.0 & -0.52 & 230.87 \\
\hline 0.1 & 0.1 & 0.1 & 0.3 & 0.5 & 0.1 & 0.7 & 0.3 & 0.7 & 1.0 & 1.0 & 1.0 & -0.49 & 200.86 \\
\hline 0.1 & 0.1 & 0.1 & 0.3 & 0.5 & 0.3 & 0.7 & 0.1 & 0.5 & 1.0 & 1.0 & 1.0 & -0.49 & 200.61 \\
\hline 0.1 & 0.1 & 0.1 & 0.3 & 0.5 & 0.3 & 0.7 & 0.1 & 0.7 & 1.0 & 1.0 & 1.0 & -0.70 & 358.60 \\
\hline 0.1 & 0.1 & 0.1 & 0.3 & 0.5 & 0.5 & 0.1 & 0.7 & 0.7 & 1.0 & 1.0 & 1.0 & 0.57 & 222.91 \\
\hline 0.1 & 0.1 & 0.1 & 0.3 & 0.5 & 0.5 & 0.7 & 0.1 & 0.7 & 1.0 & 1.0 & 1.0 & -0.57 & 222.91 \\
\hline 0.1 & 0.1 & 0.1 & 0.5 & 0.1 & 0.3 & 0.1 & 0.7 & 0.5 & 1.0 & 1.0 & 1.0 & 1.31 & 206.82 \\
\hline 0.1 & 0.1 & 0.1 & 0.5 & 0.1 & 0.3 & 0.1 & 0.7 & 0.7 & 1.0 & 1.0 & 1.0 & 1.56 & 258.32 \\
\hline 0.1 & 0.1 & 0.1 & 0.5 & 0.1 & 0.5 & 0.1 & 0.7 & 0.5 & 1.0 & 1.0 & 1.0 & 0.78 & 210.69 \\
\hline 0.1 & 0.1 & 0.1 & 0.5 & 0.1 & 0.5 & 0.1 & 0.7 & 0.7 & 1.0 & 1.0 & 1.0 & 0.86 & 237.40 \\
\hline 0.1 & 0.1 & 0.1 & 0.5 & 0.1 & 0.5 & 0.3 & 0.7 & 0.7 & 1.0 & 1.0 & 1.0 & 0.81 & 205.21 \\
\hline 0.1 & 0.1 & 0.1 & 0.5 & 0.3 & 0.1 & 0.7 & 0.1 & 0.5 & 1.0 & 1.0 & 1.0 & -1.31 & 206.82 \\
\hline 0.1 & 0.1 & 0.1 & 0.5 & 0.3 & 0.1 & 0.7 & 0.1 & 0.7 & 1.0 & 1.0 & 1.0 & -1.56 & 258.32 \\
\hline 0.1 & 0.1 & 0.1 & 0.5 & 0.3 & 0.3 & 0.1 & 0.7 & 0.7 & 1.0 & 1.0 & 1.0 & 1.49 & 211.54 \\
\hline 0.1 & 0.1 & 0.1 & 0.5 & 0.3 & 0.3 & 0.7 & 0.1 & 0.7 & 1.0 & 1.0 & 1.0 & -1.49 & 211.54 \\
\hline 0.1 & 0.1 & 0.1 & 0.5 & 0.3 & 0.5 & 0.1 & 0.7 & 0.5 & 1.0 & 1.0 & 1.0 & 0.81 & 204.94 \\
\hline 0.1 & 0.1 & 0.1 & 0.5 & 0.3 & 0.5 & 0.1 & 0.7 & 0.7 & 1.0 & 1.0 & 1.0 & 1.17 & 379.21 \\
\hline 0.1 & 0.1 & 0.1 & 0.5 & 0.5 & 0.1 & 0.7 & 0.1 & 0.5 & 1.0 & 1.0 & 1.0 & -0.78 & 210.69 \\
\hline 0.1 & 0.1 & 0.1 & 0.5 & 0.5 & 0.1 & 0.7 & 0.1 & 0.7 & 1.0 & 1.0 & 1.0 & -0.86 & 237.40 \\
\hline 0.1 & 0.1 & 0.1 & 0.5 & 0.5 & 0.1 & 0.7 & 0.3 & 0.7 & 1.0 & 1.0 & 1.0 & -0.81 & 205.21 \\
\hline 0.1 & 0.1 & 0.1 & 0.5 & 0.5 & 0.3 & 0.7 & 0.1 & 0.5 & 1.0 & 1.0 & 1.0 & -0.81 & 204.94 \\
\hline 0.1 & 0.1 & 0.1 & 0.5 & 0.5 & 0.3 & 0.7 & 0.1 & 0.7 & 1.0 & 1.0 & 1.0 & -1.17 & 379.21 \\
\hline 0.1 & 0.1 & 0.1 & 0.5 & 0.5 & 0.5 & 0.1 & 0.7 & 0.7 & 1.0 & 1.0 & 1.0 & 0.95 & 228.82 \\
\hline 0.1 & 0.1 & 0.1 & 0.5 & 0.5 & 0.5 & 0.7 & 0.1 & 0.7 & 1.0 & 1.0 & 1.0 & -0.95 & 228.82 \\
\hline
\end{tabular}

